\title{
HOMOGENEOUS BOREL SETS OF AMBIGUOUS CLASS TWO
}

\author{
BY
}

FONS Van ENGELEN

\begin{abstract}
We describe and characterize all homogeneous subsets of the Cantor set which are both an $F_{\sigma \delta}$ and a $G_{\delta \sigma}$; it turns out that there are $\omega_{1}$ such spaces.
\end{abstract}

1. Introduction. All spaces under discussion are separable and metrizable. The aim of this paper is to determine and characterize all homogeneous zero-dimensional absolute Borel sets of ambiguous class 2, i.e., all homogeneous Borel sets in the Cantor set $C$ that are both an $F_{\sigma \delta}$ (a countable intersection of $\sigma$-compact sets) and a $G_{\delta \sigma}$ (a countable union of topologically complete spaces). Among the absolute Borel sets of ambiguous class 2 are all spaces that are either $\sigma$-compact or topologically complete (they are the absolute Borel sets of class 1); characterizations of all zero-dimensional homogeneous such spaces have been known since around 1930: in 1910 Brouwer [3] characterized the Cantor set $C$; in 1920 Sierpiński [22] characterized the space of rationals Q; and in 1928 Alexandroff and Urysohn [1] characterized the Cantor set minus a point, $C \backslash\{p\}$, the space of irrationals $\mathbf{P}$, and $\mathbf{Q} \times C$. It is easily seen that, apart from the discrete spaces, these are the only homogeneous zero-dimensional absolute Borel sets of class 1 .

It was not until 1981 that the first homogeneous zero-dimensional absolute Borel set of ambiguous class 2 which is not of class 1 was characterized: in [16] van Mill considered products of $\mathbf{Q}, \mathbf{P}$, and $C$, noted that $\mathbf{Q} \times \mathbf{P}$ was not yet characterized, and filled in this gap; two other elements of this class, $S$ and $T$, which are homogeneous complements of $\mathbf{Q} \times \mathbf{P}$ in the Cantor set, were characterized by van Mill [17] resp., van Douwen ([4]; see also [6]), and $\mathbf{Q} \times S$ and $\mathbf{Q} \times T$ followed in [6] by van Engelen and van Mill.

In this paper we show that there are precisely $\omega_{1}$ homogeneous Borel sets of ambiguous class 2 in $C$; it is unknown how many homogeneous Borel sets (of arbitrary class) there are.

This paper is organized as follows: in $\S 3$ we define topological properties $\mathscr{P}_{\alpha}$, for $\alpha<\omega_{1}$, and describe how, with each of those properties, a finite number of homogeneous Borel sets of ambiguous class 2 in the Cantor set can be associated. $\S \S 4$ and 5 provide us with the lemmas that are fundamental to the construction (for the results of $\$ 5$ we heavily rely on a technique due to Saint-Raymond [21]); the actual construction is carried out in $\$ 6$ (spaces associated with property $\mathscr{P}_{\alpha}$ for some $\alpha<\omega$ ) and $\S 7$ (spaces associated with property $\mathscr{P}_{\alpha}$ for some $\alpha \geqslant \omega$ ). In $\S 8$ we show that $C$ contains no other homogeneous Borel sets of ambiguous class 2 than those of $\S \S 6$ and 7. Finally, $\$ 9$ lists some open questions.

Received by the editors January 3, 1984 and, in revised form, August 13, 1984.

1980 Mathematics Subject Classification. Primary 54H05, 54E35, 54F65; Secondary 03E15.

(C)1985 American Mathematical Society $0002-9947 / 85 \$ 1.00+\$ .25$ per page 
2. Preliminaries. For all undefined terms and notation see Engelking [7] or Kuratowski [15]. $A \approx B$ means that $A$ and $B$ are homeomorphic. Cardinals are initial ordinals, and an ordinal is the set of its predecessors; we put $\mathbf{N}=\omega \backslash\{0\}$ and write $\lim (\beta)$ if $\beta$ is a limit ordinal. All metrics in this paper are denoted by $d$ and assumed to be bounded by 1 ; the diameter of a set $A$ is denoted by $\operatorname{diam}(A)$. A subset of a space $X$ is clopen if it is both closed and open in $X$. A space $X$ is homogeneous if for each $x, y \in X$, there exists a homeomorphism $h: X \rightarrow X$ such that $h(x)=y$; homogeneous with respect to dense copies of $A$ if, for all dense subspaces $A_{1}, A_{2}$ of $X$ such that $A_{1} \approx A \approx A_{2}$, there exists a homeomorphism $h: X \rightarrow X$ such that $h\left[A_{1}\right]=$ $A_{2}$; strongly homogeneous if $U \approx X$ for each nonempty clopen subset $U$ of $X$. It is easily seen that a strongly homogeneous zero-dimensional space is homogeneous. By a complete space we mean a topologically complete (separable metric) space, i.e., a (separable) completely metrizable space, i.e., an absolute $G_{\delta}$. If $\mathscr{P}_{1}$ and $\mathscr{P}_{2}$ are topological properties, we write " $X$ is $\mathscr{P}_{1} \cup \mathscr{P}_{2}$ " if $X=A \cup B$, where $A$ has property $\mathscr{P}_{1}$ and $B$ has property $\mathscr{P}_{2}$.

2.1. Definition. Let $\mathscr{P}$ be a topological property.

(a) $A$ space $X$ is strongly $\sigma-\mathscr{P}$ if $X=\bigcup_{i=1}^{\infty} X_{i}$, where each $X_{i}$ is a closed subspace of $X$ which has property $\mathscr{P}$.

(b) $\mathscr{P}$ is strongly $\sigma$-additive if a space is $\mathscr{P}$ whenever it is strongly $\sigma-\mathscr{P}$.

2.2. Definition. For $n \in \mathbf{N}$ a space $X$ is $\Sigma_{n}$ if $X=\bigcup_{i=1}^{n} X_{i}$, where each $X_{i}$ is strongly $\sigma$-complete; $X$ is $\Sigma_{0}$ if $X=\varnothing$.

2.3. Proposition. Let $X$ be compact and $A \subset X$. The following are equivalent:

(i) $A$ is strongly $\sigma$-complete;

(ii) $X \backslash A=F \cup G$, where $F$ is a $\sigma$-compact and $G$ is a complete subspace of $X$;

(iii) $A=F \cap G$, where $F$ is a $\sigma$-compact and $G$ is a complete subspace of $X$;

(iv) $A=G_{1} \backslash G_{2}$, where $G_{1}$ and $G_{2}$ are complete subspaces of $X$.

Proof. The equivalence of (ii)-(iv) is an immediate consequence of the fact that a subspace of a compact space is complete if and only if its complement is $\sigma$-compact. So assume (i) holds, say $A=\bigcup_{i=1}^{\infty} A_{i}$, where each $A_{i}$ is closed in $A$ and complete. Then

$$
X \backslash A=\bigcup_{i=1}^{\infty}\left(\overline{A_{i}} \backslash A_{i}\right) \cup X \backslash \bigcup_{i=1}^{\infty} \overline{A_{i}} .
$$

Now $\overline{A_{i}} \backslash A_{i}$ is $\sigma$-compact, hence, so is $\bigcup_{i=1}^{\infty}\left(\overline{A_{i}} \backslash A_{i}\right)=F$; and $G=X \backslash \bigcup_{i=1}^{\infty} \overline{A_{i}}$ is complete; so (ii) holds. Conversely, suppose (iii) holds, with $F=\bigcup_{i=1}^{\infty} F_{i}$; it is easily seen that $F_{i} \cap G$ is complete and closed in $A$, so $A=\cup_{i=1}^{\infty}\left(F_{i} \cap G\right)$ is strongly $\sigma$-complete.

We now give topological characterizations of some Borel subsets of the Cantor set; parts (e)-(i) of the next theorem will be reproved in this paper. Here, if $\mathscr{P}$ is a topological property, then a space is nowhere $\mathscr{P}$ if none of its nonempty open subsets is $\mathscr{P}$; note that if $\mathscr{P}$ is a closed-hereditary property, then a zero-dimensional space is nowhere $\mathscr{P}$ if none of its nonempty (basic) clopen subsets is $\mathscr{P}$. Of course, "unique" is "unique up to homeomorphism". 
2.4. Theorem (a) (Brouwer [3]). The Cantor set $C$ is the unique zero-dimensional compact space without isolated points.

(b) (AleXANDroff AND URYSOHN [1]; HausdorfF [12]). The set of irrationals $\mathbf{P}$ is the unique, zero-dimensional, complete, nowhere locally compact space.

(c) (SIERPINSKI [22]). The set of rationals $\mathbf{Q}$ is the unique countable space without isolated points.

(d) (AleXANDroff AND URysohn [1]); VAN Mill [16]. $\mathbf{Q} \times C$ is the unique zero-dimensional, $\sigma$-compact, nowhere countable, nowhere locally compact space.

(e) (VAN MILL [16]). $\mathbf{Q} \times \mathbf{P}$ is the unique, zero-dimensional, strongly $\sigma$-complete, nowhere o-compact, nowhere complete space.

(f) (VAN DouWEN [4]; VAN ENGELEN AND VAN Mill [6]). There exists exactly one zero-dimensional space $T$ which is the union of a complete subspace and a countable subspace and is nowhere $\sigma$-compact and nowhere complete.

(g) (VAN MILL [17]). There exists exactly one zero-dimensional space $S$ which is the union of a complete subspace and a $\sigma$-compact subspace and is nowhere $\sigma$-compact and nowhere the union of a complete subspace and a countable subspace.

(h) (VAN ENGELEN AND VAN MILL [6]). $\mathbf{Q} \times T$ is the unique zero-dimensional space which is the union of a strongly o-complete subspace and a countable subspace and nowhere the union of a complete subspace and a countable subspace and nowhere strongly $\sigma$-complete.

(i) (VAN ENGELEN AND VAN Mill [6]). $\mathbf{Q} \times S$ is the unique zero-dimensional space which is the union of a strongly o-complete subspace and a o-compact subspace and nowhere the union of a complete subspace and a $\sigma$-compact subspace and nowhere the union of a strongly $\sigma$-complete subspace and a countable subspace.

A slightly different version of the following lemma was proved in [6].

2.5. Lemma. Let $A$ be a nonempty, compact, nowhere dense subset of $X$, and let $\left(\varepsilon_{n}\right)_{n \in N}$ be a given sequence of positive numbers. Then there exists a countable discrete subset $D=\left\{d_{n}: n \in \mathbf{N}\right\}$ of $X$ such that $\bar{D}=D \cup A$ and $d\left(d_{n}, A\right)<\varepsilon_{n}$ for each $n \in \mathbf{N}$.

Proof. For each $i \in \mathbf{N}$ let $\mathscr{D}_{i}=\left\{D(i, j): j=1, \ldots, n_{i}\right\}$ be a collection of open subsets of $X$ of diameter less than $1 / i$ such that $A \subset \cup \mathscr{D}_{i}$, and let $p(i, j) \in D(i, j)$ $\cap A$. For each $n \in \mathbf{N}$ there exists a unique $i \in \mathbf{N}$ such that $n=\left(\sum_{k=1}^{i-1} n_{k}\right)+j$ for some $j \leqslant n_{i}$, and we choose $d_{n} \in\left(B\left(p(i, j), \varepsilon_{n}\right) \cap D(i, j)\right) \backslash A$. Then $D=\left\{d_{n}\right.$ : $n \in \mathbf{N}\}$ is as required.

3. A preview. The aim of this section is to give the reader a rough idea, without going into technical details, of how the collection of homogeneous Borel sets of ambiguous class 2 in the Cantor set is built up. The topological properties characterizing these spaces will be defined inductively; the definitions are closely related to an old theorem of Kuratowski.

3.1. Definition. For $n \in \omega, \mathscr{K}_{2 n}$ is the class of all spaces which are $\Sigma_{n}$, and for even $\alpha \in\left[\omega, \omega_{1}\right)$, say $\alpha=\beta+2 n$ with $\lim (\beta)$ and $n \in \omega, \mathscr{K}_{\alpha}$ is the class of all spaces $A$ which can be written as $\bigcup_{i=1}^{\infty} A_{i} \cup B$, where $A_{i} \in \cup\left\{\mathscr{K}_{\gamma}: \gamma\right.$ even $\left.<\beta\right\}$ is closed in $A$ and $B$ is $\Sigma_{n}$. 
3.2. TheOREM (KURATOWSKI [15]; FOR $\alpha=0$, HAUSDORFF [11]). Let $X$ be compact, and let $\alpha<\omega_{1}$. Then $A$ is of ambiguous class $\alpha+1$ in $X$ if and only if there exists, for some even $\gamma<\omega_{1}$, a decreasing sequence $\left\{B_{\beta}: \beta<\gamma\right\}$ of sets of the multiplicative class $\alpha$ in $X$ such that $A=\bigcup\left\{B_{\beta} \backslash B_{\beta+1}: \beta\right.$ even $\left.<\gamma\right\}$.

Of course, here we are interested in the case $\alpha=1$ of the above theorem; the sets $B_{\beta}$ will then be $G_{\delta}$ subsets of $X$. If $A$ is of ambiguous class 2 in $X$, we write $A=\mathscr{S}\left[B_{\beta}, \beta<\gamma\right]$ if $\left\{B_{\beta}: \beta<\gamma\right\}$ is a decreasing sequence of $G_{\delta}$ 's in $X$ such that $A=\bigcup\left\{B_{\beta} \backslash B_{\beta+1}: \beta\right.$ even $\left.<\gamma\right\}$.

\subsection{ThEOREM. If $A=\mathscr{S}\left[B_{\beta}, \beta<\gamma\right]$ for some even $\gamma<\omega_{1}$, then $A \in \mathscr{K}_{\gamma}$.}

Proof. If $\gamma=2 n$ for some $n \in \mathbf{N}$, then $A=\bigcup_{i=0}^{n-1} B_{2 i} \backslash B_{2 i+1}$. By Proposition 2.3 each $B_{2 i} \backslash B_{2 i+1}$ is strongly $\sigma$-complete, so $A$ is $\Sigma_{n}$. Now suppose the theorem has been proved for $\gamma<\alpha=\delta+2 n$, with $\lim (\delta)$ and $n \in \omega$, and suppose $A=$ $\mathscr{S}\left[B_{\beta}, \beta<\alpha\right]$. First note that $A=\mathscr{S}\left[B_{\beta}, \beta<\delta\right] \cup \mathscr{S}\left[B_{\delta+i}, i<2 n\right]$ and $B=$ $\mathscr{S}\left[B_{\delta+i}, i<2 n\right]$ is $\Sigma_{n}$. Since $\delta$ is a limit,

$$
\mathscr{S}\left[B_{\beta}, \beta<\delta\right]=\bigcup\left\{\mathscr{S}\left[B_{\beta}, \beta<\gamma\right]: \gamma \text { even }<\delta\right\},
$$

and $\mathscr{S}\left[B_{\beta}, \beta<\gamma\right]=A \backslash B_{\gamma}$ is an $F_{\sigma}$ in $A$ for each even $\gamma<\delta$; put $A \backslash B_{\gamma}=\bigcup_{i=1}^{\infty} F_{i}^{\gamma}$, with $F_{i}^{\gamma}$ closed in $A$ for each $i \in \mathbf{N}$. Then $\mathscr{S}\left[B_{\beta}, \beta<\delta\right]=$ $\bigcup\left\{F_{i}^{\gamma}: i \in \mathbf{N}, \gamma\right.$ even $\left.<\delta\right\}$ and $F_{i}^{\gamma}=\mathscr{S}\left[F_{i}^{\gamma} \cap B_{\beta}, \beta<\gamma\right] \in \mathscr{K}_{\gamma}$ by the inductive hypothesis.

Thus, every Borel set of ambiguous class 2 belongs to some $\mathscr{K}_{\gamma}$. Conversely, we have

3.4. TheOREM. If $X$ is compact and $A \subset X, A \in \mathscr{K}_{\gamma}$, then $A$ is of ambiguous class 2 in $X$.

Proof. A strongly $\sigma$-complete subspace is the difference of two $G_{\delta}$ 's and, hence, both an $F_{\sigma \delta}$ and a $G_{\delta \sigma}$, so the theorem holds for finite $\gamma$. Now suppose the theorem has been proved for $\gamma<\alpha<\omega_{1}$, say $\alpha=\beta+2 n$ with $\lim (\beta)$ and $n \in \omega$, and suppose $A=\bigcup_{i=1}^{\infty} A_{i} \cup B$ as in Definition 3.1. Then clearly $A$ is a $G_{\delta \sigma}$ by the inductive hypothesis, and

$$
X \backslash A=\bigcup_{i=1}^{\infty}\left(\overline{A_{i}} \backslash A_{i}\right) \cup\left(X \backslash \bigcup_{i=1}^{\infty} \overline{A_{i}}\right) \backslash B .
$$

Since $A_{i}$ is an $F_{\sigma \delta}, \overline{A_{i}} \backslash A_{i}$ is a $G_{\delta \sigma}$; and $\left(X \backslash \bigcup_{i=1}^{\infty} \overline{A_{i}}\right) \backslash B$ is the difference of a $G_{\delta}$ and an $F_{\sigma \delta}$, hence a $G_{\delta \sigma}$; so $X \backslash A$ is a $G_{\delta \sigma}$ as well.

In fact, it is possible to prove the stronger statement that, if $A \in K_{\gamma}$, then $A=\mathscr{S}\left[B_{\beta}, \beta<\gamma\right]$ for some decreasing sequence $\left\{B_{\beta}: \beta<\gamma\right\}$ of $G_{\delta}$ subsets of $X$ (for details, see [5]).

From the above theorems it follows that, in order to characterize Borel sets of ambiguous class 2 , we have to examine the classes $\mathscr{K}_{\gamma}$. Let us first consider the class $\mathscr{K}_{2}$ of spaces which are strongly $\sigma$-complete. This class includes the complete spaces, the $\sigma$-compact spaces, and, hence, in particular, the countable spaces. The next class, $\mathscr{K}_{4}$, contains spaces which are the union of two elements of $\mathscr{K}_{2}$; combining the topological properties mentioned above, we obtain six new properties (note that 
complete $\cup$ complete $\equiv$ complete, countable $\cup$ countable $\equiv$ countable, and $\sigma$-compact $\cup \sigma$-compact $\equiv \sigma$-compact):

complete $\cup$ countable;

strongly $\sigma$-complete $U$ countable;

complete $\cup \sigma$-compact;

strongly $\sigma$-complete $\cup \sigma$-compact;

strongly $\sigma$-complete $\cup$ complete;

strongly $\sigma$-complete $\cup$ strongly $\sigma$-complete.

The class $\mathscr{X}_{6}$ again yields six new topological properties; and proceeding in this way we obtain topological properties describing elements of $\cup_{n \in N} \mathscr{X}_{2 n}$, as follows:

3.5. Definition. Let $X$ be a topological space. Then for each $k \in \omega$,

$X$ has property $\mathscr{P}_{4 k}$ iff $X$ is $\Sigma_{k} \cup$ complete;

$X$ has property $\mathscr{P}_{4 k+1}$ iff $X$ is $\Sigma_{k+1}$;

$X$ has property $\mathscr{P}_{4 k+2}^{1}$ iff $X$ is $\Sigma_{k} \cup$ complete $\cup$ countable;

$X$ has property $\mathscr{P}_{4 k+3}^{1}$ iff $X$ is $\Sigma_{k+1} \cup$ countable (also for $k=-1$ );

$X$ has property $\mathscr{P}_{4 k+2}^{2+}$ iff $X$ is $\Sigma_{k} \cup$ complete $\cup$ o-compact;

$X$ has property $\mathscr{P}_{4 k+3}^{2}$ iff $x$ is $\Sigma_{k+1} \cup \sigma$-compact (also for $k=-1$ ).

For the sake of simplicity we write " $X$ is $\mathscr{P}_{n}^{(i)}$ " if $X$ has one of the properties defined above, meaning that the index $i$ may or may not be there.

Now suppose that the properties $\mathscr{P}_{n}^{(i)}$ are ordered as in the definition, i.e.,

$$
\mathscr{P}_{4 k}<\mathscr{P}_{4 k+1}<\mathscr{P}_{4 k+2}^{1}<\mathscr{P}_{4 k+3}^{1}<\mathscr{P}_{4 k+2}^{2}<\mathscr{P}_{4 k+3}^{2}<\mathscr{P}_{4(k+1)} \text {. }
$$

In $\$ 6$ we show that for each $n \in \omega, i \in\{1,2\}$, there exists, up to homeomorphism, exactly one zero-dimensional space $X_{n}^{(i)}$ which is $\mathscr{P}_{n}^{(i)}$ and nowhere $\mathscr{P}_{m}^{(j)}$ for each $\mathscr{P}_{m}(j)<\mathscr{P}_{n}^{(i)}$.

The spaces $X_{n}^{(i)}$ are roughly described by the following diagram:

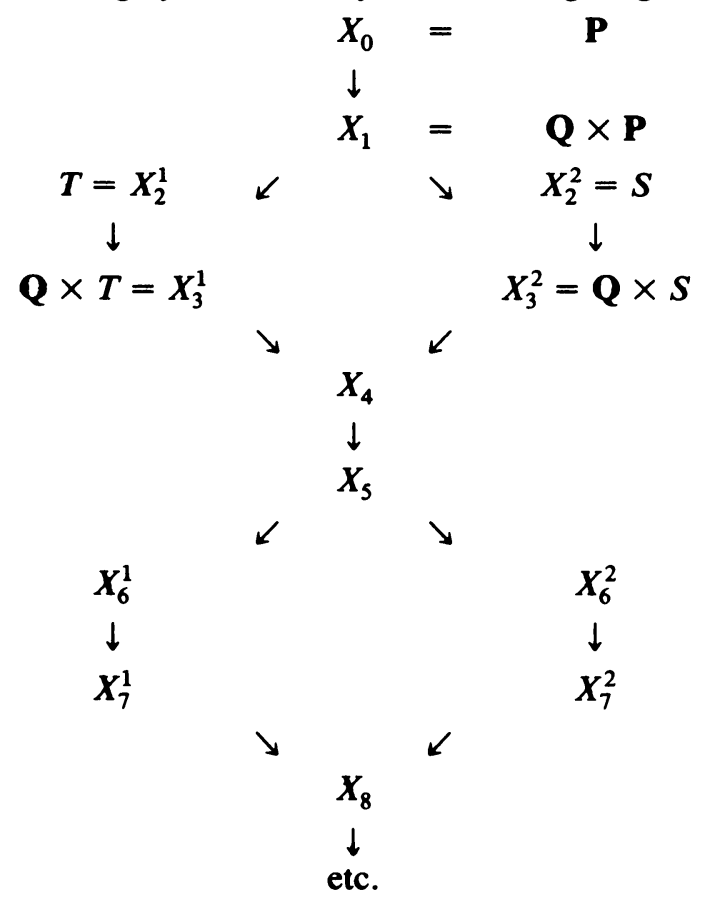


Here, the spaces $S$ and $T$ are those of Theorem 2.4; they are the only homogeneous complements of $\mathbf{Q} \times \mathbf{P}$ in the Cantor set. $X_{4}$ is obtained as the complement of $\mathbf{Q} \times S$ or $\mathbf{Q} \times T$ in the Cantor set, $X_{5}=\mathbf{Q} \times X_{4}$, and $X_{6}^{1}$ and $X_{6}^{2}$ are the only homogeneous complements of $X_{5}$ in the Cantor set. Then $X_{7}^{1}=\mathbf{Q} \times X_{6}^{1}, X_{7}^{2}=\mathbf{Q} \times$ $X_{6}^{2}$, etc.

The spaces described above turn out to be the only zero-dimensional homogeneous (non- $\sigma$-compact) elements of $\bigcup_{n \in \mathrm{N}} \mathscr{K}_{2 n}$. We now turn to the classes $\mathscr{K}_{\alpha+2 n}$, where $n \in \omega$ and $\alpha$ is a limit ordinal less than $\omega_{1}$.

Let us consider the class $\mathscr{K}_{\omega+1}$; each member $X$ of this class is of the form $\bigcup_{i=1}^{\infty} A_{i} \cup B$, where $A_{i}$ is closed in $X, A_{i} \in \bigcup_{n \in \mathrm{N}} \mathscr{K}_{2 n}$, and $B$ is strongly $\sigma$-complete. If $B$ is $\sigma$-compact, say $B=\bigcup_{i=1}^{\infty} B_{i}$ with $B_{i}$ compact, then $X=\bigcup_{i=1}^{\infty}\left(A_{i} \cup B_{i}\right)$, i.e., $X$ would already be in $\mathscr{K}_{\omega}$; the $\sigma$-compact part of $X$ is "absorbed" by $\cup_{i=1}^{\infty} A_{i}$. Hence, if we want to define topological properties as in Definition 3.5, we need only distinguish between " $B$ is complete" and " $B$ is strongly $\sigma$-complete".

3.6. Definition. Let $X$ be a topological space, and let $\alpha \in\left[\omega, \omega_{1}\right)$ be a limit ordinal. Then for each $n \in \omega$,

$X$ has property $\mathscr{P}_{\alpha+2 n}$ iff $X \in \mathscr{K}_{\alpha+2 n}$;

$X$ has property $\mathscr{P}_{\alpha+2 n+1}$ iff $X$ is $\mathscr{P}_{\alpha+2 n} \cup$ complete.

In $\S 7$ we will show that for each $n \in \omega, i \in\{1,2\}$, and $k \in\{0,1\}$, there exists, up to homeomorphism, exactly one zero-dimensional space $X_{\alpha+2 n+k}^{i}$ such that

$X_{\alpha}^{1}=X_{\alpha}^{2}=X_{\alpha}$ is $\mathscr{P}_{\alpha}$, and nowhere $\mathscr{P}_{\beta}$ for each $\beta<\alpha$;

$X_{\alpha+2 n+1}^{1}$ is $\mathscr{P}_{\alpha+2 n+1}$, nowhere $\mathscr{P}_{\alpha+2 n}$, and contains no closed copies of $X_{\alpha+2 n}^{2}$;

$X_{\alpha+2 n+1}^{2}$ is $\mathscr{P}_{\alpha+2 n+1}$, nowhere $\mathscr{P}_{\alpha+2 n}$, and every clopen subset of $X_{\alpha+2 n+1}^{2}$ contains a closed copy of $X_{\alpha+2 n}^{2}$;

$X_{\alpha+2 n+2}^{1}$ is $\mathscr{P}_{\alpha+2 n+2}$, nowhere $\mathscr{P}_{\alpha+2 n+1}$, and contains no closed copies of $X_{\alpha+2 n+1}^{2}$;

$X_{\alpha+2 n+2}^{2}$ is $\mathscr{P}_{\alpha+2 n+2}$, nowhere $\mathscr{P}_{\alpha+2 n+1}$, and every clopen subset of $X_{\alpha+2 n+2}^{2}$ contains a closed copy of $X_{\alpha+2 n+1}^{2}$.

The spaces can be described as follows:

$$
\begin{aligned}
X_{\alpha} & \rightarrow X_{\alpha+1}^{1} \rightarrow X_{\alpha+2}^{1}=\mathbf{Q} \times X_{\alpha+1}^{1} \rightarrow X_{\alpha+1}^{2} \rightarrow X_{\alpha+2}^{2} \\
& =\mathbf{Q} \times X_{\alpha+1}^{2} \rightarrow X_{\alpha+3}^{1} \rightarrow X_{\alpha+4}^{1} \rightarrow X_{\alpha+3}^{2} \rightarrow X_{\alpha+4}^{2} \rightarrow \text { etc. }
\end{aligned}
$$

Here, $X_{\alpha+1}^{1}$ is the complement of $X_{\alpha}$ in the Cantor set, $X_{\alpha+1}^{2}$ is the complement of $X_{\alpha+2}^{1}, X_{\alpha+3}^{1}$ is the complement of $X_{\alpha+2}^{2}$, and so on. Furthermore, $X_{\alpha+4}^{1}=\mathbf{Q} \times X_{\alpha+3}^{1}$, $X_{\alpha+4}^{2}=\mathbf{Q} \times X_{\alpha+3}^{2}$, etc. $X_{\alpha}$ is obtained as the countable union of closed nowhere dense copies of previously defined spaces.

This way we obtain all homogeneous Borel sets of ambiguous class 2 in the Cantor set; this is proved in $\S 8$.

4. KR-covers and their applications. From $\$ 3$ we see that to obtain "new spaces from old", we frequently apply the following operations:

(1) taking the product with $\mathbf{Q}$;

(2) taking complements in the Cantor set.

In this section we establish two very general theorems. The first (Theorem 4.3) is due to Ostrovskii [18] and will enable us to handle (1); since Ostrovskiï's paper is in 
Russian, we feel that it might be useful to include a proof of this theorem. The second (Lemma 4.6) will be used to show that, in all cases under consideration, if we have two spaces $X_{1}$ and $X_{2}$ which have as their complement in the Cantor set the previously characterized space $\mathbf{Q} \times X$, then there exists an autohomeomorphism $h$ of the Cantor set such that $h[\mathbf{Q} \times X]=\mathbf{Q} \times X$, and hence $X_{1} \approx X_{2}$; this will take care of (2).

4.1. Definition. Let $X$ and $Y$ be zero-dimensional spaces, $A$ a closed nowhere dense subset of $X$, and $B$ a closed nowhere dense subset of $Y$. Suppose $h_{0}: A \rightarrow B$ is a homeomorphism, $\mathscr{U}=\left\{\mathscr{U}_{n}: n \in \mathbf{N}\right\}$ is a cover of $X \backslash A$ by disjoint nonempty clopen subsets of $X$, and $\mathscr{V}=\left\{V_{n}: n \in \mathbf{N}\right\}$ is a cover of $Y \backslash B$ by disjoint nonempty clopen subsets of $Y$. Then $\left\{\left(U_{n}, V_{n}\right): n \in \mathbf{N}\right\}$ is a Knaster-Reichbach cover, or KR-cover, for $\left(X \backslash A, Y \backslash B, h_{0}\right)$ if, whenever $h_{n}: U_{n} \rightarrow V_{n}$ is a bijection for each $n \in \mathbf{N}$, the combination mapping $h=\bigcup_{n \in \omega} h_{n}: X \rightarrow Y$ is continuous in points of $A$, and $h^{-1}$ is continuous in points of $B$.

Note that we do not require the bijections $h_{n}: U_{n} \rightarrow V_{n}$ to exist. Objects similar to those defined above were used by Knaster and Reichbach in [14] to prove some theorems on extensions of homeomorphisms; their technique has afterwards been used by various authors, e.g. Pollard [19], Ravdin [20], and Gutek [10].

The following lemma is a slight generalization of Knaster and Reichbach's theorem.

4.2. Lemma. Let $X$ and $Y$ be zero-dimensional spaces, and let $A$ and $B$ be closed nowhere dense subspaces of $X$ and $Y$, respectively. If $h_{0}: A \rightarrow B$ is a homeomorphism, then there exists a $K R$-cover for $\left(X \backslash A, Y \backslash B, h_{0}\right)$.

Proof. Embed $X$ densely in a compact zero-dimensional space $K$. For each $x \in K \backslash \bar{A}$ let $D_{x}$ be clopen such that $\operatorname{diam}\left(D_{x}\right)<d\left(D_{x}, \bar{A}\right)$ and $x \in D_{x}$; let $\left\{D_{i}\right.$ : $i \in \mathbf{N}\}$ be a countable subcover of $\left\{D_{x}: x \in K \backslash \bar{A}\right\}$ and put $\tilde{U}_{i}=D_{i} \backslash\left(\cup_{i<j} D_{j}\right)$. Then $\left\{\tilde{U}_{i}: i \in \mathbf{N}\right\}$ is infinite since $\bar{A}$ is nowhere dense in $K$, and for each $i \in \mathbf{N}$, $\operatorname{diam}\left(\tilde{U}_{i}\right)<d\left(\tilde{U}_{i}, \bar{A}\right)$. Also, since $\{x \in K: d(x, \bar{A}) \geqslant \varepsilon\}$ is compact, $d\left(\tilde{U}_{i}, \bar{A}\right) \rightarrow 0$ $(i \rightarrow \infty)$. Put $U_{i}=\tilde{U}_{i} \cap X$, then $\left\{U_{i}: i \in \mathbf{N}\right\}$ is a cover of $X \backslash A$ by nonempty pairwise disjoint clopen subsets of $X$ satisfying $\operatorname{diam}\left(U_{i}\right)<d\left(U_{i}, A\right) \rightarrow 0(i \rightarrow \infty)$. Similarly, let $\left\{V_{i}: i \in \mathbf{N}\right\}$ be a cover of $Y \backslash B$ by nonempty pairwise disjoint clopen subsets of $Y$ satisfying $\operatorname{diam}\left(V_{i}\right)<d\left(V_{i}, B\right) \rightarrow 0(i \rightarrow \infty)$.

Now define bijections $\rho, \sigma: \mathbf{N} \rightarrow \mathbf{N}$ and points $a_{n} \in A, b_{n}=h_{0}\left(a_{n}\right) \in B$, such that

if $n$ is odd, $d\left(U_{\rho(n)}, a_{n}\right)<2 d\left(U_{\rho(n)}, A\right), V_{\sigma(n)} \subset B\left(b_{n}, d\left(U_{\rho(n)}, A\right)\right)$;

if $n$ is even, $d\left(V_{\sigma(n)}, b_{n}\right)<2 d\left(V_{\sigma(n)}, B\right), U_{\rho(n)} \subset B\left(a_{n}, d\left(V_{\sigma(n)}, B\right)\right)$, as follows:

Suppose $a_{k}, b_{k}, \rho(k), \sigma(k)$ have been defined for $k<n$; if $n$ is odd, let $\rho(n)=\min \mathbf{N} \backslash\{\rho(k): k<n\}$; let $a_{n} \in A$ be such that $d\left(U_{\rho(n)}, a_{n}\right)<2 d\left(U_{\rho(n)}, A\right)$ and let $b_{n}=h_{0}\left(a_{n}\right)$. Put $\varepsilon=\frac{1}{2} d\left(U_{\rho(n)}, A\right)$. Since $d\left(V_{i}, B\right)>0$ for each $i \in \mathbf{N}$ and $B$ is nowhere dense in $Y, B\left(b_{n}, \varepsilon\right) \cap V_{i} \neq \varnothing$ for infinitely many $i \in \mathbf{N}$. Let $\sigma(n) \in$ $\mathbf{N} \backslash\{\sigma(k): k<n\}$ be such that $V_{\sigma(n)} \cap B\left(b_{n}, \varepsilon\right) \neq \varnothing$ and $\operatorname{diam}\left(V_{\sigma(n)}\right)<\varepsilon$. Then $V_{\sigma(n)} \subset B\left(b_{n}, 2 \varepsilon\right)$. 
If $n$ is even let $\sigma(n)=\min \mathbf{N} \backslash\{\sigma(k): k<n\}$ and proceed as above. We claim that $\left\{\left(U_{\rho(n)}, V_{\sigma(n)}\right): n \in \mathbf{N}\right\}$ is a KR-cover for $\left(X \backslash A, Y \backslash B, h_{0}\right)$. Indeed, let $h_{n}$ : $U_{n} \rightarrow V_{n}$ be a bijection, and let $h=\cup_{n \in \omega} h_{n}$. Suppose $a \in A$ and $x_{n} \rightarrow a$. Since $h \mid A$ : $A \approx B$, we may assume that $x_{n} \in U_{\rho\left(i_{n}\right)}$ for each $n \in \mathbf{N}$. Since each $U_{i}$ is clopen in $X$, we have $\rho\left(i_{n}\right) \rightarrow \infty$; hence $\operatorname{diam}\left(U_{\rho\left(i_{n}\right)}\right), d\left(U_{\rho\left(i_{n}\right)}, A\right), \operatorname{diam}\left(V_{\sigma\left(i_{n}\right)}\right), d\left(V_{\sigma\left(i_{n}\right)}, B\right) \rightarrow 0$. Now for each $n \in \mathbf{N}$,

$$
\begin{aligned}
d\left(a_{i_{n}}, a\right) & \leqslant d\left(a_{i_{n}}, x_{n}\right)+d\left(x_{n}, a\right) \leqslant d\left(a_{i_{n}}, U_{\rho\left(i_{n}\right)}\right)+\operatorname{diam}\left(U_{\rho\left(i_{n}\right)}\right)+d\left(x_{n}, a\right) \\
& \leqslant \max \left[d\left(V_{\sigma\left(i_{n}\right)}, B\right), 2 d\left(A, U_{\rho\left(i_{n}\right)}\right)\right]+\operatorname{diam}\left(U_{\rho\left(i_{n}\right)}\right)+d\left(x_{n}, a\right) \rightarrow 0 .
\end{aligned}
$$

So $a_{i_{n}} \rightarrow a$; hence $h\left(a_{i_{n}}\right)=b_{i_{n}} \rightarrow h(a)$. Therefore,

$$
\begin{aligned}
d\left(h\left(x_{n}\right), h(a)\right) & \leqslant d\left(h\left(x_{n}\right), b_{i_{n}}\right)+d\left(b_{i_{n}}, h(a)\right) \\
& \leqslant d\left(b_{i_{n}}, V_{\sigma\left(i_{n}\right)}\right)+\operatorname{diam}\left(V_{\sigma\left(i_{n}\right)}\right)+d\left(b_{i_{n}}, h(a)\right) \\
& \leqslant \max \left[d\left(U_{\rho\left(i_{n}\right)}, A\right), 2 d\left(B, V_{\sigma\left(i_{n}\right)}\right)\right]+\operatorname{diam}\left(V_{\sigma\left(i_{n}\right)}\right)+d\left(b_{i_{n}}, h(a)\right) \\
& \rightarrow 0 .
\end{aligned}
$$

So $h\left(x_{n}\right) \rightarrow h(a)$. Similarly, if $b \in B$ and $y_{n} \rightarrow b$, then $h^{-1}\left(y_{n}\right) \rightarrow h^{-1}(b)$.

4.3. Theorem (Ostrovskil [18]). Let $A$ be a strongly homogeneous, zero-dimensional space and suppose $X=\bigcup_{i=0}^{\infty} X_{i}$, where each $X_{i}$ is closed and nowhere dense in $X$ and $X_{i} \approx A$ for each $i$. Then $X \approx \mathbf{Q} \times A$.

Proof. Let $Y=\mathbf{Q} \times A$; put $\mathbf{Q}=\left\{q_{i}: i \in \omega\right\}$, and let $Y_{i}=\left\{q_{i}\right\} \times A$. Note that for each $i, Y_{i}$ is closed and nowhere dense in $Y$, and $Y_{i} \approx A$. Let $M$ be the set of all finite sequences of natural numbers, including the empty sequence $\varnothing$. For $s=$ $\left(s_{1}, \ldots, s_{k}\right) \in M$, put $|s|=k$, and $|\varnothing|=0$. For each $s \in M$ we define collections $\mathscr{U}(s)=\{U(s, n): n \in \mathbf{N}\}$ of disjoint clopen subsets of $X, \mathscr{V}(s)=\{V(s, n): n \in \mathbf{N}\}$ of disjoint clopen subsets of $Y$, closed nowhere dense subsets $D(s)$ of $U(s), E(s)$ of $V(s)$, and homeomorphisms $h(s): D(s) \rightarrow E(s)$ such that

(1) $D(\varnothing)=X_{0}, E(\varnothing)=Y_{0}, U(\varnothing)=X, V(\varnothing)=Y$;

(2) $\{(U(s, n), V(s, n)): n \in \mathbf{N}\}$ is a $\mathrm{KR}$-cover for $(U(s) \backslash D(s), V(s) \backslash E(s)$, $h(s))$;

(3) $X_{k} \subset \bigcup_{|s| \leqslant k} D(s), Y_{k} \subset \bigcup_{|s| \leqslant k} E(s)$.

Define $D(\varnothing), E(\varnothing), U(\varnothing)$, and $V(\varnothing)$ as in (1); then, by Lemma 4.2, there exists a KR-cover $\{(U(n), V(n)): n \in \mathbf{N}\}$ for $(U(\varnothing) \backslash D(\varnothing), V(\varnothing) \backslash E(\varnothing), h(\varnothing))$, where $h(\varnothing): D(\varnothing) \rightarrow E(\varnothing)$ is an arbitrary homeomorphism.

Now suppose $\mathscr{U}(s), \mathscr{V}(s), D(s), E(s)$, and $h(s)$ have been defined for $|s| \leqslant k$, and fix $s \in M$ with $|s|=k$. Let $k_{n}=\min \left\{j: U(s, n) \cap X_{j} \neq \varnothing\right\}, \quad l_{n}=$ $\min \left\{j: V(s, n) \cap Y_{j} \neq \varnothing\right\}$, and put $D(s, n)=U(s, n) \cap X_{k_{n}}, E(s, n)=V(s, n) \cap$ $Y_{l_{n}}$. Since $A$ is strongly homogeneous, there exists a homeomorphism $h(s, n)$ : $D(s, n) \rightarrow E(s, n)$. Since $D(s, n)$ (resp. $E(s, n)$ ) is closed and nowhere dense in $U(s, n)$ (resp. $V(s, n))$, there exist $\mathscr{U}(s, n)=\{U(s, n, i): i \in \mathbf{N}\}$ and $\mathscr{V}(s, n)=$ $\{V(s, n, i): i \in \mathbf{N}\}$ such that $\{(U(s, n, i), V(s, n, i)): i \in \mathbf{N}\}$ is a KR-cover for $(U(s, n) \backslash D(s, n), V(s, n) \backslash E(s, n), h(s, n))$. It is easily seen that (3) is satisfied. 
This completes the induction. We claim that $h=\bigcup_{s \in M} h_{s}: X \approx Y$. Since $D\left(s_{1}\right) \cap$ $D\left(s_{2}\right)=\varnothing$ if $s_{1} \neq s_{2}, h$ is well defined, and, by (3), the domain of $h$ is all of $X$, and the range is all of $Y$. Since $h$ is clearly bijective, it suffices to show that $h$ and $h^{-1}$ are continuous. So let $x \in X$, say $x \in D(s)$. By (2) it suffices to show that $h[U(s, n)]=$ $V(s, n)$ for each $n \in \mathbf{N}$; but this follows immediately from the observation that $U(s, n)=\bigcup_{t \in M} D(s, n, t), V(s, n)=\bigcup_{t \in M} E(s, n, t)$, and $h[D(s, n, t)]=E(s, n, t)$ for each $t \in M$. The continuity of $h^{-1}$ follows in exactly the same way.

4.4. REMARK. If the strongly homogeneous space $A$ in the above theorem contains a closed nowhere dense copy of itself, then the result can also be deduced from a theorem of van Mill [16].

The following theorem is a slight modification of the convergence criterion of Anderson [2].

4.5. Theorem. Let $X$ be compact, and for each $n \in \mathbf{N}$ let $h_{n}: X \rightarrow X$ be a homeomorphism such that

$$
\begin{aligned}
& d\left(h_{n+1}, h_{n}\right)<\min \left\{2^{-n}, 3^{-n} \cdot \min \left\{\operatorname { m i n } \left\{d\left(h_{i}(x), h_{i}(y)\right):\right.\right.\right. \\
& \quad d(x, y) \geqslant 1 / n\}: 1 \leqslant i \leqslant n\}\} .
\end{aligned}
$$

Then $h=\lim _{n \rightarrow \infty} h_{n}$ is an autohomeomorphism of $X$.

4.6. LemMA. Let A be a (nondiscrete) zero-dimensional, strongly homogeneous space, and suppose the Cantor set is homogeneous with respect to dense copies of $A$. Let $X$ and $Y$ be dense subsets of $C$ such that $X=\bigcup_{i=0}^{\infty} X_{i}, Y=\bigcup_{i=0}^{\infty} Y_{i}, X_{i}$ is nowhere dense closed in $X$, and $Y_{i}$ is nowhere dense closed in $Y$. If either

(1) $X_{i} \approx A \approx Y_{i}$ for each $i \in \omega$, or

(2) $\bar{X}_{i} \backslash X_{i} \approx A \approx \bar{Y}_{i} \backslash Y_{i}$ and $\bar{X}_{i} \backslash X_{i}$ is dense in $\bar{X}_{i}, \bar{Y}_{i} \backslash Y_{i}$ is dense in $\bar{Y}_{i}$ for each $i \in \omega$,

then there exists a homeomorphism $h: C \rightarrow C$ such that $h[X]=Y$.

Proof. Let $U$ be a clopen subset of $C$ such that $U \cap X_{i} \neq \varnothing$. Then in case (1), $U \cap X_{i} \approx A$, and in case (2), $\left(\overline{U \cap X_{i}}\right) \backslash\left(U \cap X_{i}\right)=U \cap\left(\bar{X}_{i} \backslash X_{i}\right) \approx A$, since $A$ is strongly homogeneous. In case (2) also, $\left(\left(\overline{U \cap X_{i}}\right) \backslash\left(U \cap X_{i}\right)\right)^{-}=\left(U \cap\left(\bar{X}_{i} \backslash X_{i}\right)\right)^{-}=$ $\bar{U} \cap X_{i}$, since $\bar{X}_{i} \backslash X_{i}$ is dense in $\bar{X}_{i}$. Similarly, if $V$ is a clopen subset of $C$ such that $V \cap Y_{j} \neq \varnothing$, then in case (1), $V \cap Y_{j} \approx A$, and in case (2), $\left(\overline{V \cap Y_{j}}\right) \backslash\left(V \cap Y_{j}\right) \approx A$ is dense in $\overline{V \cap Y_{j}}$. That there exists a homeomorphism $f: \overline{U \cap X_{i}} \rightarrow \overline{V \cap Y_{j}}$ such that $f\left[U \cap X_{i}\right]=V \cap Y_{j}$ follows, in both cases, from the fact that $C$ is homogeneous with respect to dense copies of $A$.

Now let $M$ be as in the proof of Theorem 4.3. We construct for each $s \in M$ collections $\mathscr{U}(s)=\{U(s, n): n \in \mathbf{N}\}, \mathscr{V}(s)=\{V(s, n): n \in \mathbf{N}\}$ of clopen subsets of $C$, closed nowhere dense subsets $D(s), E(s)$ of $C$, and for each $n \in \mathbf{N}$ a homeomorphism $h_{n}: C \rightarrow C$ such that, if $n=|s|$, then

(1) $X_{n} \subset \bigcup_{|t| \leqslant n} D(t), Y_{n} \subset \bigcup_{|t| \leqslant n} E(t)$;

(2) $D(s) \subset U(s), E(s) \subset V(s), U(\varnothing)=V(\varnothing)=C$;

(3) $h_{n+1}[U(s, i)]=V(s, i)$ for each $i \in \mathbf{N}$;

(4) $h_{n+1}[D(s)]=E(s), h_{n+1}[D(s) \cap X]=E(s) \cap Y$;

(5) $h_{n+1}\left|\bigcup_{|t|<n} D(t)=h_{n}\right| \bigcup_{|t|<n} D(t)$; 
(6) $\{(U(s, i), V(s, i)): i \in \mathbf{N}\}$ is a KR-cover for $(U(s) \backslash D(s), V(s) \backslash E(s)$, $\left.h_{n} \mid D(s)\right)$

(7)

$$
\begin{aligned}
\operatorname{diam}(V)<\varepsilon_{n+1}=\min \left\{2^{-(n+1)}, 3^{-(n+1)} \cdot \min \left\{\operatorname { m i n } \left\{d\left(h_{i}(x), h_{i}(y)\right):\right.\right.\right. & \\
d(x, y) \geqslant 1 /(n+1)\}: 1 \leqslant i \leqslant n+1\}\} & \quad \text { if } V \in \mathscr{V}(s) .
\end{aligned}
$$

Suppose this has been done; we claim that $d\left(h_{n}, h_{n+1}\right)<\varepsilon_{n}$ and that $\lim _{n \rightarrow \infty} h_{n}=h$ : $C \approx C$ has the property that $h[X]=Y$. If $x \in \bigcup_{|t|<n} D(t)$, then by (5), $h_{n+1}(x)=$ $h_{n}(x)$, so $d\left(h_{n}(x), h_{n+1}(x)\right)<\varepsilon_{n}$. If $x \notin \bigcup_{|t|<n} D(t)$, then $x \notin D(\varnothing)$, hence, $x \in$ $U(m)$ for some $m \in \mathbf{N}$, and proceeding inductively, using (6), $x \in U(s, i)$ for some $s \in M$ with $|s|=n-1$, and some $i \in \mathbf{N}$, so by (3), $h_{n}(x) \in V(s, i)$; if $x \in D(s, i)$, then by (4), $h_{n+1}(x) \in E(s, i) \subset V(s, i)$ by (2), and if $x \notin D(s, i)$, then $x \in$ $U(s, i, j)$ for some $j \in \mathbf{N}$, hence by (3), $h_{n+1}(x) \in V(s, i, j) \subset V(s, i)$ by (6). Thus $\left\{h_{n}(x), h_{n+1}(x)\right\} \subset V(s, i) \in \mathscr{V}(s),|s|=n-1$; hence, by $(7), d\left(h_{n}(x), h_{n+1}(x)\right)$ $<\varepsilon_{n}$. Hence, by Theorem 4.5, $\lim _{n \rightarrow \infty} h_{n}=h$ is an autohomeomorphism of $C$. Suppose $x \in X_{n}$; by (1), $x \in D(s)$ for some $s \in M$ with $|s| \leqslant n$; hence, by (4), $h_{|s|+1}(x) \in Y$; by (5), $h_{m}(x)=h_{|s|+1}(x)$ for each $m>|s|+1$, so $h(x)=h_{|s|+1}(x)$, i.e., $h(x) \in Y$. Conversely, if $y \in Y_{n}$, then $y \in E(s)$ for some $s \in M$ with $|s| \leqslant n$; hence, $y=h_{|s|+1}(x)$ for some $x \in D(s) \cap X$; as above, $h(x)=h_{|s|+1}(x)$, so $y \in$ $h[X]$.

We now show how to carry out the induction. First, put $U(\varnothing)=V(\varnothing)=C$, $D(\varnothing)=\bar{X}_{0}, E(\varnothing)=\bar{Y}_{0}$, and let $h^{0}: D(\varnothing) \rightarrow E(\varnothing)$ be a homeomorphism such that $h^{0}\left[X_{0}\right]=Y_{0}$. Let $\mathscr{R}=\left\{R_{i}: i \in \mathbf{N}\right\}, \mathscr{S}=\left\{S_{i}: i \in \mathbf{N}\right\}$ be such that $\left\{\left(R_{i}, S_{i}\right): i \in \mathbf{N}\right\}$ is a $\mathrm{KR}$-cover for $\left(C \backslash D(\varnothing), C \backslash E(\varnothing), h^{0}\right)$; this is possible by Lemma 4.2. Since $R_{i} \approx C \approx S_{i}$, there exists a homeomorphism $h^{i}: R_{i} \rightarrow S_{i}$. Put

$$
h_{1}=\bigcup_{i \in \omega} h^{i}: C \approx C \text {. }
$$

For each $i \in \mathbf{N}$ let $\left\{S(i, 1), \ldots, S\left(i, n_{i}\right)\right\}$ be a disjoint clopen cover of $S_{i}$ by sets of diameter less than $\varepsilon_{1}$ (as in (7)), and for $i \in \mathbf{N}, 1 \leqslant k \leqslant n_{i}$, put

$$
R(i, k)=h_{1}^{-1}[S(i, k)] \text {; }
$$

then $\mathscr{U}(\varnothing)=\left\{R(i, k): i \in \mathbf{N}, 1 \leqslant k \leqslant n_{i}\right\}, \mathscr{V}(\varnothing)=\left\{S(i, k): i \in \mathbf{N}, 1 \leqslant k \leqslant n_{i}\right\}$ can be reindexed as $\{U(n): n \in \mathbf{N}\},\{V(n): n \in \mathbf{N}\}$ such that $\{(U(n), V(n))$ : $n \in \mathbf{N}\}$ is a KR-cover for $\left(C \backslash D(\varnothing), C \backslash E(\varnothing), h_{1} \mid D(\varnothing)\right)$ satisfying $h_{1}[U(n)]=$ $V(n)$. Now suppose $D(s), E(s), \mathscr{U}(s), \mathscr{V}(s)$, and $h_{m}$ have been constructed for $|s|<n(\geqslant 1)$ and $m \leqslant n$. Fix $s \in M$ with $|s|=n-1$. Let

$$
k_{i}=\min \left\{j: U(s, i) \cap X_{j} \neq \varnothing\right\}, \quad l_{i}=\min \left\{j: V(s, i) \cap Y_{j} \neq \varnothing\right\},
$$

and put

$$
D(s, i)=\overline{U(s, i) \cap X_{k_{i}}}, \quad E(s, i)=\overline{V(s, i) \cap Y_{l_{i}}} .
$$

Let $h^{0}(s, i): D(s, i) \rightarrow E(s, i)$ be a homeomorphism such that $h^{0}(s, i)[D(s, i) \cap X]$ $=E(s, i) \cap Y$. Choose $\mathscr{R}(s, i)=\{R(s, i, j): j \in \mathbf{N}\}, \mathscr{S}(s, i)=\{S(s, i, j): j \in \mathbf{N}\}$ such that

$$
\{(R(s, i, j), S(s, i, j)): j \in \mathbf{N}\}
$$


is a KR-cover for $\left(U(s, i) \backslash D(s, i), V(s, i) \backslash E(s, i), h^{0}(s, i)\right)$, and let $h^{j}(s, i)$ : $R(s, i, j) \rightarrow S(s, i, j)$ be a homeomorphism. Put

$$
h(s, i)=\bigcup_{j \in \omega} h^{j}(s, i): U(s, i) \approx V(s, i)
$$

and

$$
h_{n+1}=\bigcup\{h(s, i):|s|=n-1, i \in \mathbf{N}\} \cup h_{n} \mid \bigcup_{|s|<n} D(s) .
$$

As above, we can refine each $\mathscr{S}(s, i)$ by disjoint clopen sets of diameter less than $\varepsilon_{n+1}$ and obtain $\{U(s, i, j): j \in \mathbf{N}\},\{V(s, i, j): j \in \mathbf{N}\}$ such that $\{(U(s, i, j)$, $V(s, i, j)): j \in \mathbf{N}\}$ is a KR-cover for $\left(U(s, i) \backslash D(s, i), V(s, i) \backslash E(s, i), h_{n+1} \mid D(s, i)\right)$ satisfying $h_{n+1}[U(s, i, j)]=V(s, i, j)$. As in the proof of Theorem 4.3, it is easily seen that $h_{n+1}$ is a homeomorphism and the inductive hypotheses are satisfied.

4.7. THEOREM. Let $A$ be a (nondiscrete) zero-dimensional, strongly homogeneous space. If $C$ is homogeneous with respect to dense copies of $A$, then $C$ is also homogeneous with respect to dense copies of $\mathbf{Q} \times A$.

Proof. This follows immediately from Lemma 4.6(1).

5. Hurewicz-type theorems. In this paper we will often be concerned with the construction of closed copies of previously characterized spaces in the space which is yet to be characterized. The first theorem of the type we are looking for was proved by Hurewicz in [13]: he showed that, if a Borel subset $A$ of a compact space $X$ is not complete, then $X$ contains a closed subset $K$ such that $K \cap A \approx \mathbf{Q}$ and $K \backslash A \approx \mathbf{P}$; clearly, the condition that $X$ is not complete is also necessary, since $\mathbf{Q}$ is not complete.

In [21] Saint-Raymond proved a similar theorem: he showed that a Borel subset $A$ of a compact space $X$ is not the union of a $\sigma$-compact subspace and a complete subspace if and only if $A$ contains a closed copy of $\mathbf{Q} \times P$; by Proposition 2.3 this is also equivalent to $X \backslash A$ not being strongly $\sigma$-complete. In [6] the above results were improved, and more Hurewicz-type theorems were proved:

5.1. TheOREM. Let $X$ be a compact space, and let $A$ be a Borel subset of $X$.

(a) $A$ is not complete if and only if $X$ contains a Cantor set $K$ such that $K \cap A \approx \mathbf{Q}$ and $K \backslash A \approx \mathbf{P}$;

(b) $A$ is not complete $\cup$ countable if and only if $X$ contains a Cantor set $K$ such that $K \cap A \approx \mathbf{Q} \times C$ and $K \backslash A \approx \mathbf{P}$;

(c) $A$ is not strongly $\sigma$-complete if and only if $X$ contains a Cantor set $K$ such that $K \cap A \approx T$ and $K \backslash A \approx \mathbf{Q} \times \mathbf{P}$;

(d) $A$ is not strongly $\sigma$-complete $\cup$ countable if and only if $X$ contains a Cantor set $K$ such that $K \cap A \approx S$ and $K \backslash A \approx \mathbf{Q} \times \mathbf{P}$.

The new Hurewicz-type theorems we need in this paper will all be deduced from the following two lemmas; although they are valid for Borel sets in arbitrary compact spaces (see [5]), we will restrict ourselves to Borel subsets of the Cantor set $C$. The proofs are inspired by [21] and [6]. 
5.2. LEMMA. Let $\mathscr{P}$ be a topological property which is closed-hereditary and strongly $\sigma$-additive such that all singletons are $\mathscr{P}$. Let $Z \neq \varnothing$ be a strongly homogeneous space and suppose that if $B$ is a Borel subset of $C$ which is not $\mathscr{P}$, then $C$ contains a Cantor set $K$ such that $K \cap B \approx Z$ and $K \backslash B$ is contained in a $\sigma$-compact subset $G$ of $C$ with the property that $G \cap B$ is $\mathscr{P}$. Then if $A$ is a Borel subset of $C$ which is not $\mathscr{P} \cup$ complete, then $C$ contains a Cantor set $K$ such that $K \cap A \approx \mathbf{Q} \times Z$.

Proof. Since $A$ is a Borel subset of $C$, there exists a continuous surjection $\phi$ : $\mathbf{P} \rightarrow C \backslash A$. Put

$W=\left\{x \in \mathbf{P}:\right.$ there exists a neighbourhood $V_{x}$ of $x$ in $\mathbf{P}$ and a $\sigma$-compact subset $E_{x}$ of $C$ such that $\phi\left[V_{x}\right] \subset E_{x}$ and $E_{x} \cap A$ is $\left.\mathscr{P}\right\}$.

Then $W$ is open in $\mathbf{P}$, so there exist countably many open $V_{i}$ in $\mathbf{P}$ and $\sigma$-compact $E_{i}$ in $C$ such that $W=\cup_{i=1}^{\infty} V_{i}, \phi\left[V_{i}\right] \subset E_{i}$, and $E_{i} \cap A$ is $\mathscr{P}$. Since $E_{i}$ is $\sigma$-compact, we can write

$$
E_{i}=\bigcup_{j=1}^{\infty} K_{j}^{i}
$$

where each $K_{j}^{i}$ is compact; then

$$
E=\bigcup_{i, j=1}^{\infty} K_{j}^{i}
$$

is $\sigma$-compact, and since $K_{j}^{i} \cap A$, being a closed subspace of $E_{i} \cap A$, is $\mathscr{P}$,

$$
E \cap A=\bigcup_{i, j=1}^{\infty}\left(K_{j}^{i} \cap A\right)
$$

is strongly $\sigma-\mathscr{P}$, hence $\mathscr{P}$.

Now suppose $C \backslash A \subset E$; then $A=(E \cap A) \cup(C \backslash E)$ is $\mathscr{P} \cup$ complete, a contradiction. Hence, $F=\mathbf{P} \backslash \phi^{-1}[E]$ is nonempty and complete.

Claim 1. If $\varnothing \neq U$ is open in $F, G \subset C$ is $\sigma$-compact, and $\phi[U] \subset G$, then $G \cap A$ is not $\mathscr{P}$.

Indeed, suppose $G=\bigcup_{i=1}^{\infty} G_{i}$ is $\mathscr{P}$ and $G_{i}$ is compact; then $G_{i} \cap A$ is $\mathscr{P}$, and hence

$$
(G \cup E) \cap A=\bigcup_{i=1}^{\infty}\left(G_{i} \cap A\right) \cup \bigcup_{i, j=1}^{\infty}\left(K_{j}^{i} \cap A\right)
$$

is strongly $\sigma-\mathscr{P}$, hence $\mathscr{P}$. Now let $U^{\prime}$ be open in $\mathbf{P}$ such that $U^{\prime} \cap F=U$; then $\phi\left[U^{\prime}\right]=\phi[U] \cup \phi\left[U^{\prime} \backslash U\right] \subset G \cup E$, and since $U^{\prime} \not \subset W,(G \cup E) \cap A$ is not $\mathscr{P}$, a contradiction.

From this claim it follows that if $\varnothing \neq U$ is open in $F$, then $\overline{\phi[U]} \cap A$ is not $\mathscr{P}$. By the Cantor-Bendixson theorem, we can write $\overline{\phi[U]}=L \cup M$, where $L$ is compact dense in itself, or empty, and $M$ is countable and disjoint from $L$. Suppose $L=\varnothing ;$ then $\overline{\phi[U]} \cap A$ is countable and, hence, $\mathscr{P}$, since singletons are $\mathscr{P}$ and $\mathscr{P}$ is strongly $\sigma$-additive, a contradiction. So $L \approx C$. By a similar argument, $L \cap A$ is not $\mathscr{P}$, and thus $L$, and hence $\overline{\phi[U]}$ contains a Cantor set $K$ such that $K \cap A \approx Z$ and $K \backslash A$ is contained in a $\sigma$-compact subset $G$ of $L$ (and hence of $C$ ) with the property that $G \cap A$ is $\mathscr{P}$. 
Now, as in the proof of Theorem 4.3, let $M$ be the set of all finite sequences of natural numbers, and for $s=\left(s_{1}, \ldots, s_{k}\right)$, put $|s|=k, \nu(s)=s_{1}+\cdots+s_{k}$, and $|\varnothing|=\nu(\varnothing)=0$. We will construct Cantor sets $K_{s}$ in $C$, open subsets $W_{s}$ of $F$, and open subsets $U_{s}$ of $C$, for each $s \in M$, such that the following hold:

(1) $K_{s} \subset \overline{\phi\left[W_{s}\right]} \subset U_{s}$

(2) for each $n \in \mathbf{N}, \bar{U}_{s, n} \cap K_{s}=\varnothing$;

(3) for each $n, m \in \mathbf{N}, \bar{U}_{s, n} \cap \bar{U}_{s, m}=\varnothing$ if $n \neq m$;

(4) for each $n \in \mathbf{N}, \bar{W}_{s, n} \subset W_{s}$;

(5) for each $n \in \mathrm{N}, \bar{U}_{s, n} \subset U_{s}$;

(6) $\operatorname{diam}\left(W_{s}\right) \leqslant 2^{-|s|}$ (with respect to a complete metric on $F$ );

(7) $\operatorname{diam}\left(U_{s}\right) \leqslant 2^{-\nu(s)}$;

(8) for each $n \in \mathbf{N}, d\left(K_{s}, K_{s, n}\right) \leqslant 2^{1-\nu(s, n)}$;

(9) $K_{s} \cap A \approx Z$, and $K_{s} \backslash A \subset G_{s}$ for some $\sigma$-compact subset $G_{s}$ of $C$ such that $G_{s} \cap A$ is $\mathscr{P}$.

We use induction on $|s|$. First, put $W_{\varnothing}=F, U_{\varnothing}=C$. Then $\overline{\phi\left[W_{\varnothing}\right]}$ contains a Cantor set $K_{\varnothing}$ such that $K_{\varnothing} \cap A \approx Z$ and $K_{\varnothing} \backslash A \subset G_{\varnothing}$ for some $\sigma$-compact subset $G_{\varnothing}$ of $C$ such that $G_{\varnothing} \cap A$ is $\mathscr{P}$. Then (1) and (9) are satisfied, and so are (6) and (7) since all metrics are assumed to be bounded by 1 .

Next, suppose that $K_{s}, W_{s}$, and $U_{s}$ have been constructed for $|s| \leqslant k$, and fix $s \in M$ with $|s|=k$.

Claim 2. $K_{s}$ is nowhere dense in $K_{s} \cup \phi\left[W_{s}\right]$.

We distinguish two cases.

Case 1. Let $y \in K_{s} \cap \phi\left[W_{s}\right]$, say $y=\phi(x)$, with $x \in W_{s}$, and let $U$ be an open neighbourhood of $y$ in $K_{s} \cup \phi\left[W_{s}\right]$. By continuity of $\phi: W_{s} \rightarrow \phi\left[W_{s}\right]$ there exists an open neighbourhood $V$ of $x$ in $W_{s}$ such that $\phi[V] \subset U \cap \phi\left[W_{s}\right]$. Suppose $U \cap \phi\left[W_{s}\right]$ $\subset K_{s}$. Then $\phi[V] \subset K_{s} \backslash A \subset G_{s}$, and $G_{s}$ is a $\sigma$-compact subset of $C$ such that $G_{s} \cap A$ is $\mathscr{P}$ (apply (9)). Since $V$ is a nonempty open subset of $F$, this contradicts Claim 1, and hence $U \cap\left(\phi\left[W_{s}\right] \backslash K_{s}\right) \neq \varnothing$.

Case 2. Let $y \in K_{s} \backslash \phi\left[W_{s}\right]$, and let $U$ be an open neighbourhood of $y$ in $K_{s} \cup \phi\left[W_{s}\right]$. Since $K_{s} \subset \overline{\phi\left[W_{s}\right]}$ by (1), $U \cap \phi\left[W_{s}\right] \neq \varnothing$, say $z \in U \cap \phi\left[W_{s}\right]$. If $z \notin K_{s}$, we are done, and if $z \in K_{s}$, then $z \in K_{s} \cap \phi\left[W_{s}\right]$, and Case 1 applies.

This proves the claim.

By this claim we can apply Lemma 2.5 to obtain a countable discrete subset $D_{s}=\left\{y_{s, n}: n \in \mathbf{N}\right\}$ of $\phi\left[W_{s}\right] \backslash K_{s}$ such that $\bar{D}_{s}=D_{s} \cup K_{s}$, and $d\left(y_{s, n}, K_{s}\right) \leqslant 2^{-\nu(s, n)}$ for each $n \in \mathbf{N}$. Now let $U_{s, n}$ be an open neighbourhood of $y_{s, n}$ such that $\bar{U}_{s, n} \subset U_{s}$, $\bar{U}_{s, n} \cap K_{s}=\varnothing, \bar{U}_{s, n} \cap \bar{U}_{s, m}^{s, n}=\varnothing$ if $n \neq m$, and $\operatorname{diam}\left(U_{s, n}\right) \leqslant 2^{-\nu(s, n)}$ for each $n$, $m \in \mathbf{N}$. Since $y_{s, n} \in \phi\left[W_{s}\right], y_{s, n}=\phi\left(x_{s, n}\right)$ for some $x_{s, n} \in W_{s}$; hence, there is an open neighbourhood $W_{s, n}$ of $x_{s, n}$ in $F$ such that $\bar{W}_{s, n} \subset W_{s}, \operatorname{diam}\left(W_{s, n}\right) \leqslant 2^{-|s|-1}$, and $\overline{\phi\left[W_{s, n}\right]} \subset U_{s, n}$. Then $\overline{\phi\left[W_{s, n}\right]}$ contains a Cantor set $K_{s, n}$ such that $K_{s, n} \cap A \approx$ $Z$, and $K_{s, n} \backslash A \subset G_{s, n}$ for some $\sigma$-compact subset $G_{s, n}$ of $C$ such that $G_{s, n} \cap A$ is $\mathscr{P}$. Then (1)-(7) and (9) are satisfied. To prove (8) note that

$$
d\left(K_{s}, K_{s, n}\right) \leqslant d\left(K_{s}, U_{s, n}\right)+\operatorname{diam}\left(U_{s, n}\right) \leqslant d\left(K_{s}, y_{s, n}\right)+2^{-\nu(s, n)} \leqslant 2^{1-\nu(s, n)} .
$$

This completes the induction. For each $k \in \omega$ put

$$
B_{k}=\bigcup\left\{K_{s}:|s| \leqslant k\right\} \text {. }
$$


By induction we will show that $B_{k} \approx C$ for each $k \in \omega$. For $k=0$ this is clear, so suppose $B_{j} \approx C$ for $j \leqslant k(\geqslant 1)$. By Theorem 2.4 it suffices to show that $B_{k+1}$ is closed in $C$. For each $\varepsilon>0$ put

$$
B_{k}^{\varepsilon}=\left\{x \in C: d\left(x, B_{k}\right) \leqslant \varepsilon\right\},
$$

and

$$
M_{k}^{\varepsilon}=\left\{s \in M:|s|=k+1, K_{s} \not \subset B_{k}^{\varepsilon}\right\}
$$

Then each $B_{k}^{\varepsilon}$ is compact, and $M_{k}^{\varepsilon}$ is finite by (1), (7), and (8). Since $B_{k}$ is compact by the inductive hypothesis, we have

$$
B_{k}=\bigcap_{\varepsilon>0} B_{k}^{\varepsilon}
$$

whence $B_{k+1}=\bigcap_{\varepsilon>0}\left(B_{k}^{\varepsilon} \cup \cup\left\{K_{s}: s \in M_{k}^{\varepsilon}\right\}\right)$ is compact, being the intersection of compacta. So $B_{k+1} \approx C$. Now put

$$
K=\left(\bigcup_{i=0}^{\infty} B_{i}\right)^{-}
$$

then clearly $K \approx C$. We claim that $K \cap A=\bigcup_{i=0}^{\infty}\left(B_{i} \cap A\right)$. Suppose to the contrary that $x \in(K \cap A) \backslash \bigcup_{i=0}^{\infty} B_{i}$, and fix $i \in \omega$. Since $x \notin B_{i}, x \notin B_{i}^{\varepsilon}$ for some $\varepsilon>0$. From (1) and (4) it follows that

$$
\bigcup_{j=0}^{\infty} B_{j} \subset B_{i} \cup \bigcup_{|s|=i} \overline{\phi\left[W_{s}\right]},
$$

and from (1) and (7) that $\overline{\phi\left[W_{s}\right]} \subset B_{i}^{\varepsilon}$ for all but finitely many $s \in M$ with $|s|=i$. Hence, for some finite $M_{0} \subset\{s \in M:|s|=i\}$, we have

$$
K \subset B_{i}^{\varepsilon} \cup \bigcup_{s \in M_{0}} \overline{\phi\left[W_{s}\right]} .
$$

Then $x \in \overline{\phi\left[W_{s}\right]}$ for some $s \in M_{0}$, and this $s$ is unique with $|s|=i$ by (3) and (5). So, by (4), there exists an infinite sequence $\sigma$ of natural numbers such that

$$
x \in \bigcap\left\{\overline{\phi\left[W_{s}\right]}: s \text { is an initial segment of } \sigma\right\},
$$

which is a one-point set by (1) and (7). Also,

$$
\bigcap\left\{\bar{W}_{s}: s \text { is an initial segment of } \sigma\right\}=\bigcap\left\{W_{s}: s \text { is an initial segment of } \sigma\right\}
$$

is a one-point set by (5) and by completeness of the metric on $F$. Hence, if

$$
z \in \cap\left\{W_{s}: s \text { is an initial segment of } \sigma\right\},
$$

then $\phi(z)=x$, so $x \in \phi[\mathbf{P}]=C \backslash A$, a contradiction since $x \in K \cap A$. So

$$
K \cap A=\bigcup_{i=0}^{\infty}\left(B_{i} \cap A\right)=\bigcup_{s \in M}\left(K_{s} \cap A\right) .
$$

Obviously, $K_{s} \cap A$ is closed in $K \cap A$, so by Theorem 4.3 it suffices to show that $K_{s} \cap A$ is nowhere dense in $K \cap A$ in order to prove that $K \cap A \approx \mathbf{Q} \times Z$. So let $x \in K_{s} \cap A$ and $\varepsilon>0$. Choose $n \in \mathbf{N}$ so large that $2^{-\nu(s, n)}<\varepsilon / 2$. Since $\bar{D}_{s}=D_{s} \cup$ $K_{s}, y_{s, m} \in B\left(x, 2^{-\nu(s, n)}\right)$ for some $m>n$; and since $y_{s, m} \in U_{s, m}$ and $\operatorname{diam}\left(U_{s, m}\right) \leqslant$ $2^{-\nu(s, m)}$, we have $U_{s, m} \subset B(x, \varepsilon)$, so $K_{s, m} \subset B(x, \varepsilon)$. By (2), $K_{s, m} \cap K_{s}=\varnothing$, so

$$
B(x, \varepsilon) \cap\left((K \cap A) \backslash\left(K_{s} \cap A\right)\right) \supset\left(K_{s, m} \cap A\right) \neq \varnothing .
$$


5.3. LeMmA. Let $\mathscr{P}$ be as in Lemma 5.2, and suppose that for each $i \in \omega, Y_{i}$ and $Z_{i}$ are noncompact strongly homogeneous spaces. Furthermore, assume that if $B$ is a Borel subset of $C$ which is not $\mathscr{P}$, then $C$ contains, for each $i \in \omega, a$ Cantor set $K_{i}$ such that

(I) $K_{i} \backslash B \approx Z_{i}$;

(II) $K_{i} \cap B$ is $\mathscr{P}$;

(III) $K_{i} \cap B \approx Y_{i}$.

Then if $A$ is a Borel subset of $C$ which is not $\mathscr{P}, C$ contains Cantor sets $B_{i}$, for $i \in \omega$, such that $K=\left(\cup_{i=0}^{\infty} B_{i}\right)^{-} \approx C$ and, for each $i \in \omega$,

(i) $B_{i}$ is nowhere dense in $K$;

(ii) $K \backslash A=\bigcup_{i=0}^{\infty}\left(B_{i} \backslash A\right)$;

(iii) $B_{0} \backslash A \approx Z_{0}$ and $\left(B_{i+1} \backslash B_{i}\right) \backslash A \approx Z_{i+1}$;

(iv) $B_{0} \cap A \approx Y_{0}$ and $\left(B_{i+1} \backslash B_{i}\right) \cap A \approx Y_{i+1}$.

If the Cantor sets $K_{i}$ only satisfy (I) and (II), we can construct $K$ satisfying (i)-(iii).

Proof. Since $A$ is a Borel subset of $C$, there exists a continuous surjection $\phi$ : $\mathbf{P} \rightarrow A$. Put

$$
\begin{aligned}
& W=\left\{x \in \mathbf{P}: \exists \text { a neighbourhood } V_{x} \text { of } x \text { in } \mathbf{P}\right. \text {, and a } \\
& \left.\quad \sigma \text {-compact subset } E_{x} \text { of } C \text {, s.t. } \phi\left[V_{x}\right] \subset E_{x} \text {, and } E_{x} \cap A \text { is } \mathbf{P}\right\} .
\end{aligned}
$$

Then $W$ is open in $\mathbf{P}$, so there exist countably many open $V_{i}$ in $\mathbf{P}$ and $\sigma$-compact $E_{i}$ in $C$ such that $W=\bigcup_{i=1}^{\infty} V_{i}, \phi\left[V_{i}\right] \subset E_{i}$, and $E_{i} \cap A$ is $\mathscr{P}$. Put $E=\cup_{i=1}^{\infty} E_{i}$; then it follows as in Lemma 5.2 that $E \cap A$ is $\mathscr{P}$. Now suppose $A \subset E$; then $A=E \cap A$ is $\mathscr{P}$, a contradiction. Hence, $F=\mathbf{P} \backslash \phi^{-1}[E]$ is nonempty and complete.

As in Lemma 5.2, we can show that if $U \neq \varnothing$ is open in $F$, then $\overline{\phi[U]}$ is not $\mathscr{P}$; we can then show that $\overline{\phi[U]}$ contains Cantor sets $K_{i}$ satisfying (I)-(III). Construct $K_{s}, W_{s}$ and $U_{s}$ as above, satisfying (1)-(8),

(9)' $K_{s} \backslash A \approx Z_{|s|}$ and $K_{s} \cap A$ is $\mathscr{P}$, and, if (III) holds,

(10) $K_{s} \cap A \approx Y_{|s|}$.

Since this time $\phi$ is a mapping onto $A$, we find that $K \backslash \bigcup_{i=0}^{\infty} B_{i} \subset \phi[\mathrm{P}]=A$, so that $K \backslash A=\bigcup_{i=0}^{\infty}\left(B_{i} \backslash A\right)$. Since $B_{i+1} \backslash B_{i}=\bigcup\left\{K_{s}:|s|=i+1\right\}$, and since $K_{s} \subset U_{s}$, and $U_{s} \cap U_{t}=\varnothing$ for all $s, t \in M$ with $|s|=|t|$, we have

$$
\left(B_{i+1} \backslash B_{i}\right) \backslash A=\bigcup\left\{K_{s} \backslash A:|s|=i+1\right\} \approx \mathbf{N} \times Z_{i+1} \approx Z_{i+1},
$$

since $Z_{i+1}$ is noncompact and strongly homogeneous. If (III) holds, then ( $\left.B_{i+1} \backslash B_{i}\right)$ $\cap A \approx Y_{i+1}$ follows in the same way.

6. Finite unions of strongly $\sigma$-complete spaces. We recall the topological properties $\mathscr{P}_{n}^{(i)}$ from Definition 3.5:

$X$ is $\mathscr{P}_{4 k}$ iff $X$ is $\Sigma_{k} \cup$ complete;

$X$ is $\mathscr{P}_{4 k+1}$ iff $X$ is $\Sigma_{k+1}$;

$X$ is $\mathscr{P}_{4 k+2}^{1+1}$ iff $X$ is $\Sigma_{k} \cup$ complete $\cup$ countable;

$X$ is $\mathscr{P}_{4 k+3}^{1}$ iff $X$ is $\Sigma_{k+1} \cup$ countable;

$X$ is $\mathscr{P}_{4 k+2}^{2+2}$ iff $X$ is $\Sigma_{k} \cup$ complete $\cup \sigma$-compact;

$X$ is $\mathscr{P}_{4 k+3}^{2}$ iff $X$ is $\Sigma_{k+1} \cup \sigma$-compact. 
Note the following equivalences:

$$
\begin{aligned}
& \mathscr{P}_{4(k+1)} \equiv \mathscr{P}_{4 k+1} \cup \text { complete; } \\
& \mathscr{P}_{4 k+2}^{1} \equiv \mathscr{P}_{4 k}^{1} \cup \text { countable } \equiv \mathscr{P}_{4(k-1)+3}^{1} \cup \text { complete; } \\
& \mathscr{P}_{4 k+3}^{1} \equiv \mathscr{P}_{4 k+1} \cup \text { countable; } \\
& \mathscr{P}_{4 k+2}^{2} \equiv \mathscr{P}_{4 k} \cup \sigma \text {-compact } \equiv \mathscr{P}_{4(k-1)+3}^{2} \cup \text { complete; } \\
& \mathscr{P}_{4 k+3}^{2} \equiv \mathscr{P}_{4 k+1} \cup \text { o-compact. }
\end{aligned}
$$

6.1. Lemma. (a) For each $n \in \omega$ and each $i \in\{1,2\}, \mathscr{P}_{n}^{(i)}$ is closed-hereditary.

(b) For each $n \in \omega, \mathscr{P}_{n}^{(1)}$ is hereditary with respect to $G_{\delta^{-}}$-subsets.

Proof. (a) It suffices to show that a closed subset of a strongly $\sigma$-complete space is strongly $\sigma$-complete. But if $X=\bigcup_{i=1}^{\infty} X_{i}$, with $X_{i}$ closed in $X$ and complete, then for a closed $A \subset X, A=\bigcup_{i=1}^{\infty}\left(X_{i} \cap A\right), X_{i} \cap A$ is closed in $A$ and also in $X_{i}$, so $X_{i} \cap A$ is complete.

The proof of (b) is similar; $\mathscr{P}_{4 k+2}^{2}$ and $\mathscr{P}_{4 k+3}^{2}$ are not $G_{\delta}$-hereditary since $\sigma$ compactness is not.

6.2. LeMMA. Let $X$ be a topological space.

(a) For each $k \in \omega, X$ is $\mathscr{P}_{4 k+1}$ if and only if $X$ is strongly $\sigma-\mathscr{P}_{4 k}$.

(b) For each $k \in \omega$ and each $i \in\{1,2\}, X$ is $\mathscr{P}_{4 k+3}^{i}$ if and only if $X$ is strongly $\sigma-\mathscr{P}_{4 k+2}^{i}$.

Proof. (a) For $k=0$ this is clear, so suppose it is true for $k<n(\geqslant 1)$. If $X$ is $\mathscr{P}_{4 n+1}$, then $X$ is $\mathscr{P}_{4(n-1)+1} \cup \Sigma_{1}$, so by the inductive hypothesis we can write $X=A \cup B$, where $A=\bigcup_{i=1}^{\infty} A_{i}$, with $A_{i}$ closed in $A$ and $\mathscr{P}_{4(n-1)}$, and $B=\bigcup_{i=1}^{\infty} B_{i}$, with $B_{i}$ closed in $B$ and complete. Then for each $i, \overline{A_{i}}=A_{i} \cup\left(\bar{A}_{i} \cap B\right)$ is $\mathscr{P}_{4(n-1)} \cup \Sigma_{1}$ by Lemma 6.1 ; i.e., $\bar{A}_{i}$ is $\Sigma_{n-1} \cup$ complete $\cup \Sigma_{1}$ is $\Sigma_{n} \cup$ complete is $\mathscr{P}_{4 n}$; and $\bar{B}_{i}=B_{i} \cup\left(\bar{B}_{i} \cap A\right)$ is complete $\cup \mathscr{P}_{4(n-1)+1}$ is $\mathscr{P}_{4 n}$. Thus, $X=\cup_{i=1}^{\infty} \bar{A}_{i} \cup \cup_{i=1}^{\infty} \bar{B}_{i}$ is strongly $\sigma-\mathscr{P}_{4 n}$.

Conversely, suppose $X=\cup_{i=1}^{\infty} X_{i}$, where $X_{i}$ is closed in $X$ and $\mathscr{P}_{4 n}$; i.e., $X_{i}$ is $\mathscr{P}_{4(n-1)+1} \cup$ complete; by the inductive hypothesis

$$
X_{i}=\bigcup_{j=1}^{\infty} A_{j}^{i} \cup G_{i},
$$

where $A_{j}^{i}$ is closed in $\bigcup_{j=1}^{\infty} A_{j}^{i}$ and $\mathscr{P}_{4(n-1)}$, and $G_{i}$ is complete. Put

$$
A=\bigcup_{i, j=1}^{\infty} \overline{A_{j}^{i}} \text {. }
$$

Since $\overline{A_{j}^{i}} \subset \bar{X}_{i}=X_{i}$, we have

$$
\overline{A_{j}^{i}}=A_{j}^{i} \cup\left(\overline{A_{j}^{i}} \cap G_{i}\right)
$$

is $\mathscr{P}_{4(n-1)} \cup$ complete is $\mathscr{P}_{4(n-1)}$, so $A$ is strongly $\sigma-\mathscr{P}_{4(n-1)}$, hence, $\mathscr{P}_{4(n-1)+1}$ by the inductive hypothesis. Also, $A$ is an $F_{\sigma}$ in $X$, so each $G_{i} \backslash A$ is complete; if

$$
x \in \overline{G_{i} \backslash A} \cap \bigcup_{j=1}^{\infty}\left(G_{j} \backslash A\right),
$$


then $x \in \bar{G}_{i} \subset X_{i}, x \notin A$, so $x \in G_{i}$; hence $\bigcup_{j=1}^{\infty}\left(G_{j} \backslash A\right)$ is strongly $\sigma$-complete. Thus,

$$
X=A \cup \bigcup_{j=1}^{\infty}\left(G_{j} \backslash A\right)
$$

is $\mathscr{P}_{4(n-1)+1} \cup \Sigma_{1}$ is $\mathscr{P}_{4 n+1}$.

(b) For $i=1$ : By (a), $X$ is $\mathscr{P}_{4 k+3}^{1}$ if and only if $X$ is strongly $\sigma-\mathscr{P}_{4 k} \cup$ countable. So suppose $X=\cup_{i=1}^{\infty} A_{i} \cup B$, where $A_{i}$ is closed in $\bigcup_{i=1}^{\infty} A_{i}$ and $\mathscr{P}_{4 k}$, and $B$ is countable. Then $\overline{A_{i}}=A_{i} \cup\left(\overline{A_{i}} \cap B\right)$ is $\mathscr{P}_{4 k} \cup$ countable is $\mathscr{P}_{4 k+2}^{1}$. Since $\{b\}$ is $\mathscr{P}_{4 k+2}^{1}$ for each $b \in B$,

$$
X=\bigcup_{i=1}^{\infty} \overline{A_{i}} \cup \bigcup_{b \in B}\{b\}
$$

is strongly $\sigma-\mathscr{P}_{4 k+2}^{1}$.

Conversely, suppose $X=\bigcup_{i=1}^{\infty} X_{i}$, with $X_{i}$ closed in $X$ and $\mathscr{P}_{4 k+2}^{1}$, i.e., $X_{i}=A_{i} \cup B_{i}$, where $A_{i}$ is $\mathscr{P}_{4 k}$ and $B_{i}$ is countable. Put $B=\bigcup_{i=1}^{\infty} B_{i}$; then each $A_{i} \backslash B$ is $\mathscr{P}_{4 k}$ by Lemma 6.1. Also, if

$$
x \in \overline{A_{i} \backslash B} \cap \bigcup_{j=1}^{\infty}\left(A_{j} \backslash B\right),
$$

then $x \in \overline{A_{i}} \subset \bar{X}_{i}=X_{i}, x \notin B$, so $x \in A_{i} \backslash B$. Thus, $\bigcup_{j=1}^{\infty}\left(A_{j} \backslash B\right)$ is strongly $\sigma-\mathscr{P}_{4 k}$, and

$$
X=\bigcup_{i=1}^{\infty}\left(A_{i} \backslash B\right) \cup B
$$

is strongly $\sigma-\mathscr{P}_{4 k} \cup$ countable. The case $i=2$ is similar.

6.3. Corollary. For odd $n \in \mathbf{N}$ and each $i \in\{1,2\}, \mathscr{P}_{n}^{(i)}$ is a strongly $\sigma$-additive property.

6.4. LemMA. Let $X$ be compact, and let $A$ be a subset of $X$. Then for each $k \in \omega$,

(a) $A$ is $\mathscr{P}_{4 k}$ if and only if $X \backslash A$ is $\mathscr{P}_{4(k-1)+3}^{2}$,

(b) $A$ is $\mathscr{P}_{4 k+1}$ if and only if $X \backslash A$ is $\mathscr{P}_{4 k+2}^{2}$.

Proof. We prove (a) and (b) simultaneously by induction on $k$. For $k=0$, (a) is trivial, and (b) follows from Proposition 2.3. So suppose (a) and (b) have been proved for $k<n(\geqslant 1)$, and let $A$ be $\mathscr{P}_{4 n}$, i.e., $A$ is $\mathscr{P}_{4(n-1)+1} \cup$ complete. By Lemma 6.2 we can write $A=\bigcup_{i=1}^{\infty} A_{i} \cup B$, where $A_{i}$ is closed in $\bigcup_{i=1}^{\infty} A_{i}$ and $\mathscr{P}_{4(n-1)}$, and $B$ is complete. Since $\mathrm{Cl}_{A}\left(A_{i}\right)=A_{i} \cup\left(\overline{A_{i}} \cap B\right)$ is $\mathscr{P}_{4(n-1)} \cup$ complete is $\mathscr{P}_{4(n-1)}$, we may assume that each $A_{i}$ is closed in $A$. Then

$$
X \backslash A=\bigcup_{i=1}^{\infty}\left(\overline{A_{i}} \backslash A_{i}\right) \cup\left(X \backslash \bigcup_{i=1}^{\infty} \overline{A_{i}}\right) \backslash B .
$$

By the inductive hypothesis, we have $\overline{A_{i}} \backslash A_{i}$ is $\mathscr{P}_{4(n-2)+3}^{2}$; hence, by Corollary 6.3, $\bigcup_{i=1}^{\infty}\left(\overline{A_{i}} \backslash A_{i}\right)$ is also $\mathscr{P}_{4(n-2)+3}^{2}$; and $\left(X \backslash \bigcup_{i=1}^{\infty} \overline{A_{i}}\right) \backslash B$ is complete $\backslash$ complete is strongly $\sigma$-complete. So $X \backslash A$ is $\Sigma_{n-1} \cup \sigma$-compact $\cup \Sigma_{1}$ is $\Sigma_{n} \cup \sigma$-compact is $\mathscr{P}_{4(n-1)+3}^{2}$. 
Conversely, if $X \backslash A$ is $\mathscr{P}_{4(n-1)+3}^{2}$, then by Lemma 6.2, $X \backslash A=\bigcup_{i=1}^{\infty} A_{i}$, where $A_{i}$ is closed in $X \backslash A$ and $\mathscr{P}_{4(n-1)+2}^{2}$. Hence,

$$
A=\bigcup_{i=1}^{\infty}\left(\overline{A_{i}} \backslash A_{i}\right) \cup X \backslash \bigcup_{i=1}^{\infty} \overline{A_{i}} .
$$

By the inductive hypothesis, we have $\overline{A_{i}} \backslash A_{i}$ is $\mathscr{P}_{4(n-1)+1}$; hence, by Corollary 6.3 , $\bigcup_{i=1}^{\infty}\left(\bar{A}_{i} \backslash A_{i}\right)$ is also $\mathscr{P}_{4(n-1)+1}$, and $X \backslash \bigcup_{i=1}^{\infty} \bar{A}_{i}$ is complete. Thus, $A$ is $\mathscr{P}_{4(n-1)+1} \cup$ complete is $\mathscr{P}_{4 n}$.

To prove (b) suppose $A=\bigcup_{i=1}^{\infty} A_{i}$, where $A_{i}$ is closed in $A$ and $\mathscr{P}_{4 n}$; then

$$
X \backslash A=\bigcup_{i=1}^{\infty}\left(\overline{A_{i}} \backslash A_{i}\right) \cup X \backslash \bigcup_{i=1}^{\infty} \overline{A_{i}}
$$

is strongly $\sigma-\mathscr{P}_{4(n-1)+3}^{2} \cup$ complete by the inductive hypothesis; hence, $\mathscr{P}_{4(n-1)+3}^{2} \cup$ complete is $\mathscr{P}_{4 n+2}^{2}$.

Conversely, if $X \backslash A$ is $\mathscr{P}_{4 n+2}^{2}$ is $\mathscr{P}_{4(n-1)+3}^{2} \cup$ complete, then, by Lemma 6.2, we can write $X \backslash A=\bigcup_{i=1}^{\infty} A_{i} \cup B$, where $A_{i}$ is closed in $\bigcup_{i=1}^{\infty} A_{i}$ and $\mathscr{P}_{4(n-1)+2}^{2}$, and $B$ is complete. Since $\mathrm{Cl}_{X \backslash A}\left(A_{i}\right)=A_{i} \cup\left(\bar{A}_{i} \cap B\right)$ is $\mathscr{P}_{4(n-1)+2}^{2} \cup$ complete is $\mathscr{P}_{4(n-1)+2}^{2}$, we may assume that each $A_{i}$ is closed in $X \backslash A$. Then

$$
A=\bigcup_{i=1}^{\infty}\left(\overline{A_{i}} \backslash A_{i}\right) \cup\left(X \backslash \bigcup_{i=1}^{\infty} \overline{A_{i}}\right) \backslash B
$$

is strongly $\sigma-\mathscr{P}_{4(n-1)+1} \cup \Sigma_{1}$ is $\mathscr{P}_{4(n-1)+1} \cup \Sigma_{1}$ is $\Sigma_{n+1}$ is $\mathscr{P}_{4 n+1}$.

6.5. LEMMA. Between the properties $\mathscr{P}_{n}^{(i)}$, the following implications hold:

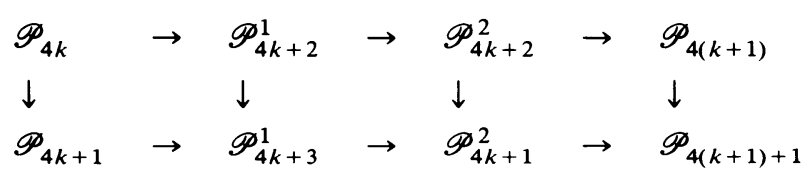

Corresponding to each property $\mathscr{P}_{n}^{(i)}$ we now define a class of spaces $\mathscr{X}_{n}^{(i)}$ and show that, up to homeomorphism, each $\mathscr{X}_{n}^{(i)}$ contains exactly one element.

6.6. Definition. (a) $\mathscr{X}_{-1}^{1}=\{\mathbf{Q}\} ; \mathscr{X}_{-1}^{2}=\{\mathbf{Q} \times C\} ; \mathscr{X}_{0}=\{\mathbf{P}\}$.

(b) Let $X$ be zero-dimensional; then for each $k \in \omega$,

$X \in \mathscr{X}_{4(k+1)}$ iff $X$ is $\mathscr{P}_{4(k+1)}$, nowhere $\mathscr{P}_{4 k+2}^{2}$, nowhere $\mathscr{P}_{4 k+1}$;

$X \in \mathscr{X}_{4 k+1}$ iff $X$ is $\mathscr{P}_{4 k+1}$, nowhere $\mathscr{P}_{4 k}$, nowhere $\mathscr{P}_{4(k-1)+3}^{2}$;

$X \in \mathscr{X}_{4 k+2}^{1}$ iff $X$ is $\mathscr{P}_{4 k+2}^{1}$, nowhere $\mathscr{P}_{4 k}$, nowhere $\mathscr{P}_{4(k-1)+3}^{2}$;

$X \in \mathscr{X}_{4 k+2}^{2}$ iff $X$ is $\mathscr{P}_{4 k+2}^{2}$, nowhere $\mathscr{P}_{4 k+2}^{1}$, nowhere $\mathscr{P}_{4(k-1)+3}^{2}$;

$X \in \mathscr{X}_{4 k+3}^{1}$ iff $X$ is $\mathscr{P}_{4 k+3}^{1}$, nowhere $\mathscr{P}_{4 k+2}^{2}$, nowhere $\mathscr{P}_{4 k+1}$;

$X \in \mathscr{X}_{4 k+3}^{2}$ iff $X$ is $\mathscr{P}_{4 k+3}^{2}$, nowhere $\mathscr{P}_{4 k+2}^{2}$, nowhere $\mathscr{P}_{4 k+3}^{1}$.

Observe that if $X \in \mathscr{X}_{n}^{(i)}$, then $X$ is nowhere locally compact; so $X$ can be embedded in $C$ as a dense and codense subset.

6.7. Lemma. Let $n \in \omega, i \in\{1,2\}$, and $X \in \mathscr{X}_{n}^{(i)}$.

(a) If $n$ is odd, then $X$ is not Baire.

(b) If $n$ is even, then $X$ contains a dense complete subset and, hence, is Baire.

Proof. If $n$ is odd, then $X$ is strongly $\sigma-\mathscr{P}_{n-1}^{(i)}$ and nowhere $\mathscr{P}_{n-1}^{(i)}$, so $X$ is not Baire. If $n=0$, then $X$ itself is complete. If $n=4 k>0$, then $X$ is $\mathscr{P}_{4 k}$, hence $X=A \cup B$, 
where $A$ is $\Sigma_{k}$ and $B$ is complete; since $X$ is nowhere $\mathscr{P}_{4(k-1)+1}, X$ is nowhere $\Sigma_{k}$, hence $B$ is dense in $X$. The other cases are proved similarly.

The following lemma gives alternative descriptions of $\mathscr{X}_{n}^{(i)}$ for even $n$; in some cases these are easier to use than those of Definition 6.6.

6.8. Lemma. Let $X$ be zero-dimensional. Then for each $k \in \omega$ :

(a) $X \in \mathscr{X}_{4 k}$ iff $X$ is $\mathscr{P}_{4 k}$ and nowhere $\mathscr{P}_{4(k-1)+3}^{2}$.

(b) $X \in \mathscr{X}_{4 k+2}^{1}$ iff $X$ is $\mathscr{P}_{4 k+2}^{1}$ and nowhere $\mathscr{P}_{4 k+1}$.

(c) $X \in \mathscr{X}_{4 k+2}^{2}$ iff $X$ is $\mathscr{P}_{4 k+2}^{2}$ and nowhere $\mathscr{P}_{4 k+3}^{1}$.

Proof. We only have to consider the "nowhere" properties. The "if" part follows immediately from Lemma 6.5. Part (a) of the lemma follows from Theorem 2.4 for $k=0$, so suppose $k>0$ and $X \in \mathscr{X}_{4 k}$. Let $U$ be open in $X$ and suppose $U$ is $\mathscr{P}_{4(k-1)+3}^{2}$; then, by Lemma $6.2, U$ is strongly $\sigma-\mathscr{P}_{4(k-1)+2}^{2}$. But $X$ is nowhere $\mathscr{P}_{4(k-1)+2}^{2}$, hence $U$ is not Baire, contradicting Lemma 6.7. For part (b) suppose $X \in \mathscr{X}_{4 k+2}^{1}, U$ open in $X$, and $\mathscr{P}_{4 k+1}$. Again, since $X$ is nowhere $\mathscr{P}_{4 k}, U$ is not Baire, a contradiction. Part (c) is similar.

We will now prove that each of the classes defined above contains at least one element.

6.9. Lemma. Let $n \in \omega$ be even, $i \in\{1,2\}$, and $X \in \mathscr{X}_{n}^{(i)}$. Then $\mathbf{Q} \times X \in \mathscr{X}_{n+1}^{(i)}$.

Proof. Since $\mathbf{Q} \times X=\bigcup_{q \in \mathbf{Q}}(\{q\} \times X)$, in all cases $\mathbf{Q} \times X$ is strongly $\sigma-\mathscr{P}_{n}^{(i)}$, hence $\mathscr{P}_{n+1}^{(i)}$ by Lemma 6.2. Let $U \times V$ be a nonempty basic clopen subset of $\mathbf{Q} \times X$.

Case 1: $n=4 k$. Assume $U \times V$ is $\mathscr{P}_{4(k-1)+3}^{2}$. Then by Lemma 6.1, $V$ is $\mathscr{P}_{4(k-1)+3}^{2}$. But $X \in \mathscr{X}_{4 k}$, and by Lemma 6.8, $X$ is nowhere $\mathscr{P}_{4(k-1)+3}^{2}$, contradiction. So $\mathbf{Q} \times X$ is nowhere $\mathscr{P}_{4(k-1)+3}^{2}$, hence so is $U \times V$. Now suppose $U \times V$ is $\mathscr{P}_{4 k}$; then by Lemma 6.8, $U \times V \in \mathscr{X}_{4 k}$, so $U \times V$ is Baire by Lemma 6.7. But $U \times V=$ $\mathrm{U}_{q \in U}(\{q\} \times V)$ is not Baire, contradiction. So $\mathbf{Q} \times X$ is nowhere $\mathscr{P}_{4 k}$.

Case 2: $n=4 k+2, i=1$. If $U \times V$ is $\mathscr{P}_{4 k+1}$, then so is $V$; but $V \in \mathscr{X}_{4 k+2}^{1}$, contradiction. If $U \times V$ is $\mathscr{P}_{4 k+2}^{1}$, then $U \times V$ is $\mathscr{P}_{4 k+2}^{1}$ and nowhere $\mathscr{P}_{4 k+1}$, so $U \times V \in \mathscr{X}_{4 k+2}^{1}$ by Lemma 6.8 and is Baire by Lemma 6.7; hence, as in Case 1, we have a contradiction.

Case 3: $n=4 k+2, i=2$. As above.

As noted in $\S 3$, to obtain elements of $\mathscr{X}_{n}^{(i)}$ for even $n$, we have to consider complements in the Cantor set of spaces belonging to $\mathscr{X}_{m}^{(j)}$ for some odd $m$. Hence the following:

6.10. Lemma. Let $X$ be dense and codense in the Cantor set, and let $k \in \omega$. Then

(a) $X \in \mathscr{X}_{4(k+1)}$ if and only if $C \backslash X$ is $\mathscr{P}_{4 k+3}^{2}$, nowhere $\mathscr{P}_{4 k+2}^{2}$, and nowhere $\mathscr{P}_{4 k+1}$.

(b) $X \in X_{4 k+1}$ if and only if $C \backslash X$ is $\mathscr{P}_{4 k+2}^{2}$, nowhere $\mathscr{P}_{4 k}$, and nowhere $\mathscr{P}_{4(k-1)+3}^{2}$.

Proof. (a) If $X \in \mathscr{X}_{4(k+1)}$, then $X$ is $\mathscr{P}_{4(k+1)}$, so by Lemma 6.4, $C \backslash X$ is $\mathscr{P}_{4 k+3}^{2}$. If $\varnothing \neq V$ is clopen in $C$, and $V \backslash X$ is $\mathscr{P}_{4 k+2}^{2}$, then $\varnothing \neq V \cap X$ is $\mathscr{P}_{4 k+1}$ by Lemma 6.4, contradicting the fact that $X$ is nowhere $\mathscr{P}_{4 k+1}$. Similarly, $V \backslash X$ being $\mathscr{P}_{4 k+1}$ would contradict $X$ being nowhere $\mathscr{P}_{4 k+2}^{2}$. Part (b) and the converse of (a) are proved in the same way. 
6.11. Corollary. Let $X$ be a dense subset of the Cantor set. Then, for each $k \in \omega$ and $i \in\{1,2\}$,

(a) if $X \in \mathscr{X}_{4 k+2}^{i}$, then $C \backslash X \in \mathscr{X}_{4 k+1}$.

(b) if $X \in \mathscr{X}_{4(k-1)+3}^{i}$, then $C \backslash X \in \mathscr{X}_{4 k}$.

Proof. Since in either case, $X$ is nowhere locally compact, $X$ is also codense in $C$. If $X \in \mathscr{X}_{4 k+2}^{1}$, then $X$ is $\mathscr{P}_{4 k+2}^{1}$ and, hence, $\mathscr{P}_{4 k+2}^{2}$; since $X$ is also nowhere $\mathscr{P}_{4 k}$ and nowhere $\mathscr{P}_{4(k-1)+3}^{2}$, Lemma 6.10 applies. The other cases are proved similarly.

6.12. LemMA. For each $n \in \omega$ and $i \in\{1,2\}, \mathscr{X}_{n}^{(i)} \neq \varnothing$.

Proof. This is clear for $n=0$, so assume $\mathscr{X}_{m}^{(j)} \neq \varnothing$ for $m<n(\geqslant 1), j \in\{1,2\}$. If $n$ is odd, then $\mathscr{X}_{n}^{(i)} \neq \varnothing$ by Lemma 6.9, so suppose $n$ is even.

Case 1: $n=4 k$. By the inductive hypothesis $\mathscr{X}_{4(k-1)+3}^{1} \neq \varnothing$, say $X \in \mathscr{X}_{4(k-1)+3}^{1}$. Embed $X$ as a dense subset of $C$; then by Corollary 6.11, $C \backslash X \in \mathscr{X}_{4 k}$.

Case 2: $n=4 k+2, i \in\{1,2\}$. Let $X_{i} \in \mathscr{X}_{4(k-1)+3}^{i}$ and embed $X_{i}$ as a dense subset of the Cantor set; also embed $\mathbf{Q}$ as a dense subset of the Cantor set, and let $Y_{i}=(C \times C) \backslash\left(\mathrm{Q} \times\left(C \backslash X_{i}\right)\right)$. By Corollary 6.11, $C \backslash X_{i} \in \mathscr{X}_{4 k}$; so by Lemma 6.9, $\mathbf{Q} \times\left(C \backslash X_{i}\right) \in \mathscr{X}_{4 k+1}$. Hence, by Lemma 6.10, $Y_{i}$ is $\mathscr{P}_{4 k+2}^{2}$, nowhere $\mathscr{P}_{4 k}$, and nowhere $\mathscr{P}_{4(k-1)+3}^{2}$.

Case 2a: $i=1$. To show that $Y_{1} \in \mathscr{X}_{4 k+2}^{1}$, it suffices to show that $Y_{1}$ is $\mathscr{P}_{4 k+2}^{1}$. But $Y_{1}=\left(\mathbf{Q} \times X_{1}\right) \cup((C \backslash \mathbf{Q}) \times C)$; and since $X_{1}$ is $\mathscr{P}_{4(k-1)+3}^{1}, \mathbf{Q} \times X_{1}$ is also $\mathscr{P}_{4(k-1)+3}^{1}$ by Corollary 6.3. So $Y_{1}$ is $\mathscr{P}_{4(k-1)+3}^{1} \cup$ complete is $\mathscr{P}_{4 k+2}^{1}$.

Case 2b: $i=2$. To show that $Y_{2} \in \mathscr{X}_{4 k+2}^{2}$, it suffices to show that $Y_{2}$ is nowhere $\mathscr{P}_{4 k+2}^{1}$. So suppose $U \times V \neq \varnothing$ is a basic clopen subset of $C \times C$ and $(U \times V) \cap Y_{2}$ is $\mathscr{P}_{4 k+2}^{1}$. Since $\mathbf{Q}$ is dense in $C, U \cap \mathbf{Q} \neq \varnothing$, say $x \in U \cap \mathbf{Q}$; then $(U \times V) \cap Y_{2}$ $\cap(\{x\} \times C)=\{x\} \times\left(V \cap X_{2}\right)$, so $V \cap X_{2}$ is also $\mathscr{P}_{4 k+2}^{1}$; i.e., $V \cap X_{2}=A \cup B$, where $A$ is $\mathscr{P}_{4(k-1)+3}^{1}$ and $B$ is complete. Since $X_{2}$ is nowhere $\mathscr{P}_{4(k-1)+3}^{1}, B$ is dense in $V \cap X_{2}$, so $V \cap X_{2}$ is Baire. This contradicts Lemma 6.7.

We will now state and prove the main theorems of this section.

6.13. THEOREM. Up to homeomorphism each $\mathscr{X}_{n}^{(i)}$ contains exactly one element.

6.14. TheOREM. If $X \in \mathscr{X}_{4 k+2}^{i} \cup \mathscr{X}_{4 k+3}^{i}$ for some $k \in \omega$ and $i \in\{1,2\}$, then $C$ is homogeneous with respect to dense copies of $X$.

6.15. Theorem. Let $A$ be a Borel subset of the Cantor set. For each $k \in \omega$ the following hold:

(a) If $A$ is not $\mathscr{P}_{4 k}$, then $C$ contains a Cantor set $K$ such that $K \cap A \in \mathscr{X}_{4(k-1)+3}^{1}$ and $K \backslash A \in \mathscr{X}_{4 k}$.

(b) If $A$ is not $\mathscr{P}_{4 k+2}^{1}$, then $C$ contains a Cantor set $K$ such that $K \cap A \in \mathscr{X}_{4(k-1)+3}^{2}$ and $K \backslash A \in \mathscr{X}_{4 k}$.

(c) If $A$ is not $\mathscr{P}_{4(k-1)+3}^{2}$, then $C$ contains a Cantor set $K$ such that $K \cap A \in \mathscr{X}_{4 k}$.

(d) If $A$ is not $\mathscr{P}_{4 k+1}$, then $C$ contains a Cantor set $K$ such that $K \cap A \in \mathscr{X}_{4 k+2}^{1}$ and $K \backslash A \in \mathscr{X}_{4 k+1}$.

(e) If $A$ is not $\mathscr{P}_{4 k+3}^{1}$, then $C$ contains a Cantor set $K$ such that $K \cap A \in \mathscr{X}_{4 k+2}^{2}$ and $K \backslash A \in \mathscr{X}_{4 k+1}$. 
We will prove the above theorems by induction on $n$, using the following statements as inductive hypotheses:

(1) Up to homeomorphism $\mathscr{X}_{n}^{(i)}$ contains at most one element.

(2) If $n \in\{4 k+2,4 k+3\}$ for some $k \in \omega, i \in\{1,2\}$, and $X \in \mathscr{X}_{n}^{(i)}$, then $C$ is homogeneous with respect to dense copies of $X$.

(3) If $n=4 k$, then Theorem 6.15(a)-(c) hold for this $k$; if $n=4 k+2$, then Theorem 6.15(d), (e) hold for this $k$.

If (1)-(3) are proved for each $n \in \omega$, then Theorem 6.13 follows from (1) and Lemma 6.12, and Theorems 6.14 and 6.15 are clear from (2) and (3). We have only added Theorem 6.15(c) for convenience: it follows immediately from Theorem 6.15(a) and Lemma 6.4(a).

Since (1) is clear for $n=0$ and (3) follows from Theorem 5.1 for $k=n=0$, we may assume that $(1)-(3)$ have been proved for $m<n(\geqslant 1)$. Note that, since $\left|\mathscr{X}_{m}^{(j)}\right|=1$ for $m<n$, it follows that the unique element $X_{m}^{(j)}$ of $\mathscr{X}_{m}^{(j)}$ is strongly homogeneous: indeed, if $\varnothing \neq V$ is clopen in $X_{m}^{(j)}$, then $V \in \mathscr{X}_{m}^{(j)}$, hence $V \approx X_{m}^{(j)}$.

The proofs of (1)-(3) for $m=n$ consist of a series of lemmas.

\subsection{LEMMA. If $n$ is odd then (1) holds.}

Proof. Let $X \in \mathscr{X}_{n}^{(i)}$; we will show that $X \approx \mathbf{Q} \times Y$, where $Y$ is the unique element of $\mathscr{X}_{n-1}^{(i)}$.

Since $X$ is $\mathscr{P}_{n}^{(i)}$, we can write $X=\bigcup_{j=1}^{\infty} X_{j}$, where $X_{j}$ is closed in $X$ and $\mathscr{P}_{n-1}^{(i)}$ (Lemma 6.2). Fix $j \in \mathbf{N}$ and let $\mathscr{D}$ be a disjoint cover of $X \backslash X_{j}$ by clopen subsets of $X$ such that for each $D \in \mathscr{D}, \operatorname{diam}(D)<d\left(D, X_{j}\right)$.

Claim. If $U$ is open in $X$ and $U \cap X_{j} \neq \varnothing$, then $U \supset D$ for some $D \in \mathscr{D}$.

Indeed, let $x \in B(x, \varepsilon) \subset U$. Since $X$ is nowhere $\mathscr{P}_{n-1}^{(i)}, X_{j}$ is nowhere dense in $X$, so $B(x, \varepsilon / 2) \cap D \neq \varnothing$ for some $D \in \mathscr{D}$. Then $\operatorname{diam}(D)<d\left(D, X_{j}\right)<\frac{1}{2} \varepsilon$ for this $D \in \mathscr{D}$, so $D \subset B(x, \varepsilon) \subset U$.

Case 1: $n=4 k+1$. Since $X$ is nowhere $\mathscr{P}_{4(k-1)+3}^{2}$ and (3) holds for $m=4 k$, we can apply Theorem $6.15(\mathrm{c})$ to each $D \in \mathscr{D}$ and obtain a closed subset $E(D)$ of $D$ such that $E(D) \approx Y \in \mathscr{X}_{4 k}$. Then

$$
A_{j}=X_{j} \cup \bigcup_{D \in \mathscr{D}} E(D)
$$

is closed in $X$, and it is easily seen that $A_{j}$ is $\mathscr{P}_{4 k}$ and, hence, nowhere dense in $X$. If $\varnothing \neq U$ is open in $A_{j}$, say $U=U^{\prime} \cap A_{j}$ with $U^{\prime}$ open in $X$, then either $U^{\prime} \cap X_{j}=\varnothing$ - hence $U \cap E(D) \neq \varnothing$ for some $D \in \mathscr{D}$-or $U^{\prime} \cap X_{j} \neq \varnothing$-hence $U \supset E(D)$ for some $D \in \mathscr{D}$ - by the Claim. In both cases it follows that $U$ is not $\mathscr{P}_{4(k-1)+3}^{2}$ since $E(D)$ is nowhere $\mathscr{P}_{4(k-1)+3}^{2}$, and hence $A_{j} \approx Y$ (Lemma 6.8). Thus $X=\bigcup_{j=1}^{\infty} A_{j}$ satisfies the hypotheses of Theorem 4.3, and hence $X \approx \mathbf{Q} \times Y$.

Case 2: $n=4 k+3$. This is similar to Case 1: since (3) holds for $m=4 k+2$, we here apply Theorem 6.15(d) (if $i=1$ ) or (e) (if $i=2$ ) to obtain $E(D) \in \mathscr{X}_{4 k+2}^{i}$. Then each $A_{j} \approx Y \in \mathscr{X}_{4 k+2}^{i}$, and again $X \approx \mathbf{Q} \times Y$ by Theorem 4.3.

6.17. LEMMA. If $n=4 k+3$ for some $k \in \omega$, then (2) holds. 
Proof. Let $X \in \mathscr{X}_{4 k+2}^{i}(i \in\{1,2\})$. By Lemmas 6.9 and 6.16, $\mathbf{Q} \times X$ is the unique element of $\mathscr{X}_{4 k+3}^{i}$. Since (2) holds for $m=4 k+2$, the Cantor set is homogeneous with respect to dense copies of $X$. Now apply Theorem 4.7.

Lemmas 6.16 and 6.17 show that the inductive hypotheses are satisfied for $m=n$ if $n$ is odd. The remaining lemmas of this section deal with the case where $n$ is even.

6.18. Lemma. Let $k \in \mathbf{N}$ and let $X \in \mathscr{X}_{4 k}$. Then $X$ can be embedded in the Cantor set such that $C \backslash X \in \mathscr{X}_{4(k-1)+3}^{1}$.

Proof. Embed $X$ in $C$ as a dense subset. By Lemma 6.10, $C \backslash X$ is $\mathscr{P}_{4(k-1)+3}^{2}$, nowhere $\mathscr{P}_{4(k-1)+2}^{2}$, and nowhere $\mathscr{P}_{4(k-1)+1}$. Thus we can write $C \backslash X=A \cup B$, where $A$ is $\mathscr{P}_{4(k-1)+1}$ and $B$ is $\sigma$-compact; since $A \backslash B$ is a $G_{\delta}$ subset of $A$, it is $\mathscr{P}_{4(k-1)+1}$ by Lemma 6.1, so we may assume that $A \cap B=\varnothing$. Note that $X \subset C \backslash B$ and $C \backslash B$ is complete. Also, since $C \backslash X$ is nowhere $\mathscr{P}_{4(k-1)+1}, B$ is dense in $C \backslash X$ and, hence, in $C$, so $C \backslash B$ is nowhere locally compact. Hence, by Theorem 2.4 , $C \backslash B \approx \mathbf{P}$. Put $Y=C \backslash B$ and embed $Y$ in $K \approx C$ such that $K \backslash Y \approx \mathbf{Q}$. We claim that $K \backslash X \in \mathscr{X}_{4(k-1)+3}^{1}$.

Indeed, by Lemma 6.10, $K \backslash X$ is nowhere $\mathscr{P}_{4(k-1)+2}^{2}$ and nowhere $\mathscr{P}_{4(k-1)+1}$; and $K \backslash X=(Y \backslash X) \cup \mathbf{Q}=A \cup \mathbf{Q}$ is $\mathscr{P}_{4(k-1)+1} \cup$ countable is $\mathscr{P}_{4(k-1)+3}^{1}$.

6.19. LEMMA. If $n=4 k$ for some $k \in \mathrm{N}$, then (1) holds.

Proof. Let $X_{1}, X_{2} \in \mathscr{X}_{4 k}$. By Lemma 6.18, $X_{1}$ and $X_{2}$ can be embedded in $C$ such that $C \backslash X_{1}, C \backslash X_{2} \in \mathscr{X}_{4(k-1)+3}^{1}$. Since (1) holds for $m=4(k-1)+3, C \backslash X_{1} \approx$ $C \backslash X_{2}$, and, by (2), there exists a homeomorphism $h: C \rightarrow C$ such that $h\left[C \backslash X_{1}\right]=$ $C \backslash X_{2}$, and thus $h\left[X_{1}\right]=X_{2}$.

6.20. Lemma. Suppose $n=4 k$ for some $k \in \mathbf{N}$, and let $A$ be a Borel subset of $C$.

(a) If $A$ is not $\mathscr{P}_{4 k}$, then $C$ contains a Cantor set $K$ such that $K \cap A \in \mathscr{X}_{4(k-1)+3}^{1}$ and $K \backslash A \in \mathscr{X}_{4 k}$.

(b) If $A$ is not $\mathscr{P}_{4 k+2}^{1}$, then $C$ contains a Cantor set $K$ such that $K \cap A \in \mathscr{X}_{4(k-1)+3}^{2}$ and $K \backslash A \in \mathscr{X}_{4 k}$.

Proof. (a) Let $\mathscr{P}=\mathscr{P}_{4(k-1)+1}$ and let $Z$ be the unique element of $\mathscr{X}_{4(k-1)+2}^{1}$; we will show that the hypotheses of Lemma 5.2 are satisfied. By Lemma $6.1, \mathscr{P}$ is closed-hereditary; by Corollary $6.3 \mathscr{P}$ is strongly $\sigma$-additive. Let $B$ be a Borel subset of the Cantor set which is not $\mathscr{P}$; since (3) holds for $m=4(k-1)+2, C$ contains a Cantor set $K$ such that $K \cap B \in \mathscr{X}_{4(k-1)+2}^{1}$ and $K \backslash B \in \mathscr{X}_{4(k-1)+1}$. Then $K \cap B \approx Z$, and by Lemma 6.2 we can write $K \backslash B=\bigcup_{i=1}^{\infty} X_{i}$, where $X_{i}$ is closed in $K \backslash B$ and $\mathscr{P}_{4(k-1)}$. Put $G=\bigcup_{i=1}^{\infty} \bar{X}_{i}$; then $G$ is $\sigma$-compact, and since $\bar{X}_{i} \cap B=\bar{X}_{i} \backslash X_{i}$ is $\mathscr{P}_{4(k-2)+3}^{2}$ by Lemma 6.4, $G \cap B$ is strongly $\sigma-\mathscr{P}_{4(k-2)+3}^{2}$, hence $\mathscr{P}_{4(k-2)+3}^{2}$ by Corollary 6.3; thus $G \cap B$ is $\mathscr{P}$ by Lemma 6.5. So by Lemma 5.2, if $A$ is a Borel subset of $C$ which is not $\mathscr{P}_{4 k}$, or equivalently, not $\mathscr{P} \cup$ complete, then $C$ contains a Cantor set $K^{\prime}$ such that $K^{\prime} \cap A \approx \mathbf{Q} \times Z$. Put $K=\overline{K^{\prime} \cap A}$; then $K \approx C, K \cap A \in \mathscr{X}_{4(k-1)+3}^{1}$ by Lemma 6.9, and hence $K \backslash A \in \mathscr{X}_{4 k}$ by Corollary 6.11.

(b) Since $\mathscr{P}_{4 k+2}^{1} \equiv \mathscr{P}_{4(k-1)+3}^{1} \cup$ complete, here we put $\mathscr{P}=\mathscr{P}_{4(k-1)+3}^{1}$; then $\mathscr{P}$ is again closed-hereditary and strongly $\sigma$-additive. Let $Z$ be the unique element of 
$\mathscr{X}_{4(k-1)+2}^{2}$, and suppose $B$ is a Borel subset of the Cantor set which is not $\mathscr{P}$; since (3) holds for $m=4(k-1)+2, C$ contains a Cantor set $K$ such that $K \cap B \in$ $\mathscr{X}_{4(k-1)+2}^{2}$ and $K \backslash B \in \mathscr{X}_{4(k-1)+1}$. As above, we put $K \backslash B=\bigcup_{i=1}^{\infty} X_{i}$ and $G=$ $\cup_{i=1}^{\infty} \bar{X}_{i}$; then $G \cap B$ is $\mathscr{P}_{4(k-2)+3}^{2}$; hence, $G \cap B$ is $\mathscr{P}$ by Lemma 6.5. So by Lemma 5.2, if $A$ is a Borel subset of $C$ which is not $\mathscr{P}_{4 k+2}^{1}$, then $C$ contains a Cantor set $K^{\prime}$ such that $K^{\prime} \cap A \approx \mathbf{Q} \times Z$. Put $K=\overline{K^{\prime} \cap A}$; then $K \approx C, K \cap A \in \mathscr{X}_{4(k-1)+3}^{2}$ by Lemma 6.9; hence, $K \backslash A \in \mathscr{X}_{4 k}$ by Corollary 6.11.

Lemmas 6.18-6.20 show that the inductive hypotheses are satisfied if $n=4 k$ for some $k \in \mathbf{N}$.

6.21. Lemma. Let $k \in \omega, i \in\{1,2\}$, and let $X \in \mathscr{X}_{4 k+2}^{i}$ be a dense subset of the Cantor set. Then there exist closed nowhere dense subspaces $A_{j}$ of $C \backslash X$ such that $C \backslash X=\cup_{j=1}^{\infty} A_{j}$ and $\bar{A}_{j} \backslash A_{j} \in \mathscr{X}_{4(k-1)+3}^{i}$ is dense in $\bar{A}_{j}$.

Proof. By Corollary 6.11, $C \backslash X \in \mathscr{X}_{4 k+1}$; so by Lemma 6.2 we can write $C \backslash X=\cup_{l=1}^{\infty} X_{l}$, with $X_{l}$ closed in $C \backslash X$ and $\mathscr{P}_{4 k}$. Since $\bar{X}_{l} \backslash X_{l}=\bar{X}_{l} \cap X$ is closed in $X$, it is $\mathscr{P}_{4 k+2}^{i}$ by Lemma 6.1; hence, $\bar{X}_{l} \backslash X_{l}=G_{l} \cup H_{l}$, where $G_{l}$ is complete and $H_{l}$ is $\mathscr{P}_{4(k-1)+3}^{i}$. Then $\bar{X}_{l} \backslash G_{l}$ is $\sigma$-compact, say

$$
\bar{X}_{l} \backslash G_{l}=\bigcup_{m=1}^{\infty} K(l, m),
$$

with $K(l, m)$ compact. Since $\{K(l, m) \backslash X: l, m \in \mathbf{N}\}$ is countable, we can enumerate the nonempty elements of this set as $\left\{M_{j}: j \in \mathbf{N}\right\}$. Fix $j \in \mathbf{N}$. Since $M_{j}$ is a closed subset of some $X_{l}, M_{j}$ is $\mathscr{P}_{4 k}$, and $\bar{M}_{j} \cap X=\bar{M}_{j} \cap H_{l}$ for this same $l$; hence, $\bar{M}_{j} \cap X$ is $\mathscr{P}_{4(k-1)+3}^{i}$.

Now let $\mathscr{D}$ be a disjoint cover of $C \backslash \bar{M}_{j}$ by clopen subsets of $C$ such that for each $D \in \mathscr{D}, \operatorname{diam}(D)<d\left(D, \bar{M}_{j}\right)$. Since $X$ is nowhere $\mathscr{P}_{4 k}$ (if $\left.i=1\right)$, resp. nowhere $\mathscr{P}_{4 k+2}^{1}$ (if $i=2$ ), and since (3) holds for $m=4 k$, each $D \in \mathscr{D}$ contains a Cantor set $K(D)$ such that $K(D) \cap X \in \mathscr{X}_{4(k-1)+3}^{i}$ and $K(D) \backslash X \in \mathscr{X}_{4 k}$. Put

$$
K_{j}=\bar{M}_{j} \cup \bigcup_{D \in \mathscr{D}} K(D)
$$

and $A_{j}=K_{j} \backslash X$; we claim that the sets $A_{j}$ constructed this way are as required.

First note that $C \backslash X=\bigcup_{j=1}^{\infty} A_{j}$ since $K_{j} \backslash X \supset M_{j}$. Furthermore, since $C \backslash X$ is nowhere $\mathscr{P}_{4 k}, M_{j}$ is nowhere dense in $C \backslash X$, and hence $\bar{M}_{j}$ is nowhere dense in $C$; since $\operatorname{diam}(D)<d\left(D, \bar{M}_{j}\right)$ for each $D \in \mathscr{D}$, it then follows that $\overline{\cup_{D \in \mathscr{D}} K(D)}=K_{j}$; thus

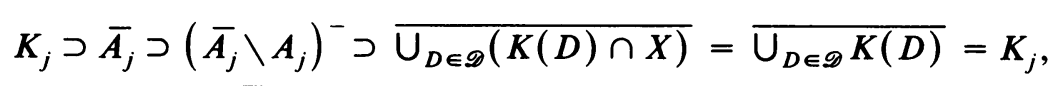

so $\overline{A_{j}} \backslash A_{j}$ is dense in $\overline{A_{j}}$. It therefore remains to be shown that $\bar{A}_{j} \backslash A_{j} \in \mathscr{X}_{4(k-1)+3}^{i}$. Clearly,

$$
\overline{A_{j}} \backslash A_{j}=\left(\bar{M}_{j} \cap X\right) \cup \bigcup_{D \in \mathscr{D}}(K(D) \cap X)
$$

is strongly $\sigma-\mathscr{P}_{4(k-1)+3}^{i}$ is $\mathscr{P}_{4(k-1)+3}^{i}$ by Corollary 6.3, and, as in the proof of Lemma 6.16, it follows that if $\varnothing \neq U$ is open in $\overline{A_{j}} \backslash A_{j}$, then $U \cap K(D) \cap X \neq \varnothing$ for some $D \in \mathscr{D}$; hence, since $K(D) \cap X \in \mathscr{X}_{4(k-1)+3}^{i}, U$ is not $\mathscr{P}_{4(k-1)+2}^{2}$ and not $\mathscr{P}_{4(k-1)+1}$ (if $i=1$ ), respectively, not $\mathscr{P}_{4(k-1)+3}^{1}$ (if $i=2$ ). Thus, $\overline{A_{j}} \backslash A_{j} \in \mathscr{X}_{4(k-1)+3}^{i}$. Hence, 
$A_{j} \in \mathscr{X}_{4 k}$ by Corollary 6.11, and hence $A_{j}$ is nowhere dense in $C \backslash X$ since $C \backslash X$ is nowhere $\mathscr{P}_{4 k}$.

6.22. LeMmA. If $n=4 k+2$ for some $k \in \omega, i \in\{1,2\}$, and $X_{1}, X_{2} \in \mathscr{X}_{n}^{i}$ are dense subsets of $C$, then there exists a homeomorphism $h: C \rightarrow C$ such that $h\left[X_{1}\right]=X_{2}$. Thus, (1) and (2) are satisfied if $n=4 k+2$.

Proof. Let $A$ be the unique element of $\mathscr{X}_{4(k-1)+3}^{i}$. By Lemma 6.21 we can write $C \backslash X_{1}=\bigcup_{j=1}^{\infty} A_{j}, C \backslash X_{2}=\bigcup_{j=1}^{\infty} B_{j}$ such that $\bar{A}_{j} \backslash A_{j} \approx \bar{B}_{j} \backslash B_{j} \approx A, \bar{A}_{j} \backslash A_{j}$ is dense in $\bar{A}_{j}, \bar{B}_{j} \backslash B_{j}$ is dense in $\bar{B}_{j}, A_{j}$ is closed and nowhere dense in $C \backslash X_{1}$, and $B_{j}$ is closed and nowhere dense in $C \backslash X_{2}$. Hence, the required homeomorphism can be obtained from Lemma 4.6.

6.23. Lemma. Suppose $n=4 k+2$ for some $k \in \mathbf{N}$, and let $A$ be a Borel subset of C.

(a) If $A$ is not $\mathscr{P}_{4 k+1}$, then $C$ contains a Cantor set $K$ such that $K \cap A \in \mathscr{X}_{4 k+2}^{1}$ and $K \backslash A \in \mathscr{X}_{4 k+1}$.

(b) If $A$ is not $\mathscr{P}_{4 k+3}^{1}$, then $C$ contains a Cantor set $K$ such that $K \cap A \in \mathscr{X}_{4 k+2}^{2}$ and $K \backslash A \in \mathscr{X}_{4 k+1}$.

Proof. (a) Let $\mathscr{P}=\mathscr{P}_{4 k+1}$, let $Z$ be the unique element of $\mathscr{X}_{4 k}$, and let $Y$ be the unique element of $\mathscr{X}_{4(k-1)+3}^{1}$. By Lemma $6.1, \mathscr{P}$ is closed-hereditary; by Corollary $6.3, \mathscr{P}$ is strongly $\sigma$-additive. Let $B$ be a Borel subset of the Cantor set which is not $\mathscr{P}$. Then $B$ is not $\mathscr{P}_{4 k}$ by Lemma 6.5; hence, since (3) holds for $m=4 k, C$ contains a Cantor set $K$ such that $K \cap B \in \mathscr{X}_{4(k-1)+3}^{1}$ and $K \backslash B \in \mathscr{X}_{4 k}$; thus $K \cap B \approx Y$, $K \backslash B \approx Z$, and $K \cap B$ is $\mathscr{P}$ by Lemma 6.5. Hence, by Lemma 5.3, if $A$ is a Borel subset of the Cantor set which is not $\mathscr{P}$, then $C$ contains Cantor sets $B_{i}$, for $i \in \omega$, such that $K=\left(\cup_{i=0}^{\infty} B_{i}\right)^{-} \approx C$ and, for each $i \in \omega$,

(i) $B_{i}$ is nowhere dense in $K$;

(ii) $K \backslash A=\cup_{i=0}^{\infty}\left(B_{i} \backslash A\right)$;

(iii) $B_{0} \backslash A \approx Z \approx\left(B_{i+1} \backslash B_{i}\right) \backslash A$;

(iv) $B_{0} \cap A \approx Y \approx\left(B_{i+1} \backslash B_{i}\right) \cap A$.

Since $B_{i+1} \backslash B_{i}$ is open in $B_{i+1}$, we can write

$$
B_{i+1} \backslash B_{i}=\bigcup_{j=0}^{\infty} K_{j}^{i}
$$

where $K_{j}^{i}$ is clopen in $B_{i+1}$. Since both $Y$ and $Z$ are strongly homogeneous, we have $K_{j}^{i} \cap A \approx Y$ and $K_{j}^{i} \backslash A \approx Z$. Hence,

$$
K \backslash A=\left(B_{0} \backslash A\right) \cup \bigcup_{i, j=0}^{\infty}\left(K_{j}^{i} \backslash A\right) \approx \mathbf{Q} \times Z
$$

by (i) and Theorem 4.3 , so $K \backslash A \in \mathscr{X}_{4 k+1}$ by Lemma 6.9. Then $K \cap A$ is nowhere $\mathscr{P}_{4 k}$ and nowhere $\mathscr{P}_{4(k-1)+3}^{2}$ by Lemma 6.10. So we only have to show that $K \cap A$ is $\mathscr{P}_{4 k+2}^{1}$. However, by (ii),

$$
K \cap A=\left(B_{0} \cap A\right) \cup \bigcup_{i, j=0}^{\infty}\left(K_{j}^{i} \cap A\right) \cup K \backslash \bigcup_{i=0}^{\infty} \bar{B}_{i}
$$

is strongly $\sigma-\mathscr{P}_{4(k-1)+3}^{1} \cup$ complete is $\mathscr{P}_{4(k-1)+3}^{1} \cup$ complete is $\mathscr{P}_{4 k+2}^{1}$. 
(b) Let $\mathscr{P}=\mathscr{P}_{4 k+3}^{1}$, let $Z$ be the unique element of $\mathscr{X}_{4 k}$, and let $Y$ be the unique element of $\mathscr{X}_{4(k-1)+3}^{2}$. Then $\mathscr{P}^{2}$ is closed-hereditary and strongly $\sigma$-additive. Let $B$ be a Borel subset of the Cantor set which is not $\mathscr{P}$. Then $B$ is not $\mathscr{P}_{4 k+2}^{1}$, so since (3) holds for $m=4 k, C$ contains a Cantor set $K$ such that $K \cap B \approx Y, K \backslash B \approx Z$, and $K \cap B$ is $\mathscr{P}$ by Lemma 6.5. So again by Lemma 5.3, we find Cantor sets $B_{i}$ as under (a); then $K \backslash A \in \mathscr{X}_{4 k+1}$; hence, by Lemma $6.10, K \cap A$ is $\mathscr{P}_{4 k+2}^{2}$ and nowhere $\mathscr{P}_{4(k-1)+3}^{2}$, so it suffices to show that $K \cap A$ is nowhere $\mathscr{P}_{4 k+2}^{1}$. Put $B_{i+1} \backslash B_{i}=$ $\cup_{j=0}^{\infty} K_{j}^{i}$ as in (a), and note that if $\varnothing \neq U$ is open in $K \cap A$, then $U \cap K_{j}^{i} \neq \varnothing$ for some $i, j \in \omega$; thus, $U$ contains a closed copy of $Y$. Now suppose $U$ is $\mathscr{P}_{4 k+2}^{1}$; then so is $Y$ by the preceding remark; i.e., $Y=A \cup B$, where $A$ is $\mathscr{P}_{4(k-1)+3}^{1}$ and $B$ is complete. Since $Y \in \mathscr{X}_{4(k-1)+3}^{2}, Y$ is nowhere $\mathscr{P}_{4(k-1)+3}^{1}$; hence, $B$ is dense in $Y$, so $Y$ is Baire, contradicting Lemma 6.7.

This completes the proof of Theorems 6.13-6.15. In $\S 8$ we will show that there are no homogeneous (non- $\sigma$-compact) $\Sigma_{n}$-subsets of the Cantor set other than those defined and characterized above.

6.24. REMARK. Using a technique similar to that of [6], it can be shown that Theorem 6.15 also holds for Borel sets in arbitrary compact spaces (see [5]). Also, the converse of Theorem 6.15 is true; e.g., if $A$ is a Borel subset of the Cantor set which contains a closed copy of $X \in \mathscr{X}_{4(k-1)+3}^{1}$, then $A$ is not $\mathscr{P}_{4 k}$, etc.

We conclude this section with the following theorem, announced in $\S 3$.

6.25. THEOREM. If we order the properties $\mathscr{P}_{n}^{(i)}$ by $\mathscr{P}_{4 k}<\mathscr{P}_{4 k+1}<\mathscr{P}_{4 k+2}^{1}<\mathscr{P}_{4 k+3}^{1}$ $<\mathscr{P}_{4 k+2}^{2}<\mathscr{P}_{4 k+3}^{2}<\mathscr{P}_{4(k+1)}$, then for each $i \in\{1,2\}$ and each $n \in \omega$, we have $X \in \mathscr{X}_{n}^{(i)}$ if and only if $X$ is $\mathscr{P}_{n}^{(i)}$ and nowhere $\mathscr{P}_{m}^{(j)}$ for $\mathscr{P}_{m}^{(j)}<\mathscr{P}_{n}^{(i)}$.

Proof. Apply Lemma 6.5.

6.26. CoROllaRY. If $\mathscr{P}_{m}^{(j)}<\mathscr{P}_{n}^{(i)}$, then $\mathscr{X}_{m}^{(j)} \cap \mathscr{X}_{n}^{(i)}=\varnothing$; thus, all spaces characterized in this section are topologically distinct.

7. Spaces which are $\mathscr{P}_{\beta}$ for $\beta \geqslant \omega$. We recall the topological properties $\mathscr{P}_{\beta}(\beta \geqslant \omega)$ described in Definition 3.6:

Let $\alpha \in\left[\omega, \omega_{1}\right)$ be a limit ordinal, and let $n \in \omega$. Then

$X$ is $\mathscr{P}_{\alpha+2 n}$ if and only if $X=\bigcup_{i=1}^{\infty} A_{i} \cup B$, where $B$ is $\Sigma_{n}, A_{i}$ is closed in $X$, and $A_{i}$ is $\mathscr{P}_{\gamma_{i}}$ for some $\gamma_{i}<\alpha$;

$X$ is $\mathscr{P}_{\alpha+2 n+1}$ if and only if $X$ is $\mathscr{P}_{\alpha+2 n} \cup$ complete.

Here, " $X$ is $\mathscr{P}_{\gamma}$ for some $\gamma<\omega$ " means that $X$ is $\mathscr{P}_{4 k}$ or $\mathscr{P}_{4 k+1}$ for some $k \in \omega$. Of course, " $X$ is $\mathscr{P}_{\omega}$ " is equivalent to " $X=\cup_{i=1}^{\infty} X_{i}$, where $X_{i}$ is closed in $X$ and $\mathscr{P}_{n_{i}}^{\left(j_{i}\right)}$ for some $n_{i} \in \omega, j_{i} \in\{1,2\}$ " (Lemma 6.5).

7.1. LEMMA. For each $\beta \in\left[\omega, \omega_{1}\right), \mathscr{P}_{\beta}$ is hereditary with respect to $G_{\delta}$-subsets.

Proof. For $\beta=\omega$ this follows from the fact that $\mathscr{P}_{4 k}$ and $\mathscr{P}_{4 k+1}$ are hereditary with respect to $G_{\delta}$-subsets (Lemma 6.1); proceed inductively. 
7.2. Lemma. Let $X$ be a topological space, let $\alpha \in\left[\omega, \omega_{1}\right)$ be a limit ordinal, and let $n \in \omega$. Then

(a) $X$ is $\mathscr{P}_{\alpha+2 n}$ if and only if $X$ is $\mathscr{P}_{\alpha} \cup \Sigma_{n}$.

(b) $X$ is $\mathscr{P}_{\alpha+2 n+1}$ if and only if $X=\bigcup_{i=1}^{\infty} A_{i} \cup B \cup G$, where $A_{i}$ is $P_{\beta_{i}}$ for some $\beta_{i}<\alpha, B$ is $\Sigma_{n}, G$ is complete, and $A_{i}$ is closed in $X$.

Proof. (a) If $X$ is $\mathscr{P}_{\alpha+2 n}$, then clearly $X$ is $\mathscr{P}_{\alpha} \cup \Sigma_{n}$. We will prove the converse by induction. Suppose $X=\cup_{i=1}^{\infty} A_{i} \cup B$, where $A_{i}$ is closed in $\bigcup_{i=1}^{\infty} A_{i}, A_{i}$ is $\mathscr{P}_{\beta_{i}}$ for some $\beta_{i}<\alpha$, and $B$ is $\Sigma_{n}$, and first consider the case $\alpha=\omega$. Then $\overline{A_{i}}=A_{i} \cup\left(\overline{A_{i}} \cap B\right)$ is $\mathscr{P}_{\beta_{i}} \cup \Sigma_{n}$; hence, $\bar{A}_{i}$ is $\mathscr{P}_{\gamma_{i}}$ for some $\gamma_{i}<\omega$, so $X=\bigcup_{i=1}^{\infty} \bar{A}_{i} \cup B$ is $\mathscr{P}_{\omega+2 n}$. Now assume we are done for limit ordinals $\delta<\alpha(>\omega)$; then we may assume that for each $i, \beta_{i} \geqslant \omega$, and hence $\beta_{i}=\delta_{i}+n_{i}$ for some limit ordinal $\delta_{i} \in[\omega, \alpha)$ and some $n_{i} \in \omega$. If $n_{i}$ is even, then $\overline{A_{i}}$ is $\mathscr{P}_{\delta_{i}+n_{i}} \cup \Sigma_{n}$ is $\mathscr{P}_{\delta_{i}} \cup \Sigma_{n_{i} / 2} \cup \Sigma_{n}$ is $\mathscr{P}_{\delta_{i}+\left(n_{i} / 2+n\right)}$ by the inductive hypothesis, and if $n$ is odd, then $\overline{A_{i}}$ is $\mathscr{P}_{\delta_{i}+n_{i}-1} \cup$ complete $\cup \Sigma_{n}$, hence $\mathscr{P}_{\delta_{i}+n_{i}-1} \cup \Sigma_{n+1}$ is $\mathscr{P}_{\delta_{i}+\left(n_{i}-1+(n+1) / 2\right)}$ by the inductive hypothesis. So each $\overline{A_{i}}$ is $\mathscr{P}_{\gamma_{i}}$ for some $\gamma_{i}<\alpha$, and thus $X=\bigcup_{i=1}^{\infty} \bar{A}_{i} \cup B$ is $\mathscr{P}_{\alpha+2 n}$.

(b) The "if" part is clear, so suppose $X=A \cup G$, where $A$ is $\mathscr{P}_{\alpha+2 n}$ and $G$ is complete. Put

$$
A=\bigcup_{i=1}^{\infty} B_{i} \cup B
$$

where $B_{i}$ is closed in $A$ and $\mathscr{P}_{\beta_{i}}$ for some $\beta_{i}<\alpha$, and $B$ is $\Sigma_{n}$, and let

$$
A_{i}=\bar{B}_{i}=B_{i} \cup\left(\bar{B}_{i} \cap G\right) \text {. }
$$

Then $A_{i}$ is $\mathscr{P}_{\beta_{i}} \cup$ complete. Hence, if $\beta_{i}<\omega$, then $A_{i}$ is $\mathscr{P}_{\beta_{i}+4}$; if $\beta_{i} \geqslant \omega$ is even, then $A_{i}$ is $\mathscr{P}_{\beta_{i}+1}$; and if $\beta_{i} \geqslant \omega$ is odd, then $A_{i}$ is $\mathscr{P}_{\beta_{i}}$. In all cases, $A_{i}$ is $\mathscr{P}_{\gamma_{i}}$ for some $\gamma_{i}<\alpha$, and $A_{i}$ is closed in $X$. Hence, $X=\bigcup_{i=1}^{\infty} A_{i} \cup B \cup G$ is as required.

7.3. CorollaRY. If $\beta \in\left[\omega, \omega_{1}\right)$, then $\mathscr{P}_{\omega} \rightarrow \mathscr{P}_{\beta} \rightarrow \mathscr{P}_{\beta+1}$.

7.4. LEMMA. Let $X$ be a topological space, let $\alpha \in\left[\omega, \omega_{1}\right)$ be a limit ordinal, and let $n \in \mathbf{N}$. Then $X$ is $\mathscr{P}_{\alpha+2 n}$ if and only if $X$ is strongly $\sigma-\mathscr{P}_{\alpha+2 n-1}$.

Proof. Suppose $X$ is $\mathscr{P}_{\alpha+2 n}$, say $X=\cup_{i=1}^{\infty} A_{i} \cup B$, where $B$ is $\Sigma_{n}$ and $A_{i}$ is closed in $X$ and $\mathscr{P}_{\beta_{i}}$ for some $\beta_{i}<\alpha$. Then $A_{i}$ is also $\mathscr{P}_{\alpha} \cup \Sigma_{n-1} \cup$ complete. By Lemma $6.2, B$ is strongly $\sigma-\left(\sum_{n-1} \cup\right.$ complete), say $B=\bigcup_{i=1}^{\infty} B_{i}$, where $B_{i}$ is $\Sigma_{n-1} \cup$ complete and closed in $B$; so by Lemma 7.1, $\bar{B}_{i}=B_{i} \cup\left(\bar{B}_{i} \cap A\right)$ is $\Sigma_{n-1} \cup$ complete $\cup \mathscr{P}_{\alpha}$, and thus $X=\cup_{i=1}^{\infty} A_{i} \cup \cup_{i=1}^{\infty} \bar{B}_{i}$ is strongly $\sigma-\left(\mathscr{P}_{\alpha} \cup \Sigma_{n-1} \cup\right.$ complete $)$ is strongly $\sigma-\mathscr{P}_{\alpha+2 n-1}$ by Lemma 7.2. Conversely, assume that $X=\bigcup_{i=1}^{\infty} X_{i}$, where $X_{i}$ is closed in $X$ and $\mathscr{P}_{\alpha+2 n-1}$; then by Lemma 7.2, we can put

$$
X_{i}=\bigcup_{j=1}^{\infty} A_{i}^{j} \cup B_{i},
$$


where $A_{i}^{j}$ is closed in $X_{i}$, hence in $X, B_{i}$ is $\Sigma_{n-1} \cup$ complete, and $A_{i}^{j}$ is $\mathscr{P}_{\beta(i, j)}$ for some $\beta(i, j)<\alpha$. Then

$$
A=\bigcup_{i, j=1}^{\infty} A_{i}^{j}
$$

is an $F_{\sigma}$ in $X$, so $B_{i} \backslash A$ is $\Sigma_{n-1} \cup$ complete; and if

$$
x \in \overline{B_{i} \backslash A} \cap \bigcup_{j=1}^{\infty}\left(B_{j} \backslash A\right),
$$

then $x \in \bar{B}_{i} \subset \bar{X}_{i}=X_{i}$, so $x \in X_{i} \backslash A \subset B_{i} \backslash A$. So $\cup_{i=1}^{\infty}\left(B_{i} \backslash A\right)$ is strongly $\sigma-\left(\Sigma_{n-1} \cup\right.$ complete $)$ is $\Sigma_{n}$ by Lemma 6.2; hence,

$$
A=\bigcup_{i, j=1}^{\infty} A_{i}^{j} \cup \bigcup_{i=1}^{\infty}\left(B_{i} \backslash A\right)
$$

is $\mathscr{P}_{\alpha+2 n}$.

7.5. CoROllaRy. For even $\beta \in\left[\omega, \omega_{1}\right), \mathscr{P}_{\beta}$ is a stongly $\sigma$-additive property.

7.6. Lemma. Let $X$ be compact, and let $A$ be a subset of $X$. If $A$ is $\mathscr{P}_{\beta}$ for some $\beta$ $\in\left[\omega, \omega_{1}\right)$, then $X \backslash A$ is $\mathscr{P}_{\beta+1}$.

Proof. First note that if $A$ is $\Sigma_{n}$ or, equivalently, $\mathscr{P}_{4(n-1)+1}$, then $X \backslash A$ is $\mathscr{P}_{4(n-1)+2}^{2}$ by Lemma 6.4 , hence, by Lemma $6.5, X \backslash A$ is $\mathscr{P}_{4 n}$ is $\Sigma_{n} \cup$ complete (clearly, this is also true for $n=0$ ). Similarly, if $A$ is $\Sigma_{n} \cup$ complete, then $X \backslash A$ is $\Sigma_{n+1}$.

We prove the lemma by induction. If $\beta=\omega$ and $A$ is $\mathscr{P}_{\beta}$, then $A=\bigcup_{i=1}^{\infty} A_{i}$, where $A_{i}$ is closed in $A$ and $\Sigma_{n_{i}}$ for some $n_{i} \in \omega$; hence,

$$
X \backslash A=\bigcup_{i=1}^{\infty}\left(\overline{A_{i}} \backslash A_{i}\right) \cup X \backslash \bigcup_{i=1}^{\infty} \overline{A_{i}} .
$$

Now $\overline{A_{i}} \backslash A_{i}$ is $\Sigma_{n_{i}} \cup$ complete by the above remark and closed in $X \backslash A$; and $X \backslash \cup_{i=1}^{\infty} \bar{A}_{i}$ is complete. So $X \backslash A$ is $\mathscr{P}_{\omega} \cup$ complete is $\mathscr{P}_{\omega+1}$. Thus we can assume that the lemma is true for $\gamma<\beta(>\omega)$; let $\beta=\alpha+n$, where $\alpha \in\left[\omega, \omega_{1}\right)$ is a limit ordinal. Put $A=\cup_{i=1}^{\infty} A_{i} \cup B$, where $A_{i}$ is closed in $A$ and $\mathscr{P}_{\beta_{i}}$ for some $\beta_{i} \in[\omega, \alpha)$, and $B$ is $\Sigma_{n / 2}$ (if $n$ is even) or $\Sigma_{(n-1) / 2} \cup$ complete (if $n$ is odd). Then

$$
X \backslash A=\bigcup_{i=1}^{\infty}\left(\overline{A_{i} \backslash A_{i}}\right) \cup(X \backslash B) \backslash \bigcup_{i=1}^{\infty} \overline{A_{i}} .
$$

By the inductive hypothesis, $\overline{A_{i}} \backslash A_{i}$ is $\mathscr{P}_{\gamma_{i}}$ for $\gamma_{i}=\beta_{i}+1 \in[\omega, \alpha)$, and $X \backslash B$ is $\Sigma_{n / 2} \cup$ complete (if $n$ is even) or $\Sigma_{(n+1) / 2}$ (if $n$ is odd) by the above remark; hence, the same holds for the $G_{\delta}$-subset $(X \backslash B) \backslash \bigcup_{i=1}^{\infty} \bar{A}_{i}$ of $X \backslash B$. Hence, $X \backslash A$ is $\mathscr{P}_{\alpha+n+1}$ is $\mathscr{P}_{\beta+1}$.

For each pair $(\beta, i)$, with $\beta \in\left[\omega, \omega_{1}\right)$ and $i=2$ if $\beta$ is a limit (respectively, $i \in\{1,2\}$ if $\beta$ is a successor), we now define a class of spaces $\mathscr{X}_{\beta}^{i}$ and show that, up to homeomorphism, each $\mathscr{X}_{\beta}^{i}$ contains exactly one element. 
7.7. Definition. Let $X$ be zero-dimensional, let $\alpha \in\left[\omega, \omega_{1}\right)$ be a limit ordinal, and let $n \in \omega ;$ then

$X \in \mathscr{X}_{\alpha}^{2}$ if and only if $X$ is $\mathscr{P}_{\alpha}$ and nowhere $\mathscr{P}_{\beta}$ for each $\beta<\alpha$;

$X \in \mathscr{X}_{\alpha+2 n+1}^{1}$ if and only if $X$ is $\mathscr{P}_{\alpha+2 n+1}$, nowhere $\mathscr{P}_{\alpha+2 n}$, and does not contain any closed subsets belonging to $\mathscr{X}_{\alpha+2 n}^{2}$;

$X \in \mathscr{X}_{\alpha+2 n+1}^{2}$ if and only if $X$ is $\mathscr{P}_{\alpha+2 n+1}$, nowhere $\mathscr{P}_{\alpha+2 n}$, and every nonempty clopen subset of $X$ contains a closed subset belonging to $\mathscr{X}_{\alpha+2 n}^{2}$;

$X \in \mathscr{X}_{\alpha+2 n+2}^{1}$ if and only if $X$ is $\mathscr{P}_{\alpha+2 n+2}$, nowhere $\mathscr{P}_{\alpha+2 n+1}$, and does not contain any closed subsets belonging to $\mathscr{X}_{\alpha+2 n+1}^{2}$;

$X \in \mathscr{X}_{\alpha+2 n+2}^{2}$ if and only if $X$ is $\mathscr{P}_{\alpha+2 n+2}$, nowhere $\mathscr{P}_{\alpha+2 n+1}$, and every nonempty clopen subset of $X$ contains a closed subset belonging to $\mathscr{X}_{\alpha+2 n+1}^{2}$.

7.8. Lemma. Let $\beta \in\left[\omega, \omega_{1}\right), i \in\{1,2\}$, and $X \in \mathscr{X}_{\beta}^{i}$.

(a) If $\beta$ is even, then $X$ is not Baire.

(b) If $\beta$ is odd, then $X$ contains a dense complete subset; hence $X$ is Baire.

Proof. (a) By the definition of $\mathscr{P}_{\beta}$ (if $\beta$ is a limit) respectively by Lemma 7.4 (if $\beta$ is a successor), we can write $X=\bigcup_{i=1}^{\infty} X_{i}$, where $X_{i}$ is closed in $X$, and $\mathscr{P}_{\beta_{i}}$ for some $\beta_{i}<\beta$ (respectively $\mathscr{P}_{\beta-1}$ ). Since $X$ is nowhere $\mathscr{P}_{\beta_{i}}\left(\right.$ respectively, nowhere $\mathscr{P}_{\beta-1}$ ), each $X_{i}$ is nowhere dense in $X$.

(b) Write $X=A \cup B$, where $A$ is $\mathscr{P}_{\beta-1}$ and $B$ is complete; since $X$ is nowhere $\mathscr{P}_{\beta-1}, B$ is dense in $X$.

We now state and prove the main theorems of this section.

7.9. TheOREM. For each $\beta \in\left[\omega, \omega_{1}\right), i \in\{1,2\}$ (respectively, $i=2$ if $\left.\lim (\beta)\right), \mathscr{X}_{\beta}^{i}$ contains exactly one element up to homeomorphism.

7.10. TheOREM. If $X \in \mathscr{X}_{\beta}^{i}$ for some $\beta \in\left[\omega, \omega_{1}\right)$ and $i \in\{1,2\}$, then $C$ is homogeneous with respect to dense copies of $X$.

7.11. THEOREM. Let $A$ be a Borel subset of the Cantor set. Then for each limit ordinal $\alpha \in\left[\omega, \omega_{1}\right)$ and each $n \in \omega$,

(a) if $A$ is not $\mathscr{P}_{\alpha+2 n}$, then $C$ contains a Cantor set $K$ such that $K \cap A \in \mathscr{X}_{\alpha+2 n+1}^{1}$ and $K \backslash A \in \mathscr{X}_{\alpha+2 n}^{2}$;

(b) if $A$ is not $\mathscr{P}_{\alpha+2 n+1}$, then $C$ contains a Cantor set $K$ such that $K \cap A \in \mathscr{X}_{\alpha+2 n+2}^{1}$ and $K \backslash A \in \mathscr{X}_{\alpha+2 n+1}^{2}$.

We prove the above theorems by induction on $(\beta, i)$, ordered lexicographically, using the following statements as inductive hypotheses (here, $\alpha \in\left[\omega, \omega_{1}\right)$ is a limit ordinal):

If $\beta=\alpha+2 n, i=2$, then

(1) $\left|\mathscr{X}_{\alpha+2 n}^{2}\right|=1$, and if $X \in \mathscr{X}_{\alpha+2 n}^{2}$, then $C$ is homogeneous with respect to dense copies of $X$.

(2) If $A$ is a Borel subset of the Cantor set which is not $\mathscr{P}_{\alpha+2 n}$, then $C$ contains a Cantor set $K$ such that $K \backslash A \in \mathscr{X}_{\alpha+2 n}^{2}$.

If $\beta=\alpha+2 n+1, i=1$, then

(3) Let $X$ be dense and codense in $C$; then $X \in \mathscr{X}_{\alpha+2 n+1}^{1}$ if and only if $C \backslash X \in$ $x_{\alpha+2 n}^{2}$. 
(4) $\left|\mathscr{X}_{\alpha+2 n+1}^{1}\right|=1$, and if $X \in \mathscr{X}_{\alpha+2 n+1}^{1}$, then $C$ is homogeneous with respect to dense copies of $X$.

(5) If $A$ is a Borel subset of the Cantor set which is not $\mathscr{P}_{\alpha+2 n}$, then $C$ contains a Cantor set $K$ such that $K \backslash A \in \mathscr{X}_{\alpha+2 n}^{2}$ and $K \cap A \in \mathscr{X}_{\alpha+2 n+1}^{1}$.

If $\beta=\alpha+2 n+1, i=2$, then

(6) Let $X$ be dense and codense in $C$; then $X \in \mathscr{X}_{\alpha+2 n+1}^{2}$ if and only if $C \backslash X \approx \mathbf{Q}$ $\times Y$ for some $Y \in \mathscr{X}_{\alpha+2 n+1}^{1}$.

(7) $\left|\mathscr{X}_{\alpha+2 n+1}^{2}\right|=1$, and if $X \in \mathscr{X}_{\alpha+2 n+1}^{2}$, then $C$ is homogeneous with respect to dense copies of $X$.

(8) If $A$ is a Borel subset of the Cantor set which is not $\mathscr{P}_{\alpha+2 n+1}$, then $C$ contains a Cantor set $K$ such that $K \backslash A \in \mathscr{X}_{\alpha+2 n+1}^{2}$ and $K \cap A \approx \mathbf{Q} \times Y$ for some $Y \in$ $\mathscr{X}_{\alpha+2 n+1}^{1}$.

If $\beta=\alpha+2 n+2, i=1$, then

(9) Let $X$ be dense and codense in $C$; then $X \in \mathscr{X}_{\alpha+2 n+2}^{1}$ if and only if $C \backslash X \in$ $\mathscr{X}_{\alpha+2 n+1}^{2}$.

(10) $\left|\mathscr{X}_{\alpha+2 n+2}^{1}\right|=1$, and if $X \in \mathscr{X}_{\alpha+2 n+2}^{1}$, then $C$ is homogeneous with respect to dense copies of $X$.

(11) If $A$ is a Borel subset of the Cantor set which is not $\mathscr{P}_{\alpha+2 n+1}$, then $C$ contains a Cantor set $K$ such that $K \backslash A \in \mathscr{X}_{\alpha+2 n+1}^{2}$ and $K \cap A \in \mathscr{X}_{\alpha+2 n+2}^{1}$.

So let $\alpha \in\left[\omega, \omega_{1}\right)$ be a limit ordinal, let $m \in \omega$ and $i \in\{1,2\}$, and suppose that the inductive hypotheses are satisfied for all $(\beta, j)<(\alpha+m, i)$; note that, since $\left|\mathscr{X}_{\beta}^{j}\right|=1$, it follows, as in $\S 6$, that the unique element of $\mathscr{X}_{\beta}^{j}$ is strongly homogeneous.

We now show that the inductive hypotheses are satisfied for $(\alpha+m, i)$; we start with the case $m=0$ (hence $i=2$ ). Of course, we must use the results of $\$ 6$ in case $\alpha=\omega$.

\subsection{LeMMA. $\mathscr{X}_{\alpha}^{2} \neq \varnothing$.}

Proof. Let $\left\{\alpha_{i}: i \in \omega\right\}$ be an increasing sequence of ordinals less than $\alpha$ such that $\sup _{i \in \omega}\left(\alpha_{i}\right)=\alpha$, and let $Y_{i} \in \mathscr{X}_{\alpha_{i}}^{(2)}$ be densely embedded in $C$. Let $\mathbf{Q}=\left\{q_{i}: i \in \omega\right\}$ and put

$$
X=\bigcup_{i \in \omega}\left(\left\{q_{i}\right\} \times Y_{i}\right) \subset \mathbf{Q} \times C .
$$

Then, clearly, $X$ is $\mathscr{P}_{\alpha}$. Also, if $U \times V=\bigcup_{j \in E}\left(\left\{q_{j}\right\} \times V\right) \neq \varnothing$ is clopen in $X$, then $E$ is infinite, and for each $j \in E,\left\{q_{j}\right\} \times V \approx Y_{j}$ is nowhere $\mathscr{P}_{\gamma}$ for all $\gamma<\alpha_{j}$. Since $\left\{q_{j}\right\} \times V$ is closed in $U \times V, U \times V$ is nowhere $\mathscr{P}_{\gamma}$ for each $\gamma<\alpha_{j}$ and each $j \in E$. Hence, $X$ is nowhere $\mathscr{P}_{\gamma}$ for each $\gamma<\alpha$ since $\sup _{j \in E}\left(\alpha_{j}\right)=\alpha$. Therefore, $X \in \mathscr{X}_{\alpha}^{2}$.

\subsection{LEMMA. If $n=0$, then (1) holds.}

Proof. By Lemma 7.12, $\left|\mathscr{X}_{\alpha}^{2}\right| \geqslant 1$; so suppose $X, Y \in \mathscr{X}_{\alpha}^{2}$ are dense subsets of the Cantor set. In analogy to the proof of Lemma 4.6, we construct a homeomorphism $h: C \rightarrow C$ such that $h[X]=Y$. We first consider the case when $\alpha>\omega$.

Since $X$ is $\mathscr{P}_{\alpha}$, we can write $X=\bigcup_{i=0}^{\infty} X_{i}$, where $X_{i}$ is closed in $X$ and $\mathscr{P}_{\alpha_{i}}$ for some $\alpha_{i}<\alpha$; clearly, we may assume that $\alpha_{i} \geqslant \omega$. Note that, since $X$ is nowhere $\mathscr{P}_{\alpha_{i}}$ for 
each $i \in \omega$, each $X_{i}$ is nowhere dense in $X$. Similarly, $Y=\cup_{i=0}^{\infty} Y_{i}$, with $Y_{i}$ closed and nowhere dense in $Y$, and $Y_{i}$ is $\mathscr{P}_{\beta_{i}}$ for some $\beta_{i} \in[\omega, \alpha)$. Let $M$ be as in $\S 4$; we will again construct, for each $s \in M$, collections $\mathscr{U}(s)=\{U(s, n): n \in \mathbf{N}\}, \mathscr{V}(s)=$ $\{V(s, n): n \in \mathbf{N}\}$ of clopen subsets of $C$, closed nowhere dense subsets $D(s), E(s)$ of $C$, and for each $n \in \mathbf{N}$, a homeomorphism $h_{n}: C \rightarrow C$ satisfying the hypotheses of the proof of Lemma 4.6; then, again, $\lim _{n \rightarrow \infty} h_{n}=h: C \approx C$ and $h[X]=Y$. First, let $\gamma_{0}<\alpha$ be an even successor ordinal such that $\alpha_{0}, \beta_{0} \leqslant \gamma_{0}$; then both $X_{0}$ and $Y_{0}$ are $\mathscr{P}_{\gamma_{0}}$. Let $\mathscr{D}$ be a disjoint cover of $C \backslash \bar{X}_{0}$ by clopen subsets of $C$ such that for each $D \in \mathscr{D}, \operatorname{diam}(D)<d\left(D, \bar{X}_{0}\right)$. Since $X$ is a Borel subset of $C$ which is nowhere $\mathscr{P}_{\gamma_{0}+1}, C \backslash X$ is nowhere $\mathscr{P}_{\gamma_{0}}$ by Lemma 7.6. Hence, applying hypothesis (5), each $D \in \mathscr{D}$ contains a Cantor set $K(D)$ such that $K(D) \cap X=E(D) \in \mathscr{X}_{\gamma_{0}}^{2}$. Then

$$
A_{0}=X_{0} \cup \bigcup_{D \in \mathscr{D}} E(D)
$$

is closed in $X$ and strongly $\sigma-\mathscr{P}_{\gamma_{0}}$, hence $\mathscr{P}_{\gamma_{0}}$ by Corollary 7.5. As in the proof of Lemma 6.16, it can be shown that, for each open subset $U$ of $A_{0}, U \cap E(D) \neq \varnothing$ for some $D \in \mathscr{D}$; hence $A_{0}$ is nowhere $\mathscr{P}_{\gamma_{0}-1}$, since $E(D)$ is nowhere $\mathscr{P}_{\gamma_{0}-1}$. Also, $U \cap E(D)$ contains a subset $B$ which is closed in $E(D)$, hence in $A_{0}$, such that $B \in \mathscr{X}_{\gamma_{0}-1}^{2}$; thus $A_{0} \in \mathscr{X}_{\gamma_{0}}^{2}$. Similarly, we can find a closed subset $B_{0}$ of $Y$ such that $Y_{0} \subset B_{0}$ and $B_{0} \in \mathscr{X}_{\gamma_{0}}^{2}$; note that $A_{0}$ is nowhere dense in $X$, and $B_{0}$ is nowhere dense in $Y$. Now put $D(\varnothing)=\bar{A}_{0}, E(\varnothing)=\bar{B}_{0}$; by hypothesis (1) there exists a homeomorphism $h^{0}: D(\varnothing) \rightarrow E(\varnothing)$ such that $h^{0}\left[A_{0}\right]=B_{0}$. We construct $\mathscr{U}(\varnothing), \mathscr{V}(\varnothing)$, and $h_{1}$ as in the proof of Lemma 4.6.

If $D(s), E(s), \mathscr{U}(s), \mathscr{V}(s)$, and $h_{m}$ have been constructed for $|s|<n(\geqslant 1)$ and $m \leqslant n$, then fix $s \in M$ with $|s|=n-1$. Let

$$
k_{i}=\min \left\{j: U(s, i) \cap X_{j} \neq \varnothing\right\}, \quad l_{i}=\min \left\{j: V(s, i) \cap Y_{j} \neq \varnothing\right\}
$$

and find an even successor $\gamma(s, i)<\alpha$ such that $\alpha_{k_{i}}, \beta_{l_{i}} \leqslant \gamma(s, i)$; then both $U(s, i) \cap X_{k_{i}}$ and $V(s, i) \cap Y_{l_{i}}$ are $\mathscr{P}_{\gamma(s, i)}$. As above, we obtain closed subsets $A(s, i)$

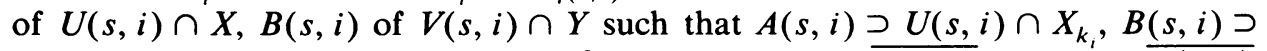
$V(s, i) \cap Y_{l_{i}}$, and $A(s, i), B(s, i) \in \mathscr{X}_{\gamma(s, i)}^{2}$. Put $D(s, i)=\overline{A(s, i)}, E(s, i)=\overline{B(s, i)}$, and let $h^{0}(s, i): D(s, i) \rightarrow E(s, i)$ be a homeomorphism such that $h^{0}(s, i)[A(s, i)]$ $=B(s, i)$, applying hypothesis (1); now finish the proof as in Lemma 4.6.

In case $\alpha=\omega$, the proof is similar; the sets $A_{0}, B_{0}, A(s, i)$, and $B(s, i)$ should be chosen in some $\mathscr{X}_{4 k+2}^{i}$ or $\mathscr{X}_{4 k+3}^{i}$ because we want to apply Theorem 6.14.

7.14. LEMMA. If $n=0$, then (2) holds.

Proof. For the sake of simplicity, assume that $\alpha>\omega$ and let $A$ be a Borel subset of the Cantor set which is not $\mathscr{P}_{\alpha}$. Let $\left\{\alpha_{i}: i \in \omega\right\}$ be an increasing sequence of even ordinals in $[\omega, \alpha)$ such that $\sup _{i \in \omega}\left(\alpha_{i}\right)=\alpha$, and let $Z_{i}$ be the unique element of $\mathscr{X}_{\alpha_{i}+1}^{1}$. Put $\mathscr{P}=\mathscr{P}_{\alpha}$; then $\mathscr{P}$ is closed-hereditary by Lemma 7.1, and $\mathscr{P}$ is strongly $\sigma$-additive by Corollary 7.3.

Let $B$ be a Borel subset of the Cantor set which is not $\mathscr{P}$. Then for each $i \in \omega, B$ is not $\mathscr{P}_{\alpha_{i}}$, hence by hypothesis (5), $C$ contains a Cantor set $K_{i}$ such that $K_{i} \backslash B \in \mathscr{X}_{\alpha_{i}+1}^{1}$ and $K_{i} \cap B \in \mathscr{X}_{\alpha_{i}}^{2}$. Thus, $K_{i} \backslash B \approx Z_{i}$, and $K_{i} \cap B$ is $\mathscr{P}_{\alpha_{i}}$, hence $\mathscr{P}$. So by Lemma 
5.3, $C$ contains Cantor sets $B_{i}$, for $i \in \omega$, such that $K=\left(\cup_{i=0}^{\infty} B_{i}\right)^{-} \approx C$ and, for each $i \in \omega$,

(i) $B_{i}$ is nowhere dense in $K$;

(ii) $K \backslash A=\bigcup_{i=0}^{\infty}\left(B_{i} \backslash A\right)$;

(iii) $B_{0} \backslash A \approx Z_{0},\left(B_{i+1} \backslash B_{i}\right) \backslash A \approx Z_{i+1}$.

Since $\left(B_{i+1} \backslash B_{i}\right) \backslash A$ is open in $B_{i+1} \backslash A$, we can write

$$
\left(B_{i+1} \backslash B_{i}\right) \backslash A=\bigcup_{j=0}^{\infty} K_{j}^{i},
$$

where $K_{j}^{i}$ is clopen in $B_{i+1} \backslash A$; then $K_{j}^{i} \approx Z_{i+1}$ is $\mathscr{P}_{\alpha_{i+1}+1}$, so

$$
K \backslash A=\left(B_{0} \backslash A\right) \cup \bigcup_{i, j=0}^{\infty} K_{j}^{i}
$$

is $\mathscr{P}_{\alpha}$. By (i) each open subset of $K \backslash A$ intersects some $K_{j_{i}}^{i}$ for infinitely many $i$; hence, since $K_{j_{i}}^{i}$ is nowhere $\mathscr{P}_{\beta}$ for each $\beta<\alpha_{i}, K \backslash A$ is nowhere $\mathscr{P}_{\beta}$ for each $\beta<\alpha$. Thus $K \backslash A \in \mathscr{X}_{\alpha}^{2}$.

Lemmas 7.13 and 7.14 show that the inductive hypotheses are satisfied for $(\alpha+m, i)$ if $m=0$. We now turn to the case where $m=2 n+1$ for some $n \in \omega$ and $i=1$.

\subsection{LEMMA. (3) holds.}

Proof. First, let $X \in \mathscr{X}_{\alpha+2 n+1}^{1}$; then no closed subset of $X$ belongs to $\mathscr{X}_{\alpha+2 n}^{2}$, and hence $C \backslash X$ is $\mathscr{P}_{\alpha+2 n}$ by hypothesis (2). Let $U$ be a nonempty clopen subset of $C$.

Case 1: $n=0$. Suppose $U \backslash X$ is $\mathscr{P}_{\beta}$ for some $\beta<\alpha$. By Lemma 6.4 and 7.6, $U \cap X$ is $\mathscr{P}_{\gamma}$ for some $\gamma<\alpha$, a contradiction; hence $C \backslash X \in \mathscr{X}_{\alpha}^{2}$.

Case 2: $n>0$. Suppose $U \backslash X$ is $\mathscr{P}_{\alpha+2 n-1}$. By Lemma 7.6, $U \cap X$ is $\mathscr{P}_{\alpha+2 n}$, a contradiction; hence $C \backslash X$ is nowhere $\mathscr{P}_{\alpha+2 n-1}$. Also, since $U \cap X$ is not $\mathscr{P}_{\alpha+2 n-1}$, we obtain from hypothesis (8) a Cantor set $K$ in $U$ such that $K \backslash X \in \mathscr{X}_{\alpha+2 n-1}^{2}$; so $C \backslash X \in \mathscr{X}_{\alpha+2 n}^{2}$.

Conversely, suppose $C \backslash X \in \mathscr{X}_{\alpha+2 n}^{2}$; then by Lemma 7.6, $X$ is $\mathscr{P}_{\alpha+2 n+1}$. Let $U$ be a nonempty clopen subset of $C$.

Case 1: $n=0$. As in Case 1 above, $X$ is nowhere $\mathscr{P}_{\beta}$ for each $\beta<\alpha$. Suppose $U \cap X$ is $\mathscr{P}_{\alpha}$; then $U \cap X \in \mathscr{X}_{\alpha}$, so $U \cap X$ is not Baire by Lemma 7.8. Hence, $U \backslash X \in \mathscr{X}_{\alpha}^{2}$ is Baire, contradicting Lemma 7.8; so $X$ is nowhere $\mathscr{P}_{\alpha}$. Finally, suppose $X$ contains a closed subset $B$ belonging to $\mathscr{X}_{\alpha}^{2}$. By the same argument as above, $\bar{B} \backslash B$ is nowhere $\mathscr{P}_{\alpha}$; but $\bar{B} \backslash B$ is a closed subset of $C \backslash X$, and this contradicts Lemma 7.1.

Case 2: $n>0$. We must show that $U \cap X$ is not $\mathscr{P}_{\alpha+2 n}$. Since $U \backslash X$ is nowhere $\mathscr{P}_{\alpha+2 n-1}, U \cap X$ is nowhere $\mathscr{P}_{\alpha+2 n-2}$ by Lemma 7.6. Assume that $U \cap X$ is $\mathscr{P}_{\alpha+2 n-1}$; since $U \backslash X$ is nowhere $\mathscr{P}_{\alpha+2 n-2}$ (Corollary 7.3), it follows from hypothesis (5) that every clopen subset of $U \cap X$ contains a closed subset belonging to $\mathscr{X}_{\alpha+2 n-2}^{2}$, and hence $U \cap X \in \mathscr{X}_{\alpha+2 n-1}^{2}$. But then by hypothesis (9), $U \backslash X \in \mathscr{X}_{\alpha+2 n}^{1}$, so $U \backslash X$ is a nonempty clopen subset of $C \backslash X$ which contains no closed subset belonging to $\mathscr{X}_{\alpha+2 n-1}^{2}$, contradicting $C \backslash X \in \mathscr{X}_{\alpha+2 n}^{2}$; so $U \cap X$ is not $\mathscr{P}_{\alpha+2 n-1}$. But then $U \cap X$ is not $\mathscr{P}_{\alpha+2 n}$ either, since by Lemma 7.4, this would imply that $U \cap X$ is 
not Baire, and hence that $U \backslash X \in \mathscr{X}_{\alpha+2 n}^{2}$ is Baire, contradicting Lemma 7.8. It remains to show that $X$ contains no closed subsets belonging to $\mathscr{X}_{\alpha+2 n}^{2}$. So suppose to the contrary that $X$ contains such a subset $B$. By the above argument $\bar{B} \backslash B$ is nowhere $\mathscr{P}_{\alpha+2 n}$, but $\bar{B} \backslash B$ is a closed subset of $C \backslash X \in \mathscr{X}_{\alpha+2 n}^{2}$, a contradiction with Lemma 7.1.

\subsection{LEMMA. (4) and (5) hold.}

Proof. By hypothesis (1), $\mathscr{X}_{\alpha+2 n}^{2} \neq \varnothing$, say $X \in \mathscr{X}_{\alpha+2 n}^{2}$. Embed $X$ densely in $C$; then by Lemma 7.15, $C \backslash X \in \mathscr{X}_{\alpha+2 n+1}^{1}$, so $\left|\mathscr{X}_{\alpha+2 n+1}^{1}\right| \geqslant 1$. And if $X, Y \in \mathscr{X}_{\alpha+2 n+1}^{1}$ are dense subsets of $C$, then by Lemma 7.15, $C \backslash X, C \backslash Y \in \mathscr{X}_{\alpha+2 n}^{2}$; hence, by (1), there exists a homeomorphism $h: C \rightarrow C$ such that $h[C \backslash X]=C \backslash Y$, and thus also $h[X]=Y$. This proves (4). To prove (5), let $K^{\prime}$ be the Cantor set obtained from (2) and put $K=\overline{K^{\prime} \backslash A}$; then $K \cap A \in \mathscr{X}_{\alpha+2 n+1}^{1}$ by Lemma 7.15.

The next case is $m=2 n+1$ for some $n \in \omega$ and $i=2$; we must show that (6)-(8) are valid.

\subsection{LEMMA. (6) holds.}

Proof. First, let $X \in \mathscr{X}_{\alpha+2 n+1}^{2}$. Then $X$ is $\mathscr{P}_{\alpha+2 n+1}$, so we can write $X=A \cup B$, where $A$ is $\mathscr{P}_{\alpha+2 n}$ and $B$ is complete. Put $C \backslash B=\bigcup_{i=1}^{\infty} K_{i}$, where $K_{i}$ is compact and let $L_{i}=K_{i} \backslash X$; then $L_{i}$ is closed in $C \backslash X$ and $C \backslash X=\bigcup_{i=1}^{\infty} L_{i}$. Since $K_{i} \cap X$ is a closed subset of $A, K_{i} \cap X$ is $\mathscr{P}_{\alpha+2 n}$, and hence $L_{i}$ is $\mathscr{P}_{\alpha+2 n+1}$, by Lemma 7.6. We show that $L_{i}$ is nowhere dense in $C \backslash X$ and $L_{i}$ does not contain any closed subsets belonging to $\mathscr{X}_{\alpha+2 n}^{2}$. We then construct closed nowhere dense subsets $A_{i}$ of $C \backslash X$ such that $L_{i} \subset A_{i} \in \mathscr{X}_{\alpha+2 n+1}^{1}$; since $\left|\mathscr{X}_{\alpha+2 n+1}^{1}\right|=1$ by (4), it then follows from Theorem 4.3 that $C \backslash X \approx \mathbf{Q} \times Y$ for $Y \in \mathscr{X}_{\alpha+2 n+1}^{1}$.

Now let $U \neq \varnothing$ be clopen in $C$ and suppose $U \backslash X$ is $\mathscr{P}_{\alpha+2 n}$.

Case 1: $n=0$. Since $U \cap X$ is nowhere $\mathscr{P}_{\alpha}, U \backslash X$ is nowhere $\mathscr{P}_{\beta}$ for each $\beta<\alpha$, so $U \backslash X \in \mathscr{X}_{\alpha}^{2}$.

Case 2: $n>0$. Since $U \cap X$ is nowhere $\mathscr{P}_{\alpha+2 n}, U \backslash X$ is nowhere $\mathscr{P}_{\alpha+2 n-1}$ by Lemma 7.6. Let $V$ be a nonempty clopen subset of $U$. Since $V \cap X$ is not $\mathscr{P}_{\alpha+2 n-1}$, we can apply (8) to obtain a closed subset of $V \backslash X$ which belongs to $\mathscr{X}_{\alpha+2 n-1}^{2}$; hence $U \backslash X \in \mathscr{X}_{\alpha+2 n}^{2}$.

So, in both cases, if $U \backslash X$ is $\mathscr{P}_{\alpha+2 n}$, then $U \backslash X \in \mathscr{X}_{\alpha+2 n}^{2}$. But then by (3), $U \cap X \in \mathscr{X}_{\alpha+2 n+1}^{1}$ is a nonempty clopen subset of $X$ containing no closed subset belonging to $\mathscr{X}_{\alpha+2 n}^{2}$, contradicting $X \in \mathscr{X}_{\alpha+2 n+1}^{2}$; hence $C \backslash X$ is nowhere $\mathscr{P}_{\alpha+2 n}$. From this it follows that if $U \backslash X$ were $\mathscr{P}_{\alpha+2 n+1}$, say $U \backslash X=G \cup H$, where $G$ is $\mathscr{P}_{\alpha+2 n}$ and $H$ is complete, then $H$ would be a dense complete subset of $U \backslash X$; this contradicts the Baire category theorem for $U \approx C$, since $U \cap X$ contains a dense complete subset as well (Lemma 7.8). Thus, $C \backslash X$ is nowhere $\mathscr{P}_{\alpha+2 n+1}$, and, in particular, each $L_{i}$ is nowhere dense in $C \backslash X$.

If $B$ is a closed subset of $L_{i}$ belonging to $\mathscr{X}_{\alpha+2 n}^{2}$, then $\bar{B} \backslash B \in \mathscr{X}_{\alpha+2 n+1}^{1}$ by (3); hence, $\bar{B} \backslash B$ is not $\mathscr{P}_{\alpha+2 n}$; but, on the other hand, $\bar{B} \backslash B=\bar{B} \cap X \subset K_{i} \cap X \subset A$ is a closed subset of $A$, and $A$ is $\mathscr{P}_{\alpha+2 n}$; hence, $\bar{B} \backslash B$ is $\mathscr{P}_{\alpha+2 n}$ by Lemma 7.1. Thus, no closed subset of $L_{i}$ belongs to $\mathscr{X}_{\alpha+2 n}^{2}$. Fix $i \in \mathbf{N}$ and let $\mathscr{D}$ be a disjoint cover of 
$(C \backslash X) \backslash L_{i}$ by clopen subsets of $C \backslash X$ such that for each $D \in \mathscr{D}, \operatorname{diam}(D)<$ $d\left(D, L_{i}\right)$. Since $C \backslash X$ is nowhere $\mathscr{P}_{\alpha+2 n}$, it follows from (5) that each $D \in \mathscr{D}$ contains a closed subset $E(D) \in \mathscr{X}_{\alpha+2 n+1}^{1}$. Then

$$
A_{i}=L_{i} \cup \bigcup_{D \in \mathscr{D}} E(D)
$$

is closed in $C \backslash X$ and $\mathscr{P}_{\alpha+2 n+1}$, hence nowhere dense. Clearly, $A_{i}$ contains no closed subsets belonging to $\mathscr{X}_{\alpha+2 n}^{2}$, and since each open subset of $A_{i}$ intersects some $E(D)$, $A_{i}$ is nowhere $\mathscr{P}_{\alpha+2 n}$; hence $A_{i} \in \mathscr{X}_{\alpha+2 n+1}^{1}$. This proves the first part of the lemma.

Conversely, assume that $C \backslash X \approx \mathbf{Q} \times Y$ for $Y \in \mathscr{X}_{\alpha+2 n+1}^{1}$, say $C \backslash X=\bigcup_{i=1}^{\infty} A_{i}$, where each $A_{i} \in \mathscr{X}_{\alpha+2 n+1}^{1}$ is a closed nowhere dense subset of $C \backslash X$. Then

$$
X=\bigcup_{i=1}^{\infty}\left(\overline{A_{i}} \backslash A_{i}\right) \cup C \backslash \bigcup_{i=1}^{\infty} \overline{A_{i}} .
$$

By (3), $\overline{A_{i}} \backslash A_{i} \in \mathscr{X}_{\alpha+2 n}^{2}$; hence $X$ is strongly $\sigma-\mathscr{P}_{\alpha+2 n} \cup$ complete is $\mathscr{P}_{\alpha+2 n+1}$ by Corollary 7.5; and since $\bigcup_{i=1}^{\infty}\left(\overline{A_{i}} \backslash A_{i}\right)$ is dense in $X$, every nonempty clopen subset of $X$ contains a closed subset belonging to $\mathscr{X}_{\alpha+2 n}^{2}$. It remains to be shown that $X$ is nowhere $\mathscr{P}_{\alpha+2 n}$; so let $U$ be a nonempty clopen subset of $C$.

Case 1: $n=0$. Suppose $U \cap X$ is $\mathscr{P}_{\beta}$ for some $\beta<\alpha$; then $U \backslash X$ is $\mathscr{P}_{\gamma}$ for some $\gamma<\alpha$; hence for each $i, A_{i} \cap(U \backslash X)$ is $\mathscr{P}_{\gamma}$. Since $A_{i} \cap(U \backslash X) \neq \varnothing$ for some $i$, this contradicts the fact that $A_{i}$ is nowhere $\mathscr{P}_{\gamma}$ for each $\gamma<\alpha$; hence, $U \cap X$ is nowhere $\mathscr{P}_{\beta}$ for each $\beta<\alpha$. So if $U \cap X$ were $\mathscr{P}_{\alpha}$, it would not be Baire; but $U \backslash X$ is not Baire either, a contradiction.

Case 2: $n>0$. Suppose $U \cap X$ is $\mathscr{P}_{\alpha+2 n-1}$; then $U \backslash X$ is $\mathscr{P}_{\alpha+2 n}$ by Lemma 7.6, and hence $A_{i} \cap(U \backslash X)$ is $\mathscr{P}_{\alpha+2 n}$ for each $i$. Since $A_{i} \cap(U \backslash X) \neq \varnothing$ for some $i$, this contradicts the fact that $A_{i}$ is nowhere $\mathscr{P}_{\alpha+2 n}$; hence, $U \cap X$ is nowhere $\mathscr{P}_{\alpha+2 n-1}$. Again, if $U \cap X$ were $\mathscr{P}_{\alpha+2 n}$, hence strongly $\sigma-\mathscr{P}_{\alpha+2 n-1}$ by Lemma 7.4, then $U \cap X$ would not be Baire, and, as in Case 1, we obtain a contradiction.

\subsection{LEMMA. (7) holds.}

Proof. By (4), $\mathscr{X}_{\alpha+2 n+1}^{1} \neq \varnothing$, say $Y \in \mathscr{X}_{\alpha+2 n+1}^{1}$. Let $X$ be a dense copy of $\mathbf{Q} \times Y$ in the Cantor set; then by Lemma 7.17, $C \backslash X \in \mathscr{X}_{\alpha+2 n+1}^{2}$, and hence $\mathscr{X}_{\alpha+2 n+1}^{2} \neq \varnothing$. Now assume that $X_{1}, X_{2} \in \mathscr{X}_{\alpha+2 n+1}^{2}$ are dense subsets of the Cantor set; by Lemma 7.17, and since $\left|\mathscr{X}_{\alpha+2 n+1}^{1}\right|=1$, we have $C \backslash X_{1} \approx C \backslash X_{2} \approx \mathbf{Q} \times Y$ for some $Y \in$ $\mathscr{X}_{\alpha+2 n+1}^{1}$. By Theorem 4.7 and (4) there exists a homeomorphism $h: C \rightarrow C$ such that $h\left[C \backslash X_{1}\right]=C \backslash X_{2}$; hence $h\left[X_{1}\right]=X_{2}$.

\subsection{LEMMA. (8) holds.}

Proof. Let $\mathscr{P}=\mathscr{P}_{\alpha+2 n}$, and let $Z$ be the unique element of $\mathscr{X}_{\alpha+2 n+1}^{1}$. We show that the hypotheses of Lemma 5.2 are satisfied. By Lemma 7.1, $\mathscr{P}$ is closed-hereditary; by Corollary 7.5, $\mathscr{P}$ is strongly $\sigma$-additive. Let $B$ be a Borel subset of the Cantor set which is not $\mathscr{P}$; by (5), $C$ contains a Cantor set $K$ such that $K \backslash B \in X_{\alpha+2 n}^{2}$ and $K \cap B \in \mathscr{X}_{\alpha+2 n+1}^{1}$. Then $K \cap B \approx Z$ and $K \backslash B=\bigcup_{i=1}^{\infty} X_{i}$, where $X_{i}$ is closed in $K \backslash B$ and $\mathscr{P}_{\beta_{i}}$ for some $\beta_{i}<\alpha$ (if $n=0$ ), respectively, $\mathscr{P}_{\alpha+2 n-1}$ (if $n>0$, applying Lemma 7.4). Put $G=\cup_{i=1}^{\infty} \bar{X}_{i}$; then $G$ is $\sigma$-compact, and $G \cap B=\cup_{i=1}^{\infty}\left(\bar{X}_{i} \backslash X_{i}\right)$ is 
$\mathscr{P}_{\alpha}$ (if $n=0$ ), respectively, strongly $\sigma-\mathscr{P}_{\alpha+2 n}$ and, hence, $\mathscr{P}_{\alpha+2 n}$ (if $n>0$, applying Lemma 7.6 and Corollary 7.5). So by Lemma 5.2, if $A$ is a Borel subset of $C$ which is not $\mathscr{P}_{\alpha+2 n} \cup$ complete, then $C$ contains a Cantor set $K^{\prime}$ such that $K^{\prime} \cap A \approx \mathbf{Q} \times Z$. Put $K=\overline{K^{\prime} \cap A}$; then $K \approx C, K \cap A \approx \mathbf{Q} \times Z$, and $K \backslash A \in \mathscr{X}_{\alpha+2 n+1}^{1}$ by Lemma 7.17 .

We now consider the case $m=2 n+2$ for some $n \in \omega$ and $i=1$ (recall that if $m=0$, we only have the case $(\alpha+m, 2))$; we must show that hypotheses (9)-(11) are true for this $n$.

7.20. Lemma. Let $Y$ be the unique element of $\mathscr{X}_{\alpha+2 n+1}^{1}$; then $\mathbf{Q} \times Y$ is the unique element of $\mathscr{X}_{\alpha+2 n+2}^{1}$ up to homeomorphism.

Proof. We first show that $\mathbf{Q} \times Y \in \mathscr{X}_{\alpha+2 n+2}^{1}$. Clearly, $\mathbf{Q} \times Y=\bigcup_{q \in \mathbf{Q}}(\{q\} \times Y)$ is strongly $\sigma-\mathscr{P}_{\alpha+2 n+1}$ is $\mathscr{P}_{\alpha+2 n+2}$ by Lemma 7.4. Note that since every clopen subset of $\mathbf{Q} \times Y$ contains a closed copy of $Y$, and $Y$ is not $\mathscr{P}_{\alpha+2 n}, \mathbf{Q} \times Y$ is nowhere $\mathscr{P}_{\alpha+2 n}$ by Lemma 7.1. Suppose $U \times V$ is a nonempty basic clopen subset of $\mathbf{Q} \times Y$ which is $\mathscr{P}_{\alpha+2 n+1}$; then we can write $U \times V=A \cup B$, where $A$ is $\mathscr{P}_{\alpha+2 n}$ and $B$ is complete. Since $U \times V$ is nowhere $\mathscr{P}_{\alpha+2 n}$ by the preceding remark, $B$ is dense in $U \times V$, contradicting the fact that $U \times V$ is not Baire; so $\mathbf{Q} \times Y$ is nowhere $\mathscr{P}_{\alpha+2 n+1}$. It remains to show that $\mathbf{Q} \times Y$ contains no closed subset belonging to $\mathscr{X}_{\alpha+2 n+1}^{2}$; so embed $\mathbf{Q} \times Y$ as a dense subset of the Cantor set, and suppose to the contrary that $B \in \mathscr{X}_{\alpha+2 n+1}^{2}$ is closed in $\mathbf{Q} \times Y$. Then by (6), $\bar{B} \backslash B \approx \mathbf{Q} \times Y$, so $\bar{B} \backslash B$ is not $\mathscr{P}_{\alpha+2 n+1}$ by the above argument. On the other hand, $\bar{B} \backslash B$ is a closed subset of $C \backslash(\mathbf{Q} \times Y)$, which belongs to $\mathscr{X}_{\alpha+2 n+1}^{2}$ by another application of (6); so $\bar{B} \backslash B$ is $\mathscr{P}_{\alpha+2 n+1}$ by Lemma 7.1, and we have the required contradiction.

To show that $\mathbf{Q} \times Y$ is the only element of $\mathscr{X}_{\alpha+2 n+2}^{1}$, it suffices to show that, if $X \in \mathscr{X}_{\alpha+2 n+2}^{1}$ is a dense subset of $C$, then $C \backslash X \in \mathscr{X}_{\alpha+2 n+1}^{2}$; hypothesis (6) then yields that $X \approx \mathbf{Q} \times Y$. First note that, since $X$ contains no closed subset belonging to $\mathscr{X}_{\alpha+2 n+1}^{2}$, it follows from (8) that $C \backslash X$ is $\mathscr{P}_{\alpha+2 n+1}$. Also, since $X$ is nowhere $\mathscr{P}_{\alpha+2 n+1}, C \backslash X$ is nowhere $\mathscr{P}_{\alpha+2 n}$ by Lemma 7.6. And if $U$ is clopen in $C$, then, since $U \cap X$ is not $\mathscr{P}_{\alpha+2 n}$, we can apply (2) and obtain a closed subset of $U \backslash X$ belonging to $\mathscr{X}_{\alpha+2 n}^{2}$.

7.21. Lemma. (9), (10), and (11) hold.

Proof. (9) follows from (6) and Lemma 7.20. If $X_{1}, X_{2} \in \mathscr{X}_{\alpha+2 n+2}^{1}$ are dense subsets of the Cantor set, then $C \backslash X_{1}, C \backslash X_{2} \in \mathscr{X}_{\alpha+2 n+1}^{2}$, so by (7) there exists a homeomorphism $h: C \rightarrow C$ such that $h\left[C \backslash X_{1}\right]=C \backslash X_{2}$, and hence $h\left[X_{1}\right]=X_{2}$. (11) follows from (8) and Lemma 7.20.

Finally, we have to show that the hypotheses are satified if $m=2 n+2$ for some $n \in \omega$ and $i=2$; i.e., we must show that (1) and (2) hold if $n>0$.

7.22. LemMA. Let $n \in \mathbf{N}$, and let $Y$ be the unique element of $\mathscr{X}_{\alpha+2 n-1}^{2}$; then $\mathbf{Q} \times Y$ is the unique element of $\mathscr{X}_{\alpha+2 n}^{2}$ up to homeomorphism.

Proof. As in the proof of Lemma 7.20, it can be shown that $\mathbf{Q} \times Y$ is $\mathscr{P}_{\alpha+2 n}$ and nowhere $\mathscr{P}_{\alpha+2 n-1}$; and since $Y$ is strongly homogeneous, every nonempty clopen 
subset of $\mathbf{Q} \times Y$ contains a closed copy of $Y$. Hence, $\mathbf{Q} \times Y \in \mathscr{X}_{\alpha+2 n}^{2}$. Now suppose that $X \in \mathscr{X}_{\alpha+2 n}^{2}$. Then $X$ is $\mathscr{P}_{\alpha+2 n}$, so by Lemma 7.4 we can write $X=\bigcup_{i=1}^{\infty} X_{i}$, where each $X_{i}$ is closed in $X$ and $\mathscr{P}_{\alpha+2 n-1}$. Since $X$ is nowhere $\mathscr{P}_{\alpha+2 n-1}$, each $X_{i}$ is nowhere dense in $X$. Fix $i \in \mathbf{N}$, and let $\mathscr{D}$ be a disjoint cover of $X \backslash X_{i}$ by clopen subsets of $X$ such that $\operatorname{diam}(D)<d\left(D, X_{i}\right)$ for each $D \in \mathscr{D}$. Then each $D \in \mathscr{D}$ contains a closed copy $E(D)$ of $Y$, and, as before, it is easily shown that

$$
A_{i}=X_{i} \cup \bigcup_{D \in \mathscr{D}} E(D)
$$

is a closed nowhere dense subset of $X$ such that $A_{i} \approx Y$. Hence, $X=\cup_{i=1}^{\infty} A_{i} \approx \mathbf{Q} \times Y$ by Theorem 4.3 .

7.23. LEMMA. If $n>0$, then (1) holds.

Proof. By Lemma 7.22, $\left|\mathscr{X}_{\alpha+2 n}^{2}\right|=1$. So suppose that $X_{1}, X_{2} \in \mathscr{X}_{\alpha+2 n}^{2}$ are dense in $C$. If $Y$ is the unique element of $\mathscr{X}_{\alpha+2 n-1}^{2}$ (hypothesis (7)), then $X_{1} \approx X_{2} \approx \mathbf{Q} \times Y$ by Lemma 7.22, so by (7) we can apply Theorem 4.7 to obtain a homeomorphism $h$ : $C \rightarrow C$ such that $h\left[X_{1}\right]=X_{2}$.

7.24. LEMMA. If $n>0$, then (2) holds.

Proof. Let $\mathscr{P}=\mathscr{P}_{\alpha+2 n}$, and let $Z$ be the unique element of $\mathscr{X}_{\alpha+2 n-1}^{2}$. By Lemma $7.1, \mathscr{P}$ is closed-hereditary; by Corollary $7.5, \mathscr{P}$ is strongly $\sigma$-additive. Let $B$ be a Borel subset of the Cantor set which is not $\mathscr{P}$. Then by Lemma 7.6, $C \backslash B$ is not $\mathscr{P}_{\alpha+2 n-1}$, and hence, by (11), $C$ contains a Cantor set $K$ such that $K \backslash B \in \mathscr{X}_{\alpha+2 n-1}^{2}$ and $K \cap B \in \mathscr{X}_{\alpha+2 n}^{1}$; so $K \backslash B \approx Z$ and $K \cap B$ is $\mathscr{P}$. Thus, by Lemma 5.3, we can find Cantor sets $B_{i}$ in $C$, for each $i \in \omega$, such that $K=\left(\cup_{i=0}^{\infty} B_{i}\right)^{-} \approx C$ and for each $i \in \omega$,

(i) $B_{i}$ is nowhere dense in $K$;

(ii) $K \backslash A=\bigcup_{i=0}^{\infty}\left(B_{i} \backslash A\right)$;

(iii) $B_{0} \backslash A \approx Z \approx\left(B_{i+1} \backslash B_{i}\right) \backslash A$.

As in the proof of Lemma 6.23, we deduce from Theorem 4.3 that $K \backslash A \approx \mathbf{Q} \times Z$, and hence by Lemma 7.22, $K \backslash A \in \mathscr{X}_{\alpha+2 n}^{2}$.

This completes the proofs of Theorems 7.9-7.11. We further note that Remark 6.24 also applies to Theorem 7.11.

As in $\$ 6$, we also finish this section with the statement that all spaces defined above are topologically different.

7.25. TheOREM. Let $\beta, \gamma \in\left[\omega, \omega_{1}\right)$ and $i, j \in\{1,2\}$ be such that $i=2$ if $\beta$ is a limit ordinal and $j=2$ if $\gamma$ is a limit ordinal. If $(\beta, i) \neq(\gamma, j)$, then $\mathscr{X}_{\beta}^{i} \cap \mathscr{X}_{\gamma}^{j}=\varnothing$.

Proof. If $\beta \neq \gamma$, say $\beta<\gamma$, then $X \in \mathscr{X}_{\gamma}^{j}$ is nowhere $\mathscr{P}_{\beta}$ by Corollary 7.3 , so $X \notin \mathscr{X}_{\beta}^{i}$. If $\beta=\gamma$, then $i \neq j$, say $i=1, j=2$; hence, $X \in \mathscr{X}_{\gamma}^{j}$ contains a closed subset belonging to $\mathscr{X}_{\gamma-1}^{2}$, whereas $X \in \mathscr{X}_{\beta}^{i}$ does not.

8. The main theorem. In this section we show that the spaces, defined and characterized in $\$ \S 6$ and 7 , are the only zero-dimensional, homogeneous, non- $\sigma$ compact Borel sets of ambiguous class 2 in the Cantor set. 
8.1. Lemma. Let $X$ be homogeneous and zero-dimensional.

(a) If $\gamma \in\left[-1, \omega_{1}\right), i \in\{1,2\}$, and $X$ is not $\mathscr{P}_{\gamma}^{(i)}$, then $X$ is nowhere $\mathscr{P}_{\gamma}^{(i)}$.

(b) If $Y$ is a strongly homogeneous closed subset of $X$, then every nonempty clopen subset of $X$ contains a closed copy of $Y$.

Proof. (a) Suppose $U$ is a nonempty clopen subset of $X$ which is $\mathscr{P}_{\gamma}^{(i)}$. Let $x \in U$, and for each $y \in X$, let $h_{y}: X \rightarrow X$ be a homeomorphism such that $h_{y}(x)=y$. If $\left\{U_{i}: i \in \mathbf{N}\right\}$ is a countable subcover of $\left\{h_{y}[U]: y \in X\right\}$, then for each $n \in \mathbf{N}$, the clopen set $V_{n}=U_{n} \backslash U_{i<n} U_{i}$ is $\mathscr{P}_{\gamma}^{(i)}$ by Lemmas 6.1 or 7.1. Hence, $X=\bigoplus_{n \in \mathrm{N}} V_{n}$ is also $\mathscr{P}_{\gamma}^{(i)}$.

(b) Let $U$ be a nonempty clopen subset of $X, x \in U$ and $y \in Y$. If $h: X \rightarrow X$ is a homeomorphism such that $h(y)=x$, then $h[Y] \cap U$ is a nonempty clopen subset of $h[Y] \approx Y$, so $h[Y] \cap U \approx Y$, since $Y$ is strongly homogeneous. Since $h[Y]$ is closed in $X, h[Y] \cap U$ is closed in $U$.

8.2. THEOREM. Let $X$ be a non-б-compact, homogeneous, zero-dimensional absolute Borel set of ambiguous class 2. Then for some ordinal $\alpha<\omega_{1}$ and some $i \in\{1,2\}$, $X \in \mathscr{X}_{\alpha}^{(i)}$.

Proof. By Theorems 3.2 and 3.3, $X \in \mathscr{K}_{\gamma}$ for some even $\gamma<\omega_{1}$; let $\alpha<\omega_{1}$ be minimal such that $X \in \mathscr{K}_{\alpha}$.

Case 1: $\alpha<\omega$. Say $\alpha=2 n$ for some $n \in \omega$. Then if $n=0, X$ is complete and nowhere $\sigma$-compact by Lemma 8.1(a), so $X \in \mathscr{X}_{0}$. If $n>0$, then $X$ is $\Sigma_{n}$ is $\mathscr{P}_{4(n-1)+1}$. Let $<$ be the ordering of the properties $\mathscr{P}_{m}^{(j)}$ defined in Theorem 6.25, and let $\mathscr{P}_{k}^{(i)}$ be minimal with respect to < such that $X$ is $\mathscr{P}_{k}^{(i)}$; then by Lemma 8.1(a), $X$ is nowhere $\mathscr{P}_{m}^{(j)}$ for each $\mathscr{P}_{m}^{(j)}<\mathscr{P}_{k}^{(i)}$, so $X \in \mathscr{X}_{k}^{(i)}$ by Theorem 6.25 .

Case 2: $\alpha \geqslant \omega$. Then $X$ is $\mathscr{P}_{\alpha}$. If $\alpha$ is a limit, then $X$ is $\mathscr{P}_{\alpha}$ and nowhere $\mathscr{P}_{\beta}$ for each $\beta<\alpha$, so $X \in \mathscr{X}_{\alpha}^{2}$. If $\alpha$ is a successor, then $\alpha=\beta+2$ for some $\beta<\omega_{1}$, and $X$ is $\mathscr{P}_{\beta} \cup \Sigma_{1}$ by Lemma 7.2. First suppose that $X$ is not $\mathscr{P}_{\beta} \cup$ complete; then by Lemma 8.1(a), $X$ is nowhere $\left(\mathscr{P}_{\beta} \cup\right.$ complete). So if $X$ contains no closed subset belonging to $\mathscr{X}_{\beta+1}^{2}$, then $X \in \mathscr{X}_{\alpha}^{1}$; otherwise, applying Lemma 8.1(b), we find that $X \in \mathscr{X}_{\alpha}^{2}$. If, on the other hand, $X$ is $\mathscr{P}_{\beta} \cup$ complete, then since $X$ is nowhere $\mathscr{P}_{\beta}$ by Lemma 8.1(a) and by minimality of $\alpha$, we have, in the same way as above, that $X \in \mathscr{X}_{\beta+1}^{1}$ or $X \in \mathscr{X}_{\beta+1}^{2}$.

This theorem has a number of interesting corollaries.

8.3. COROLlary. There are exactly $\omega_{1}$ homogeneous, zero-dimensional, absolute Borel sets of ambiguous class 2.

Proof. If $\alpha<\omega_{1}, i \in\{1,2\}$, then $\left|\mathscr{X}_{\alpha}^{(i)}\right|=1$ by Theorems 6.13 and 7.9, and all those spaces are topologically distinct by Theorems 6.26 and 7.25. If $X \in \mathscr{X}_{\alpha}^{(i)}$, then $X$ is of ambiguous class 2 by Theorem 3.4. Now apply Theorem 8.2 and the fact that there are only finitely many homogeneous, zero-dimensional, absolute Borel sets of class 1 .

8.4. Corollary. If $X$ is a homogeneous, zero-dimensional, absolute Borel set of ambiguous class 2, different from $C \backslash\{p\}$, and nondiscrete, then $X$ is strongly homogeneous. 
It is well known that every zero-dimensional complete space can be embedded as a closed subspace of $\mathbf{P}$, and that every zero-dimensional $\sigma$-compact space can be embedded as a closed subspace of $\mathbf{Q} \times C$.

The following theorem shows that the class of spaces characterized in this paper has a similar universal property with respect to zero-dimensional absolute Borel sets of ambiguous class 2 .

8.5. THEOREM. Let $X$ be an arbitrary zero-dimensional absolute Borel set of ambiguous class 2. Then for some limit ordinal $\alpha<\omega_{1}, X$ can be embedded as a closed subspace of the unique element $X_{\alpha}$ of $\mathscr{X}_{\alpha}^{2}$.

Proof. Since $X$ is of ambiguous class $2, X$ is $\mathscr{P}_{\beta}^{(i)}$ for some $\beta<\omega_{1}, i \in\{1,2\}$, and hence $X$ is $\mathscr{P}_{\alpha}$ for some limit ordinal $\alpha \in\left[\beta, \omega_{1}\right)$. Let $K$ be a nowhere dense copy of the Cantor set in $C$ and embed $X$ in $K$. Let $\mathscr{D}$ be a disjoint cover of $C \backslash K$ by nonempty clopen subsets of $C$ such that $\operatorname{diam}(D)<d(D, K)$ for each $D \in \mathscr{D}$. Let $X_{\alpha}$ be the unique element of $\mathscr{X}_{\alpha}^{2}$ and embed $X_{\alpha}$ as a subset $E(D)$ of $D$ for each $D \in \mathscr{D}$. Then $X$ is closed in

$$
Y=X \cup \bigcup_{D \in \mathscr{D}} E(D),
$$

and it is easily seen that $Y \approx X_{\alpha}$.

Note that there is no single zero-dimensional absolute Borel set of ambiguous class 2 which is universal in the above sense.

9. Remarks. The results of this paper suggest many questions. In the first place, since all zero-dimensional, homogeneous Borel sets of ambiguous class 2 are now characterized, we would like to have a characterization of some zero-dimensional Borel set which is of additive class 2 (i.e., a $G_{\delta \sigma}$ ), but not of multiplicative class 2 (i.e., not an $F_{\sigma \delta}$ ), or conversely. As remarked in [16], it follows from a paper of Sikorski ([24]; see also [8 and 9]) that $\mathbf{Q}^{\infty}$ is exactly of multiplicative class 2 ; to characterize this space the internal description of absolute $F_{\sigma \delta}$-sets by Sierpiński [23] might be useful.

$\mathbf{Q}^{\infty}$ is not the only homogeneous, zero-dimensional, Borel set exactly of multiplicative class 2: if $T$ is the space of Theorem $2.4(\mathrm{f})$, then $T^{\infty}$ is an $F_{\sigma \delta}$ since $T$ is, but since $T$ contains a closed copy of $\mathbf{Q}$ by Theorem 5.1(a), $T^{\infty}$ contains a closed copy of $\mathbf{Q}^{\infty}$ and hence cannot be a $G_{\delta \sigma} . T^{\infty}$ and $\mathbf{Q}^{\infty}$ are not homeomorphic, since $T^{\infty}$ contains $\mathbf{P}$ as a dense subset, whereas $\mathbf{Q}^{\infty}$ is not Baire. Hence, the following question, arises.

9.1. Question. Let $\alpha \in\left[1, \omega_{1}\right), i \in\{1,2\}$, and $X \in \mathscr{X}_{\alpha}^{(i)}$.

(a) If $X$ is Baire, is $X^{\infty} \approx T^{\infty}$ ?

(b) If $X$ is not Baire, is $X^{\infty} \approx \mathbf{Q}^{\infty}$ ?

The following question is suggested by Corollary 8.4.

9.2. Question. Does there exist a Borel subset of the Cantor set, other than $C \backslash\{p\}$ and the discrete spaces, which is homogeneous, but not strongly homogeneous?

Analyzing the results of $\$ \S 6$ and 7, one observes that the Cantor set is homogeneous with respect to dense copies of almost all homogeneous Borel sets of 
ambiguous class 2; and the only spaces which do not behave well in this respect fail to do so for a very obvious reason: when densely embedded in the Cantor set, they do not have a uniquely determined complement. Hence, we state

9.3. Question. Let $X$ be a zero-dimensional space such that if $X_{1}$ and $X_{2}$ are dense copies of $X$ in $C$, then $C \backslash X_{1} \approx C \backslash X_{2}$. Is $C$ homogeneous with respect to dense copies of $X$ ? What if $X$ is Borel? What if $X$ is (strongly) homogeneous?

Our last question is related to Theorem 4.7; one might try to substitute $\mathbf{Q}$ by some other space. It is easily seen that the theorem fails if $\mathbf{Q}$ is replaced by $\mathbf{P}$, but this is due to the fact that $C$ is not homogeneous with respect to dense copies of $\mathbf{P}$. Therefore, we have

9.4. Question. Suppose $C$ is homogeneous with respect to dense copies of $X$ and of $Y$. Is $C$ homogeneous with respect to dense copies of $X \times Y$ ? What if $X$ and/or $Y$ are Borel? What if $X$ and/or $Y$ are strongly homogeneous?

It would be particularly nice to know the answer to the above question in the case where $X$ is strongly homogeneous and $Y=C$.

ADDED IN PROOF. Characterizations of $\mathbf{Q}^{\infty}$ and $T^{\infty}$ can be deduced from a theorem of John R. Steel; the answer to Question 9.1 turns out to be yes. In a forthcoming paper, I will describe and characterize all homogeneous Borel sets in the Cantor set; from this, it can be shown that the answer to Question 9.2 is no, and the answer to Question 9.3 is yes for $X$ a homogeneous Borel set.

\section{REFERENCES}

1. P. Alexandroff and P. Urysohn, Über nulldimensionale Punktmengen, Math. Ann. 98 (1928), 89-106.

2. R. D. Anderson, On topological infinite deficiency, Michigan Math. J. 14 (1967), 365-383.

3. L. E. J. Brouwer, On the structure of perfect sets of points, Proc. Akad. Amsterdam 12 (1910), 785-794.

4. E. van Douwen, unpublished.

5. F. van Engelen, unpublished.

6. F. van Engelen and J. van Mill, Borel sets in compact spaces: some Hurewicz-type theorems, Fund. Math. 124 (1984), 271-286.

7. R. Engelking, General topology, PWN, Warszawa, 1977.

8. R. Engelking, W. Holsztyński and R. Sikorski, Some examples of Borel sets, Colloq. Math. 15 (1966), 271-274.

9. R. C. Freiwald, R. McDowell and E. F. McHugh, Jr., Borel sets of exact class, Colloq. Math. 41 (1979), 187-191.

10. A. Gutek, On extending homeomorphisms on the Cantor set, Topological Structures. II, Math. Centre Tracts, no. 115, 1979, pp. 105-116.

11. F. Hausdorff, Grundzüge der Mengenlehre, Leipzig, 1914.

12. __ Die schlichten stetigen Bilder des Nullraums, Fund. Math. 29 (1937), 151-158.

13. W. Hurewicz, Relativ perfekte Teile von Punktmengen und Mengen (A), Fund. Math. 12 (1928), 78-109.

14. B. Knaster and M. Reichbach, Notion d'homogénéité et prolongements des homéomorphies, Fund. Math. 40 (1953), 180-193.

15. K. Kuratowski, Topologie. I, 2nd ed., PWN, Warsaw, 1948.

16. J. van Mill, Characterization of some zero-dimensional separable metric spaces, Trans. Amer. Math. Soc. 264 (1981), 205-215.

17. Characterization of a certain subset of the Cantor set, Fund. Math. 118 (1983), 81-91.

18. A. V. Ostrovskir, Continuous images of the Cantor product $C \times \mathbf{Q}$ of a perfect set $C$ and the rational numbers Q, Seminar on General Topology, Moskov. Gos. Univ., Moscow, 1981, pp. 78-85. (Russian)

19. J. Pollard, On extending homeomorphisms on zero-dimensional spaces, Fund. Math. 67 (1970), 39-48. 
20. D. Ravdin, On extensions of homeomorphisms to homeomorphisms, Pacific J. Math. 37 (1971), 481-495.

21. J. Saint-Raymond, La structure Borélienne d'Effros est-elle standard?, Fund. Math. 100 (1978), 201-210.

22. W. Sierpinski, Sur une propriété topologique des ensembles dénombrables denses en soi, Fund. Math. 1 (1920), 11-16.

23. Sur une définition topologique des ensembles $F_{\text {os }}$, Fund. Math. 6 (1924), 24-29.

24. R. Sikorski, Some examples of Borel sets, Colloq. Math. 5 (1958), 170-171.

Universiteit VAN AMSTERDAM, MATHEMATISCh INSTITUUT, 1018 WB AMSTERDAM, THE NeTHERLANDS 